\title{
Jean BLOUIN
}

journaliste

(1982)

\section{GÉRARD BERGERON. de l'autre côté de l'action.}

Un document produit en version numérique par Réjeanne Toussaint, ouvrière bénévole, Chomedey, Ville Laval, Québec

Page web. Courriel: rtoussaint@aei.ca

Dans le cadre de la collection: "Les classiques des sciences sociales"

Site web: http://www.uqac.ca/Classiques_des_sciences_sociales/

Une collection développée en collaboration avec la Bibliothèque

Paul-Émile-Boulet de l'Université du Québec à Chicoutimi

Site web: http://bibliotheque.uqac.uquebec.ca/index.htm 


\section{Politique d'utilisation de la bibliothèque des Classiques}

Toute reproduction et rediffusion de nos fichiers est interdite, même avec la mention de leur provenance, sans l'autorisation formelle, écrite, du fondateur des Classiques des sciences sociales, Jean-Marie Tremblay, sociologue.

Les fichiers des Classiques des sciences sociales ne peuvent sans autorisation formelle:

- être hébergés (en fichier ou page web, en totalité ou en partie) sur un serveur autre que celui des Classiques.

- servir de base de travail à un autre fichier modifié ensuite par tout autre moyen (couleur, police, mise en page, extraits, support, etc...),

Les fichiers (.html, .doc, .pdf, .rtf, .jpg, .gif) disponibles sur le site Les Classiques des sciences sociales sont la propriété des Classiques des sciences sociales, un organisme à but non lucratif composé exclusivement de bénévoles.

Ils sont disponibles pour une utilisation intellectuelle et personnelle et, en aucun cas, commerciale. Toute utilisation à des fins commerciales des fichiers sur ce site est strictement interdite et toute rediffusion est également strictement interdite.

L'accès à notre travail est libre et gratuit à tous les utilisateurs. C'est notre mission.

Jean-Marie Tremblay, sociologue

Fondateur et Président-directeur général, LES CLASSIQUES DES SCIENCES SOCIALES. 
Cette édition électronique a été réalisée par Réjeanne Toussaint, bénévole, Courriel: rtoussaint@aei.ca

Jean Blouin

\section{GÉRARD BERGERON. de l'autre côté de l'action.}

Montréal: Éditions Nouvelle Optique, 1982, 232 pp. Collection : Traces et paroles.

[Autorisation formelle accordée, le 12 avril 2005, par Mme Suzanne PatryBergeron, épouse de feu M. Gérard Bergeron, propriétaire des droits d'auteur des œuvres de M. Gérard Bergeron]

Polices de caractères utilisée :

Pour le texte: Times New Roman, 12 points.

Pour les citations : Times New Roman, 12 points.

Pour les notes de bas de page : Times New Roman, 12 points.

Édition électronique réalisée avec le traitement de textes Microsoft Word 2008 pour Macintosh.

Mise en page sur papier format : LETTRE (US letter), 8.5'” $\mathrm{x}$ 11')

Édition numérique réalisée le 6 juillet 2010 à Chicoutimi, Ville de Saguenay, province de Québec, Canada. 


\section{Jean BLOUIN (1982)}

GÉRARD BERGERON de l'autre côté de l'action

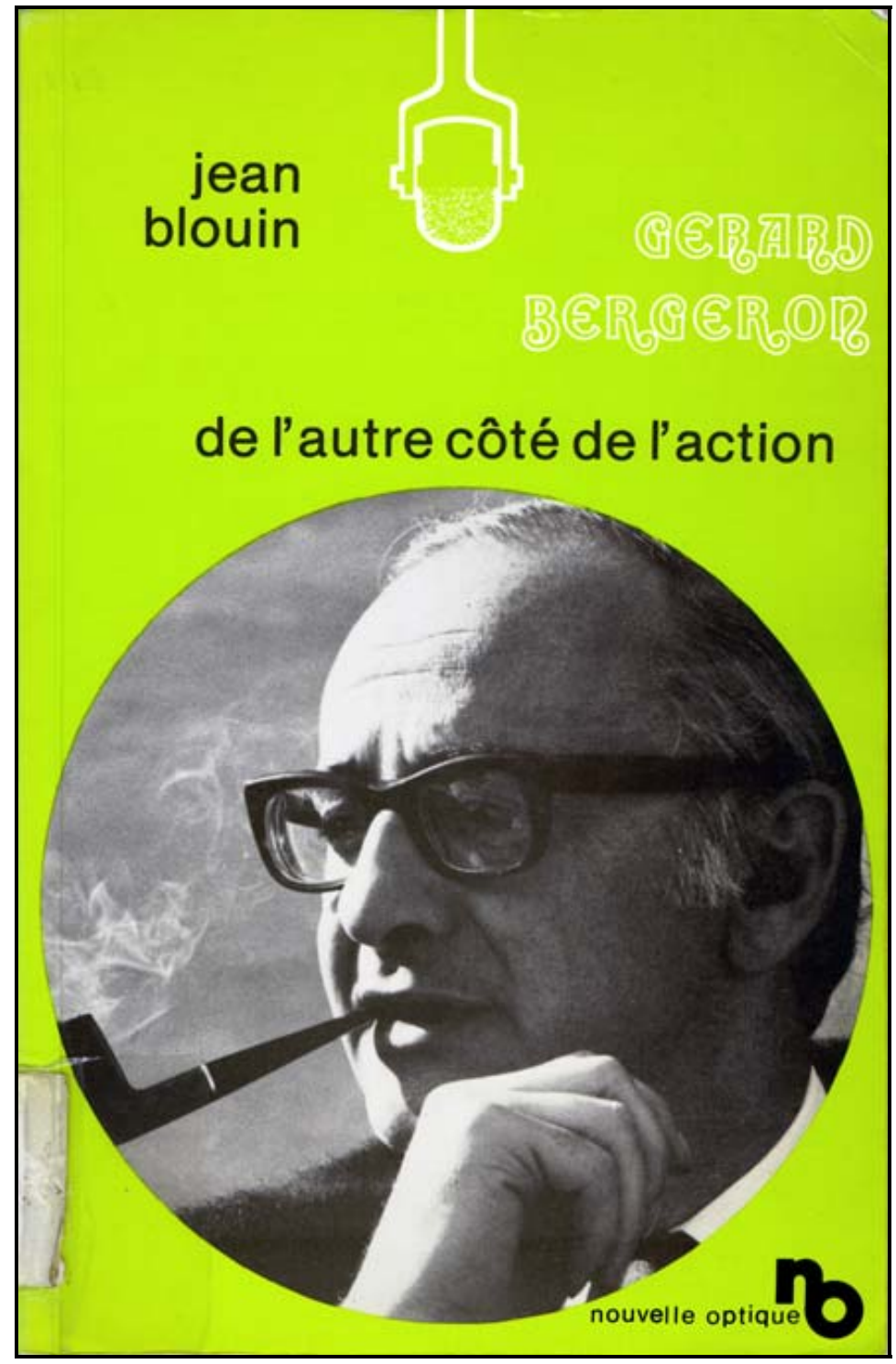

Montréal: Éditions Nouvelle Optique, 1982, 232 pp. Collection : Traces et paroles. 


\section{Table des matières}

Quatrième de couverture

1. Prologue par Jean Blouin

2. $\quad$ Premier entretien : le citoyen

«... le besoin de comprendre »

3. Deuxième entretien : l'universitaire

« ... naturellement, en toute nécessité »

4. Troisième entretien : le théoricien

«... par une espèce d'instinct de conservation »

5. Quatrième entretien : le journaliste

« ... de l'autre côté de l'action »

6. Cinquième entretien : l'essayiste

«... l'État, toujours Lui »

Document. L'homo quebecensis

7. Sixième entretien : génération et environnement

«... l'idéal d'une philosophie hédoniste du travail »

8. $\quad$ Petite géographie personnelle

New York, Paris et Berthier-sur-Mer

- le corps et l'âme de New York (août 1946)

- paris, capitale des métropoles (mai 1951)

- à Berthier-sur-mer où commence «la mer toujours recommencée» (août 1981)

9. Épilogue par Gérard Bergeron

Notice biographique

Bibliographie 
GÉRARD BERGERON.

de l'autre côté de l'action.

\section{QUATRIÈME DE COUVERTURE}

$\underline{\text { Retour à la table des matières }}$

Nos vedettes s'adonnent au sport ou à la politique. Les premiers n'ont pas comme vocation première de penser ; la pensée des seconds est univoque, simplificatrice, comme si l'exigence de l'action partisane réduisait l'homme à une rengaine. Pour l'intelligence du monde et de notre milieu, il faut aller voir de l'autre côté de l'action, qui n'est pas tant son contraire que son complément, ouverture à l'histoire, à tous les possibles, refus des idéologies toutes faites, fût-ce pour la bonne cause. Il n'y a pas foule. Mais vous rencontrerez à coup sûr Gérard Bergeron, dont on ne sait pas s'il en est l'archétype ou le dernier des Mohicans.

Concentrant en lui tous les ingrédients de la partisannerie (compétence de politologue, passion pour la chose publique, interventions journalistiques répétées, connaissance des hommes de pouvoir), comment a-t-il pu, dans le débat manichéen des 25 dernières années, résister à tous les rangs d'oignons qui se sont offerts à lui : l'opposition libérale de Lapalme-Lesage, l'équipe du tonnerre de la Révolution tranquille, le « French power » et les Trois Colombes, le mouvement indépendantiste qui a abouti au gouvernement Lévesque ? Et cela, après avoir invité bon nombre des têtes d'affiche de ces différentes équipes à faire le saut dans l'action?

Poser la question, c'est déjà avoir un pied dans l'univers mental de Gérard Bergeron. Au fil des entretiens, vous découvrirez un être complexe, irréductible, foncièrement binaire : à la fois de la tour d'ivoire et de la place publique, d'ici et 
d'ailleurs, nationaliste et internationaliste, individualiste et communautariste, sensible et rationnel. Ce nœud de contradictions apparentes constituent son essence même, au sens philosophique comme dans son acception " énergétique ». Non seulement ses contradictions le maintiennent-elles en un équilibre toujours à refaire, tel un funambule, mais elles, seules, lui permettent d'avancer et de rechercher un progrès qui ne soit pas la négation d'un pan de la réalité ou qui se fasse à son détriment. 
[9]

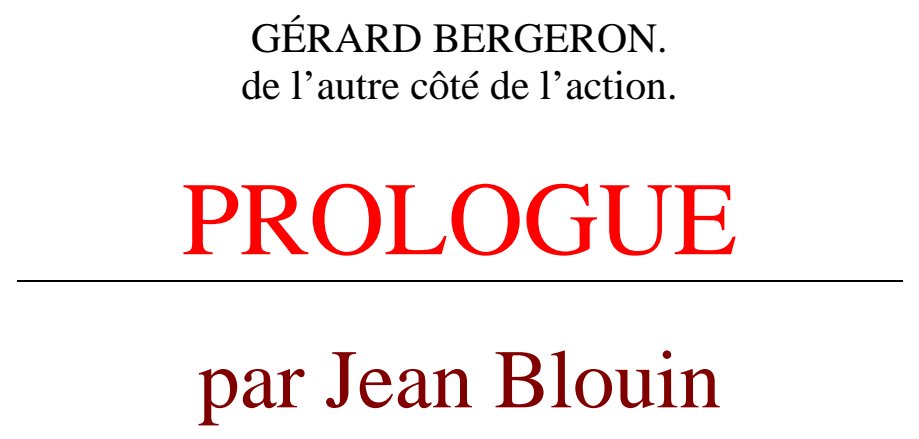

Retour à la table des matières

Nos vedettes s'adonnent au sport ou à la politique. Les premiers n'ont pas comme vocation première de penser ; la pensée des seconds est univoque, simplificatrice, comme si l'exigence de l'action partisane réduisait l'homme à une rengaine. Pour l'intelligence du monde et de notre milieu, il faut aller voir de l'autre côté de l'action, qui n'est pas tant son contraire que son complément, ouverture à l'histoire, à tous les possibles, refus des idéologies toutes faites, fût-ce pour la bonne cause. Il n'y a pas foule. Mais vous rencontrerez à coup sûr Gérard Bergeron, dont on ne sait pas s'il en est l'archétype ou le dernier des Mohicans.

Concentrant en lui tous les ingrédients de la partisannerie (compétence de politologue, passion pour la chose publique, interventions journalistiques répétées, connaissance des hommes de pouvoir), comment a-t-il pu, dans le débat manichéen des 25 dernières années, résister à tous les rangs d'oignons qui se sont offerts à lui : l'opposition libérale de Lapalme-Lesage, l'équipe du tonnerre de la Révolution tranquille, le "French power » et les Trois Colombes, le mouvement indépendantiste qui a abouti au gouvernement Lévesque ? Et cela, après avoir invité bon nombre des têtes d'affiche de ces différentes équipes à faire le saut dans l'action? 
[10] Poser la question, c'est déjà avoir un pied dans l'univers mental de Gérard Bergeron. Au fil des entretiens, vous découvrirez un être complexe, irréductible, foncièrement binaire : à la fois de la tour d'ivoire et de la place publique, d'ici et d'ailleurs, nationaliste et internationaliste, individualiste et communautariste, sensible et rationnel. Ce nœud de contradictions apparentes constituent son essence même, au sens philosophique comme dans son acception « énergétique ». Non seulement ses contradictions le maintiennent-elles en un équilibre toujours à refaire, tel un funambule, mais elles, seules, lui permettent d'avancer et de rechercher un progrès qui ne soit pas la négation d'un pan de la réalité ou qui se fasse à son détriment.

Ce livre-entretiens nous fait revivre l'itinéraire signifiant de l'un des intellectuels québécois les plus importants de l'après-guerre. Un des pères fondateurs des sciences sociales dans notre milieu, universitaire réputé depuis plus de trente ans, essayiste, chroniqueur politique bien connu des lecteurs du Devoir depuis 1956, alors qu'il signa du pseudonyme d'Isocrate une analyse percutante du régime duplessiste à peine reporté au pouvoir, Gérard Bergeron est aussi - et surtout, quant à lui - un théoricien de la science politique. Sa réputation, à ce chapitre, dépasse largement les frontières du Québec, Raymond Aron, préfacier de son premier ouvrage de théorie politique, Fonctionnement de l'État, avait été frappé par la synthèse des courants européen et américain de la science et de la culture qu'il retrouvait chez le politologue de l'université Laval. Cet enracinement à plusieurs niveaux place Gérard Bergeron parmi les grands sociologues politiques qui, parallèlement à leurs recherches en tour d'ivoire, s'intéressent activement aux problèmes de la Cité.

À quelle enseigne philosophique, idéologique et psychologique loge-t-il ? Comment le théoricien, l'essayiste et le journaliste tiennent-ils ensemble en la personnalité unique de l'homme-citoyen ? En somme, une série de considérations et de confidences qu'on ne livre pas autrement que par la provocation de l'entretien prolongé.

$$
* * *
$$

Pour l'intelligence du livre, je me dois de préciser l'espèce [11] de pacte conclu à l'origine de nos entretiens. Gérard Bergeron s'est engagé à répondre à 
toutes les questions, y compris les questions provocantes ou les questions-pièges (qu'il savait d'ailleurs discerner). C'était ma condition préalable. Il a joué la règle du jeu. Le lecteur constatera que je n'ai pas été complaisant.

En contrepartie - sa condition préalable - nous n'avons pas abordé ce qu'il appelle son « domaine réservé » : sa vie intime, personnelle, familiale qui lui appartient et qui n'est pas d'intérêt public, affirme-t-il avec conviction. Il s'est contenté de dire qu'il mène une vie de famille heureuse avec ses enfants « tous beaux et intelligents » (dont les intérêts vont de la sociologie de la science à la mécanique automobile en passant par la médecine et la musique ancienne) : Alain, Annie, Sylvain et Martin, et de son épouse, Suzanne Patry, une femme remarquable et qui poursuit une carrière distinguée de clinicienne en psychopédagogie. Il s'est, de même, refusé à raconter son enfance et son adolescence. La narration débute avec l'entrée à l'université. Je renvoie le lecteur à la bibliographie et à la notice biographique à la fin du volume pour en savoir davantage. Nous avons ainsi fait l'économie d'un chapitre.

Dernière exigence de Gérard Bergeron : une iconographie réduite à sa plus simple expression. Ce que le livre perd « en potinage et en image », il le gagne peut-être en substance du message. Nous avons par ailleurs, le directeur de la Nouvelle Optique, Hérard Jaclotte, et moi « négocié » la publication de trois documents qui complètent à merveille, nous a-t-il semblé, le portrait de Bergeron. Ces documents, réunis à la fin du volume, forment une «petite géographie personnelle » aussi intéressante qu'inédite.

Quelques mots sur le mode de fabrication comme tel. Après quelques séances préliminaires en juillet et en août, à Québec, l'essentiel du travail s'est fait au chalet de Gérard Bergeron à Berthier-sur-Mer, où nous nous sommes retirés du 18 au 24 août. Chaque matin, nous fixions les grandes lignes de l'interview de la journée, généralement un chapitre. L'enregistrement s'effectuait en deux étapes de deux à trois heures chacune. Cette planification n'a pas empêché les entretiens d'emprunter souvent des tangentes imprévues. Ma pratique journalistique m'a enseigné à [12] attendre, et à exploiter aussitôt, la moindre brèche ouverte par l'interlocuteur.. La révision respecte le caractère spontané, à bâtons rompus, de certains passages. Nous avons mis le point final au travail de révision à mon domicile d'Outremont, dans la première quinzaine de novembre. 
$\mathrm{Au}$ terme de cette expérience, permettez deux annotations personnelles. J'ai découvert en Gérard Bergeron un homme d'une culture vaste et d'une curiosité sans limites. Par la magie du dialogue, il a trouvé plaisir à raconter comment son oeuvre d'apparence disparate s'est faite et continue de se faire. Nos tours d'horizon matinaux débordaient la matière de ce livre et m'ont beaucoup appris sur «les idées, les événements et les hommes » contemporains. Mon mérite est de l'avoir amené à se confier à fond, à l'intérieur des balises indiquées plus haut, sans fausse modestie ni esquives.

Je me permets, enfin, de remercier Marie-Josée, mon épouse. Non seulement a-t-elle été la première lectrice, attentive et combien critique, du manuscrit, mais elle en a aussi dactylographié de longs extraits, au détriment de ses propres travaux d'étudiante. Sans elle, je n'aurais pas réussi l'imprudent pari de faire paraître ce livre au début de 1982.
\end{abstract}

Jean Blouin

Outremont, le 3 décembre 1981 
[13]

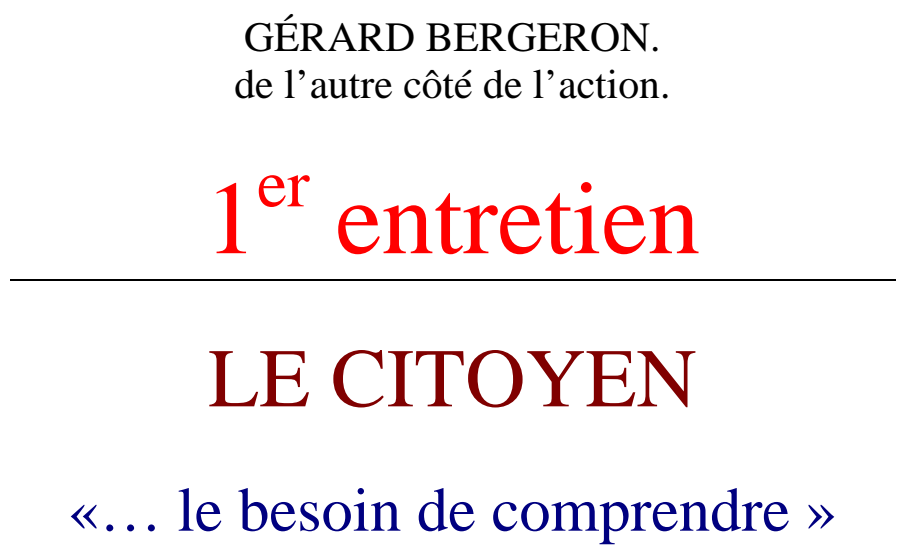

$\underline{\text { Retour à la table des matières }}$ 
J.B. Réagissant à un article très dur que vous aviez écrit sur les maux, travers et difficultés du fédéralisme canadien - allant jusqu'à traiter notre Confédération de " vieille dame indigne »selon le titre d'un film célèbre -, le président du Parti québécois d'alors, titulaire d'une rubrique dans un journal, vous enjoignait à être conséquent avec votre diagnostic et à vous « brancher "une fois pour toutes. Votre réplique parut dans Le magazine Maclean de septembre 1971, où vous aviez vous-même une collaboration régulière. Elle portait sur la notion de " branchisme ». C'est assez étonnant qu'un politologue se fasse théoricien du branchisme par une défense du nonbranchisme personnel !

G.B. Pas du tout si vous n'oubliez pas que mon article était une réponse à une interpellation directe qu'un chef de parti me faisait. Le branchisme s'entendait donc comme une adhésion partisane comme je me faisais critique sévère d'un état de choses constitutionnel, qui était ce contre quoi le parti en question s'était fondé. Je répondais du point de vue de ma position d'analyste des choses politiques, revendiquant le droit de le faire hors de l'adhésion partisane précisément. De ce point de vue, et de ce seul point de vue, je défendais mon non-branchisme personnel dans l'accomplissement d'un rôle personnel - sur lequel vous vous proposez de m'interroger à fond pendant ces entretiens.

J.B. Vous ne faisiez donc pas du non-branchisme une prescription ou un conseil pour les autres?

G.B. Absolument pas. Cela était explicitement dit dans le texte en question. Surtout que le branchisme, disons positif, est l'une de mes idées directrices. C'est même sous ce thème - sans le pittoresque de l'expression - que j'ai fait mes débuts dans le journalisme de collaboration externe il y a vingt-cinq ans. Je disais aux gens de ma génération, à la fin du règne de Duplessis, qu'il était temps pour ceux d'entre nous, taillés pour l'action politique et impatients d'en faire, de se décider à prendre leurs risques en s'engageant dans un parti politique qui était à prendre 
littéralement afin de nous faire sortir de cet ancien régime interminable. Et la suite des événements allait montrer que l'analyse de situation que je faisais alors était fondée...

\section{J.B. ...ce qui allait nous valoir nos deux premiers ministres !}

G.B. Mais plus tard. C'était en 1956, 1957 que je prônais ce branchisme-là. L'adhésion de René Lévesque au parti libéral du Québec allait se produire en 1960 ; et cinq ans plus tard, celle de Pierre Elliott Trudeau au parti libéral du Canada. Je m'empresse d'ajouter deux précisions. D'abord ce n'est pas à ces deux-là seuls que je m'adressais, mais à d'autres personnalités, marquantes également, dont certaines feront aussi le bond pour constituer plus tard les noyaux durs des équipes de la Révolution tranquille à Québec et du French power à Ottawa. L'autre précision est que je n'ai pas eu de succès à l'époque. Il n'y avait guère que Jean Marchand pour être déjà convaincu d'avance, et qui se préparait à se tenir en disponibilité politique, hésitant du reste assez longtemps entre les scènes provinciale ou fédérale. Ce n'est donc pas sous l'influence directe de mes papiers de l'époque que nos grands leaders allaient plonger plus tard. Il y avait eu, dans l'intervalle, la mort de Duplessis, la grève de Radio-Canada, le nouveau leadership de Lesage ; et puis, dans le cas de Trudeau, la montée d'un nouveau [17] nationalisme au Québec au début des années 1960. Le branchisme de ces deux-là et des autres se serait produit de toutes façons. Mais le point à marquer c'est que, positivement et avant tout le monde, j'aurai été « théoricien du branchisme » comme vous dites. Beaucoup plus tard, dans la foulée du 15 novembre 1976, je suis revenu fréquemment sur ce thème. Mais il s'agissait alors d'un branchisme collectif, celui du peuple du Québec qui devait avoir enfin l'occasion de se brancher sur les grandes questions du fédéralisme (à maintenir) ou de l'indépendance (à faire). Avec des effets encore confus au point où nous en sommes actuellement (en août 1981).

J B. Être non partisan peut signifier être de nulle part. N'est-on pas toujours branché finalement? 
G.B. Dans mon texte de 1971 que vous évoquiez au début, je disais que ce sont les branchés qui font marcher les sociétés et, j'ai presque envie d'ajouter qu'ils les font d'autant plus marcher qu'ils sont solidement branchés ! Je réclame seulement le droit d'entendre mon type d'engagement civique en dehors du militantisme ou même de l'adhésion partisane. Quand je vote à titre de citoyen - individu comme tout le monde, je me branche : mon vote ne se fractionne pas, ne se nuance pas. J'ai toujours voté, ne me suis jamais abstenu. Vous dire que c'est avec beaucoup d'enthousiasme dans la plupart des cas, non! Comme tous ceux qui n'ont pas l'adhésion facile - non pas par indifférence mais par exigence - je vote fréquemment pour le moindre mal, le risque le moins mauvais. Je tiens au secret de l'isoloir. Les positions que j'y prends ne concernent que moi devant ma conscience civique. Je ne vois absolument pas qu'elles soient d'intérêt public. C'est hautain ou modeste, vous avez le choix de l'interprétation : moi, je suis à l'aise dans la mienne.

En outre, je ne sers d'aucune caution à qui que ce soit ; je ne suis pas marqué de l'étiquette limitatrice de l' « adversaire »(dont les points de vue sont disqualifiés à ce titre par la moitié de votre public potentiel ). Et surtout la plus ou moins grande validité, ou éventuelle [18] utilité civique, des papiers ou études que je publie n'a pas de rapport direct ni nécessaire avec mes propres choix d'individucitoyen. Mais comme je parle dru assez souvent, on voit quelles sont mes préférences du moment ou sur telle question. J'espère tout de même me faire comprendre bien que n'employant pas le mode unilatéral du partisan.

\section{J. B. Vous répondez selon le vieux dilemme de l'analyste qui se doit d'être le plus objectif possible et du citoyen qui n'a pas à répudier sa propre subjec-} tivité.

G. B. Je répète que ma position n'implique pas d'hésitation particulière pour le citoyen quant à ses choix. Mais les rapports de la vie privée, et même de la vie intime, sont nombreux et subtils. Je ne gagnerais rien, j'aurais plutôt tout à perdre à céder à mes humeurs, à projeter sur la place publique des états d'âme personnels, et même à y régler (sans l'avouer) mes problèmes intérieurs par des adhésions à des causes qui, parce que collectives, dépassent nos petites personnes. Il 
reste évidemment un certain élément de mystère sur mes choix profonds. Mais si je peux encore parler aux fédéralistes et aux indépendantistes avec, je crois, une certaine présomption de crédibilité, c'est qu'ils ne m'appliquent pas le sceau du partisan ou de l'adversaire, qui me disqualifierait à priori. Vous avouerais-je un petit secret ? Comme il m'arrive parfois d'avoir des feed-back de mes écrits, ce sont « les meilleurs », parmi les clans qui s'opposent, qui acceptent volontiers le bien-fondé de cette position particulière que je prétends maintenir.

\section{J. B. Cette crédibilité des deux bords ne vous oblige-t-elle pas à mettre vo-} tre sensibilité, à fortiori votre passion, sur la glace? L'analyste y trouve son intérêt. Le citoyen, lui ?

G. B. Vous avez parlé de « passion ». Oui, je suis un passionné de la politique, des problèmes de la Cité. Mais pour [19] les comprendre. Par besoin de comprendre. De voir clair dans la bousculade et le fouillis. Il faut être loin des pouvoirs pour les voir. Et non pas « dedans », ou tout près. Je pourrais élaborer longtemps sur ce paradoxe apparent. Non, je ne me sens pas aliéné dans ma vie d'intellectuel ni de citoyen. Ce qui m'aliénerait, ce serait précisément le mode de communication unilatéral auquel je devrais consentir pour des fins, disons, de solidarité envers des co-partisans et des militants. Je ne me sens pas d'inclination à devenir un des soldats des grandes armées qui s'affrontent qui, d'ailleurs, ne manquent pas d'effectifs ! En outre, je suis convaincu que je serais d'une faible utilité dans ce rôle de fantassin, et même de souffleur des répliques ou de rationnalisateur des politiques derrière les états-majors.

\section{J. B. Vous avez toujours été comme ça ?}

G. B. 'Très jeune, en observant comment s'opéraient les rapports d'autorité et de pouvoir (parents - enfants ; dirigeants de cercles ou d'équipes - membres ; présidents de classe - condisciples ; ou simplement vie interne des gangs, etc.), j'ai compris ! J'ai compris la base du jeu de la politique. J'ai surtout compris que j'y serais malhabile, voire inapte. C'était me vouer à une certaine solitude, mais vo- 
lontairement consentie. Plus tard, graduellement je comprendrai que cette attitude, non seulement pourrait fonder ce qu'on appelle communément une crédibilité ou même une pureté d'intention, mais qu'elle était une espèce de conditionnement technique du travail que j'aurais à faire en ce domaine. Si je prétends mieux voir la politique que ceux qui la font ou tentent de la faire, il ne faut tout de même pas que je me mette dans les mêmes conditions qu'eux, c'est-à-dire le « nez dedans »!

\section{J. B. C'est quand même s'interdire une action directe, qui peut être assez séduisante.}

G. B. Encore une fois ce ne sont pas les gens de cette espèce qui font fonctionner les sociétés, qui sont au principe des changements. Il n'est pas grave qu'il y en ait quelques-uns de cette espèce, s'ils sont faits pour ça. L'autre « espèce » est assez bien peuplée, merci !

\section{J. B. N'empêche que le chroniqueur politique Bergeron agace, met sou-} vent en rogne les branchés. Ce n'est pas la meilleure façon de se faire des amis !

G. B. Je pourrais aussi retourner la question en répondant : de ne pas trop se faire d'ennemis, non plus ! Mais revenons au sérieux Cette attitude, quand je l'explique, me vaut parfois des témoignages d'estime inattendus, tel celui d'un de nos tout premiers écrivains, se déclarant lui-même tout ce qu'il y a de plus branché (et dont je vous ai montré la lettre tout à l'heure). Je suis tout à fait à l'opposé de vous, me dit-il, en substance, mais je vous trouve cohérent et non destructeur de l'action, comme tel autre intellectuel qu'il connaît bien et qu'il trouve perdu en politique. Quand je suis mis en cause sur le caractère d'étrangeté de ma position comme vous le faites actuellement en me mettant sur la sellette - je ne m'enferme pas dans un silence hautain ou méprisant. Je riposte par des textes courts et explicites comme j'essaie de le faire par mes réponses. La rareté de la fonction que j'essaie d'assumer - parce qu'elle me convient et qu'elle est, je pense, défendable vaut bien qu'on l'explique à l'occasion. 
Cette liberté extraordinaire dont je dispose, et que je m'impose, fait aussi que ma critique n'a pas l'inélégance de celle des co-combattants qui se tirent dans les jambes ! La critique libre est toujours difficile à l'intérieur de la solidarité partisane. Voyez en Europe l'inconfort pénible des intellectuels dans les partis idéologiques : ils cèdent, s'homogénisent, sont chassés ou évacuent la place. Voyez ici au Canada, l'ancien groupe Waffle qui a été digéré par le NPD. Voyez encore les indépendantistes irréductibles à l'intérieur du PQ vis-à-vis de la ligne étapiste du parti : ils ne peuvent s'y opposer que dans la mesure d'une fidélité [21] sans cesse à réaffirmer à l'équipe dirigeante. N'étant pas inféodé à un parti ou à une faction, je n'ai pas à rompre publiquement ou à proclamer une dissidence pour gagner le droit de dire ce qui ne me convient pas.

\section{J. B. Nous en venons graduellement à la grande question de l'indépen-} dance du Québec. Il y a bien des gens qui souhaiteraient vous voir adopter des prises de position plus fermes, moins ambivalentes.

G. B. C'est que la situation est objectivement ambivalente, pour ne pas dire carrément ambiguë... C'est le temps d'évoquer le mot de l'historien Maurice Séguin qui, dans une conférence célèbre, il y a une vingtaine d'années ${ }^{1}$, s'interrogeait sur «notre sort de nation annexée, la mieux entretenue de monde » et concluait que « nos maîtres, les Anglais ne seraient pas dignes d'avoir été nos maîtres pendant deux siècles s'ils se laissaient démolir facilement ». Tout y est en ces quelques lignes. Nous sommes une «nation annexée » : donc, dont le devoir premier est de se désannexer, de faire son indépendance enfin ! C'est la vocation à l'indépendance. L'idée en a couru, plutôt timide ou nostalgique, depuis une couple de siècles, puis en force croissante depuis une vingtaine d'années. En outre, ce qui n'était pas le propos de Séguin, cette « nation annexée » est en possession d'un État, de l'État fédéré d'un État fédéral : puis vient tout le reste, étendue et richesse du territoire, population forte et développée, culture dominante homogène et capable de se développer. Se trouvait-il parmi la centaine des nouveaux États indépendants depuis la guerre plus de cinq ou six dont la société pouvait présenter des

1 Maurice Séguin, L'idée d'indépendance au Québec, Montréal, Les éditions Boréal-Express, 1977, p. 65, 66, 67. 
titres aussi clairs à l'indépendance et à la souveraineté internationale que la nôtre ? Cela qui devrait être tenu pour une évidence, et que j'ai rappelé dans mes écrits plutôt dix fois qu'une, n'est pas le problème. Le problème est...

\section{J. B. ... que nous ayons été « la nation la mieux entretenue du monde " comme disait Maurice Séguin?}

G. B. Oui, et que nous le croyons encore malgré le malfonctionnement chronique du fédéralisme dont nous sommes affligés. Le point ici est que comme société qui, malgré tout, n'a pas été trop malmenée par l'histoire, nous ne nous sentions pas abîmés suffisamment pour prendre des risques fous. D'où notre conservatisme profond, notre esprit de Normand calculateur devant le mur des incertitudes tout au moins, sinon en plein conscience de périls graves dont certaine propagande nous abreuvait à l'époque du référendum. Nous voudrions l'indépendance, mais nous ne la voulons pas. C'est cela.

\section{J.B. Et « les Anglais » ne laisseraient pas faire ?}

G. B. Ils ne sont plus « nos maîtres » au sens où la rétrospective de Séguin les qualifiait dans l'histoire des deux derniers siècles. Ils sont à tout le moins, là, dans dix capitales dont une centrale, les plus gros, les plus forts, les plus riches, les plus nombreux. C'est en sécession d'eux, et sans aide efficace de l'extérieur, qu'il faudrait arracher l'indépendance d'une façon ou de l'autre. Et comme il est hors de propos de faire l'indépendance autrement que par des moyens démocratiques, voyez le double objectif qui est doublement énorme : rallier une majorité, suffisamment forte pour être incontestable, de voix favorables à l'indépendance pour avoir chance de faire basculer l'extraordinaire force de résistance du reste du Canada.

\section{J. B. Et cela vous ne voyez comment cela pourrait se produire?}


G. B. Non. Et je vois de façon beaucoup plus claire comment les facteurs défavorables à l'indépendance (et en premier lieu, notre « ambivalence », qui est une situation congénital) ne pourront être surmontés. Il se peut que je vois mal. J'accepte de me tromper. Mais la conclusion de ce qui précède, c'est que l'indépendance, toute désirable, toute naturelle et inscrite dans [23] la nature des choses, n'apparaît pas faisable dans les circonstances prévisibles. Comment voulez-vous que le citoyen en question aborde le problème autrement que par cette constatation ? Elle n'a rien d'exaltant et exclut tout emballement.

\section{J. B. Mais ceux qui croient à l'indépendance}

G. B. Justement, c'est une croyance donc, qui se situe d'emblée hors, sinon contre, les amorces de l'esprit critique. Il y a aussi les croyants de l'autre secte, qui célèbrent le culte du Canada et du fédéralisme qu'ils font s'équivaloir. Ce n'est pas aisé de s'adresser en même temps aux croyants de deux cultes qui s'estiment mutuellement impies. C'est ce que j'appelais, dans mes cogitations jusqu'à maintenant secrètes, l'impossible dialogue entre « les deux abominations » : celle de l'aberration d'un côté, celle de la perversion de l'autre.

Pour le croyant du fédéralisme, l'indépendance du Québec est un acte malfaisant, un charcutage du Canada, un cataclysme. « Un crime contre l'histoire de l'humanité », pour reprendre les mots de l'actuel premier ministre du Canada. Pour notre fédéraliste inamovible, c'est une perversion que de penser autre chose. Quant aux motifs derrière cette recherche de l'indépendance, ils ne peuvent qu'être bas, petits, mesquins. Pour le croyant de l'indépendance, c'est tout au contraire une chose toute naturelle, c'est même le devoir premier et sacré de notre fraternité, un impératif de notre dignité devant l'Histoire que d'aller au bout de ce processus de libération progressive. Cette indépendance aurait dû être faite bien auparavant, elle est inscrite dans notre destin, nous en possédons tous les éléments constitutifs, nous avons les moyens de la réaliser, y compris les moyens techniques et économiques, etc. Bref, il est aberrant de penser autre chose, d'accepter un destin moindre. Ces deux raisonnements «à l'abomination » sont chacun d'eux d'une stricte logique. J'en ai sérié les arguments valables et très peu variables selon les circons- 
tances. Il y a de quoi alimenter les débats et les [24] énergies politiques des six prochaines générations.

J. B. Vous adopteriez plutôt un point de vue d'agnostique entre les adhérents des deux religions politiques?

G. B. D'agnostique qui pose la question hors des théologies de ces deux religions de salut. Nous serions plusieurs dans cette troisième catégorie ! Et pas seulement d'« indécis »... selon l'expression consacrée des sondages. Aux deux pôles des opinions, fédéralistes inamovibles et indépendantistes irréversibles, se forment des minorités assez fortes, mais ne dépassant pas les 20\% pour chacune d'elles. Entre ces deux minorités s'étale la forte majorité de $60 \%$, faite aussi bien d'agnostiques inquiets que d'indifférents jusqu'à l'inertie. Ce gros centre réunit tous ceux qui, pour quelque raison, ne croient pas aux vertus immanentes de l'une ou l'autre des formules avancées. Il faut que s'élèvent, de ce gros «tiers », des voix qui interrogent les théologiens fulminants des deux autres petits «tiers ». Disons que je suis une de ces voix-là, agnostique, ou plutôt sceptique devant des objectifs élevés à une hauteur de positivité absolue.

\section{J. B. Mais l'agnostique que vous êtes a tout de même avoué tout à l'heure} qu'il pouvait se tromper. Et je crois deviner dans vos propos que les valeurs de progrès seraient plutôt du côté de l'indépendance.

G.B. Mon inquiétude fondamentale n'est pas que l'indépendance ait lieu ou pas, mais qu'elle ne rate pas si on s'y engage réellement ou qu'elle en vienne à s'imposer vraiment un jour. Parce qu'une « révolution » de cet ordre qui serait ratée serait un très lourd manteau de plomb qu'on aurait à traîner pendant très longtemps. Ce serait bien plus grave à porter que les complexes de l'actuelle dépendance. Même les révolutions réussies dont on se gargarise devant l'histoire ne sont pas toujours belles à vivre dans le concret quotidien. Ça meuble les places publiques de monuments pour [25] la contemplation des fils des révolutionnaires mais qui en sont rarement dignes. Aussi, ce n'est pas le quoi ni le pourquoi de 
l'indépendance qui m'occupe l'esprit - j'en rajouterai plutôt à l'argumentation qu'on nous sert à ce propos - mais le comment elle pourrait être possible. L'indépendance ne devient une chose sérieuse que si le projet en apparaît faisable.

\section{J. B. On a déjà entendu parler d'autres voix inquiètes à ce sujet : un Jac-} ques Grand'Maison, un Jean-Paul Desbiens, par exemple, se demandent si nous aurions les moyens d'en payer les coûts.

G. B. Une fois embarqués, nous trouverions bien les moyens d'en « payer » le prix. Mon inquiétude est antérieure à celle-là : avons-nous simplement les moyens de nous engager dans un risque de cette ampleur? Quand nous parlions de «branchisme » tout à l'heure, il ne s'agissait pas que de celui d'individus, mais aussi de la collectivité. Or, que veut le peuple ? Ce que les dernières consultations populaires électorales et référendaires ont établi et que confirment les tendances de sondages à la centaine : ne pas avoir à décider une rupture totale, mais profiter plutôt des circonstances et des rapports de force. S'il s'agit d'endosser un bon programme ministériel ou de juger une performance gouvernementale, nous sommes bien prêts à accorder sa chance au Parti québécois. Mais s'il nous pose la question portant sur le principe du régime, fût-ce en termes doublement rassurants, nous ne lui accordons pas le feu vert. Il ne m'apparaît pas, à aucun signe ou indice actuellement visible, que ce peuple, qui s'est toujours conduit ainsi depuis toujours, puisse changer d'attitude sur l'envergure du risque. Il ne semble pas qu'il puisse dégager cette dynamique interne très forte qui lui permette d'en imposer aux très forts pouvoirs d'en face (la capitale fédérale), de chaque côté (l'Ontario et les autres régions) et d'en dessous (le plus puissant État du monde à tous égards). Sans cette espèce de pression interne très forte, sans cette espèce d'implosion avant l'explosion, l'indépendance n'apparaît pas faisable. Elle n'aura pas lieu.

J. B. Mais peut-être fallait-il faire comme si, au contraire, elle était faisable ? 
G. B. Oui, pour plusieurs raisons. La première étant que ce débat, renvoyé de génération en génération, nous le tenions enfin. Et qu'il aboutisse à quelque chose, fût-ce à un constat de notre relative détermination, à cause de notre relative puissance. Il y a aussi que c'est notre contribution à la reconversion d'un régime assez vermoulu et qu'il faudra refaire de toute façon un jour ou l'autre. C'est embêtant de parler ainsi à ceux des nôtres pour lesquels l'accession à l'indépendance est un projet de dignité collective et de nécessité historique. Mes propos volent bas en comparaison : le comment, le faisable... Aussi, je ne combats pas ceux qui posent la question et accrochent leur passion au niveau exaltant du projet historique. J'ai admis tout à l'heure que je pouvais me tromper, bien que je ne le croie pas. Il peut $\mathrm{y}$ avoir des forces en action qui agissent subrepticement et que je ne vois pas ou que, pour l'heure, je minimise. Vous avouerais-je que j'ai dans mes «petits cartons » les éléments de ce que j'appelle un « scénario de la dégringolade »... ? Ça s'est vu déjà dans l'histoire des situations où tout, presque, semblait contredire ou annuler une évolution soudaine qui s'est tout de même produite. Je me demande même si, depuis le printemps dernier, les risques fous qu'a pris le premier ministre du Canada dans la foulée du référendum pour consolider le régime fédéral ne sont pas une prémisse d'un tel scénario. Je réfère à mes textes récents sur cette initiative 2 .

\section{J. B. Ce serait intéressant de vous entendre sur ce « scénario de la dégrin- golade..." "}

G. B. Je vais vous en priver. D'abord ce serait trop long. L'identification exacte des acteurs et facteurs prendrait plusieurs pages. Et puis, l'enchaînement des propositions et situations hypothétiques ne pourrait s'établir en forme de ces propos. Retenons toutefois [27] que le départ de ce scénario est que l'indépendance n'est possible que par un enchaînement (aveugle) des carences de ceux qui pourraient l'empêcher et non par la poussée interne d'une dynamique centrifuge. C'est l'occasion de vous préciser que je ne suis pas en train de vous livrer tout ce que je pense de l'indépendance ou du destin collectif du Québec, mais de l'attitude géné-

2 Syndrome québécois et mal canadien, Québec, Les Presses de l'Université Laval, 1981, principalement les chapitres 30 à 39 et la Conclusion. 
rale que le citoyen Bergeron, que vous avez interpelé, a devant cette question toute fondamentale. De tout cela, nous pourrons en dire plus et plus explicitement sous les autres thèmes prévus pour ces entretiens.

\section{J. B. Est-ce que vous ne tenez pas un discours finalement démobilisateur pour la cause indépendantiste ?}

G. B. Ce n'est pas plus mon intention de démobiliser que de mobiliser les tenants de la cause contraire. À ces derniers, je tiens un langage encore plus dur selon les lignes suivantes : «Vous avez la soupe chaude depuis qu'un parti de l'indépendance a pris le pouvoir au Québec. Son contrat électoral de 1976 de ne pas faire la sécession à moins de mandat explicite est rassurant. Mais ce contrat, transitoire, il a pu le répéter quatre ans et demi plus tard. Attention ! Votre myopie et votre entêtement prolongé peuvent en arriver à créer à la longue cette dynamique politique du risque-tout. » Bien sûr, il y a l'économique et ses maux qui ont noms chômage, inflation, récession, faillites. L'indépendance n'est pas « tout », mais ce peut être aussi une façon de ré-envisager la totalité de ces choses.

\section{J. B. On sent votre sympathie d'instinct pour la cause de l'indépendance, mais votre insistance sur le comment...}

G. B. Aux Québécois gagnés ou sensibles à l'idée de l'indépendance, je leur dis d'abord qu'ils poursuivent le but le plus naturel qui soit, tellement naturel que ç'aurait dû être fait bien avant que nous ne devenions tous un peu psychosés à force d'en parler. Puis, je les amène [28] sans complaisance au plan d'une, ou de leur stratégie pour atteindre ce but. J'essaie de faire contrepoids à leur inclination psychique à atténuer les obstacles. Je ne gonfle pas ces difficultés. Je les préviens aussi que ces obstacles risquent de se durcir encore au fur et à mesure des progrès relatifs de leur cause. Pour cela, il faut se placer du point de vue de l'autre sans forcément épouser la contre-cause. 


\section{J. B. Et c'est là que vos analyses deviennent démobilisatrices.}

G. B. Pour ce qui est de la démobilisation, vous savez, il ne faudrait pas exagérer l'influence que j'ai... C'est quand même un service à rendre que de rappeler que, si l'indépendance doit avoir lieu, elle devra se faire en pleine connaissance de ces obstacles réels, sinon son projet s'essoufflera et finira par s'enrayer de luimême. Quels obstacles ? Même si nous devenions en situation de réclamer l'indépendance d'une seule voix collective, c'est-à-dire puissamment majoritaire, nous serons toujours la plus petite partie à la négociation « d'égal à égal ». Nous devrons forcer l'autre partie à y consentir. Une indépendance s'arrache ou s'octroie librement. Toutes ces conditions contredisent la seconde hypothèse. Il faudra donc arracher l'indépendance à la partie la plus forte. Tout pouvoir central, avec ou sans Trudeau, se reconnaît comme premier devoir le maintien de cette unité fédérale englobant le Québec.

Jouera aussi une solidarité très forte de toutes les régions du pays. C'est une erreur de voir le Canada comme une seule entité hors du Québec. Il y en a au moins quatre : le Canada des trois autres grandes régions puis celui qui fait l'unité d'ensemble dans la capitale fédérale. N'oublions pas la géographie : nous ne sommes pas à la périphérie qui est constituée par les maritimes, à l'aile la plus vulnérable du pays. Croyez-vous que le reste du Canada consente jamais à abandonner les provinces de l'Atlantique ? Et je ne parle pas de la force depuis toujours déterminante de l'Ontario, de celle de plus en plus grandissante de l'Ouest. Tout ce monde (sans compter une partie, qui [29] ne sera jamais négligeable, de Québécois) et ceux qui parlent en leur nom sont d'une totale unanimité sur le point que la séparation, le split, ne devra pas se faire. Comment allons-nous amener le plus gros, le plus fort en toutes hypothèses à composer?

\section{J. B. Mais des sondages répétés ont révélé qu'il y a une majorité de Cana- diens anglophones prêts à reconnaître l'autodétermination du Québec.}

G.B. Exact. Ils sont bien incapables de nier un droit aussi naturel. C'est là que commence l'ambiguïté fondamentale : générosité sur le principe de ce qui, selon 
eux, ne se produira pas ; mais, par ailleurs, refus de négocier si, contre toute attente, cela se produisait quand même ! Rappelez-vous leur réponse, lors du référendum, au projet d'association qui était quand même d'un bel esprit de conciliation. Cela tournait autour de ceci : "Vous nous en demandez trop. Nous ne pouvons pas vous empêcher de partir, mais assumez-en les risques. Nous ne sommes pas prêts à nous associer avec vous sur les ruines de ce que vous aurez brisé. Ne nous demandez surtout pas d'absoudre cet acte de sabotage du Canada ». Cela n'était pas que de la propagande référendaire. Bien, me direz-vous, si nous pouvions aller jusqu'au bout, les interlocuteurs des quatre ou des dix Canada anglophones seraient bien obligés de négocier un jour ou l'autre. À l'usure... Mais je vous laisse imaginer quelle serait la note collective à payer en attendant que ne se produise l'usure...

\section{J. B. Il s'agit tout de même d'un peuple civilisé, démocratique.}

G.B. Certes, presque autant que nous ! C'est dur à comprendre ce que nous voulons pour un cerveau en situation de pouvoir à Halifax, à Ottawa, à Toronto, à Calgary ou Vancouver. Je ne parle pas des esprits fanatiques ou intéressés par les espèces sonnantes. Je pense aux esprits les plus éclairés, aux intellectuels, [30] aux universitaires, aux artistes, des gens qui nous aiment pour notre spécificité même et qui admettent les injustices passées. Malgré leur mauvaise conscience, aussi relative que tardive, ils veulent nous garder avec eux parce que nous sommes l'élément culturel déterminant d'une différenciation d'avec les puissants voisins du Sud à la personnalité plus éclatante. Poussez-les au bout de leur sincérité, à l'aide d'un troisième ou quatrième scotch s'il le faut, ces gens dont certains se sont presque fait une carrière d'une connaissance empathique de notre groupe. Eh bien, leur réponse, qui finit par devenir ingénue avec la montée de l'indice d'alcool dans le sang, c'est qu'ils ne laisseront pas se réaliser cette charcuterie d'un pays qu'ils considèrent comme une belle construction historique. Pour notre plus grand bien, d'ailleurs, et pour leur fameuse identity, suffisamment difficile à établir pour qu'ils veuillent la maintenir à tout prix... Vous aurez noté que, jusqu'à maintenant, je n'ai pas parlé du recours aux armes économiques ni aux armes tout court. 
J.B. Il faudrait inverser l'adage : votre raison a ses raisons que le coeur ne connaît pas ! Car plusieurs de vos écrits témoignent qu'il y a du " nationaliste » en vous. Vous devez passer votre temps à le refroidir !

G.B. En tout cas, je le tiens en laisse. Il y a de la « déformation professionnelle » là-dedans, je suppose. Étudiant et enseignant l'histoire internationale des derniers siècles, j'ai vu comment naissent les nationalismes et comment se font les révolutions nationales. Il nous faut constater que si l'idée nationale a permis les plus nécessaires sursauts collectifs, elle a aussi rendu possibles les plus horribles folies de l'histoire, jusqu'aux racismes et fascismes contemporains. Le nationalisme peut devenir une idée dangereuse quand elle n'est pas suffisamment bridée. Dangereuse, sachons-le donc, mais aussi nécessaire dans des 1imites raisonnables pour des nations culturelles précaires en elles-mêmes, sinon menacées de l'extérieur. Pourquoi les grandes et vieilles nations, pour ne pas parler [31] des grandes puissances impériales, seraient-elles les seules légitimées à s'en réclamer ? Il y a une certaine dose de nationalisme combien nécessaire aux petites nations, surtout lorsqu'elles se retrouvent minoritaires dans des ensembles les intégrant.

Nous avons survécu par la force du nombre et la présence de l'Église qui ne nous a pas lâchés après la conquête. Aujourd'hui, la religion n'a plus cette valeur de sauvegarde. Et le nombre, nous le perdons petit à petit. Nous avons minorisé notre vainqueur pendant presque un siècle; puis, de la moitié de l'ensemble de la population, nous avons glissé au tiers, et maintenant à un quart fragile ; et les projections les moins pessimistes pour l'an 2000 ne sont guère rassurantes. On en arrive à se buter à un conséquent en forme de dilemme : ou faire des enfants pour garder le nombre, ou faire l'indépendance pendant que nous avons encore le nombre au Québec. Je m'étonne encore que la propagande indépendantiste ne frappe pas avec insistance sur ce clou de la conscience minoritaire : nous ne sommes pas une minorité à ce point malheureuse, mais nous ne voulons plus être une minorité. Point.

Mais alors, on retrouve les obstacles apparemment insurmontables à l'indépendance dont nous parlions. Et aussi les obstacles guère plus faciles à une reconquête du nombre. À l'époque de la pilule et de la faible immigration francophone, ce ne serait pas facile de s'en remettre à des politiques natalistes. Et je 
n'évoque pas les critiques génito-racistes que de telles politiques pourraient soulever. Au moins pour ce qui dépendait de nous, nous avons donné deux coups de barre avant qu'il ne soit trop tard. En nous donnant d'abord une politique d'immigration, avec quelque chance d'assimiler ceux qui choisissent de venir chez nous ; puis, avec une politique de la langue cohérente et courageuse (malgré quelques erreurs, comme la non-insertion de la « clause Canada »).

\section{J. B. On voit toujours que si l'analyste continue à penser à froid, le ci-} toyen éprouve à chaud les problèmes qui nous confrontent.

G. B. L'inconvénient avec le nationalisme, c'est qu'on ramène presque tout à la seule valeur nationale. À s'en crever les yeux. Tout n'est pas national si on agit dans un cadre national. Quand le nationalisme devient un problème, tous les autres problèmes s'y rattachent. C'est exagéré et déformant. Il y a aussi les valeurs de l'homme, les valeurs universelles de toute culture, les valeurs d'activité anationales comme celles de l'activité économique. De là, l'inclination naturelle à excuser trop facilement des déficiences, des carences et des échecs par l'idée nationale... insatisfaite. Depuis la Révolution tranquille, notre nationalisme n'est plus étroitement provincialiste, attardé. Il dégage une motricité collective. Il fait des choses, dont certaines étaient urgentes. Il faut continuer à tenir sous contrôle sa version péquiste. Les temps faibles et moroses de notre histoire coïncidaient avec un sentiment national plutôt faiblard ; les temps forts survenaient lorsque ce sentiment était vivace et « pratique » si j'ose dire. Vous voyez que le « nationaliste » que je suis, comme vous dites, ne fait pas que se méfier des pièges du nationalisme. Mais ce qu'il faut éviter par dessus tout, c'est de s'embarquer dans des embardées historiques.

C'est un des traits de notre nature humaine que d'excuser presque tout, y compris des échecs appréhendés, sous prétexte qu'on est passionnément sincère dans l'action qu'on mène, qu'on s'est engagé à fond de train pour le bon motif. Il faut plus. Il faut se mettre en position de pouvoir "gagner» de temps en temps ou petit à petit pour soutenir l'effort. Aussi pendant que j'insiste sur la relativité des causes - allant même jusqu'à soutenir que la façon de les servir vaut au moins autant que leur validité intrinsèque - je refuse de m'enfermer dans les cornes du di- 
lemme de la polarisation actuelle avec son double catastrophisme du fédéralisme qui étouffe et du séparatisme qui détruit. Je garde ma liberté d'apprécier, non seulement thèses et contre-thèses, mais de stimuler leurs tenants à mieux préciser leur option. À ceux qui posent en termes d'absolu ce préalable vital de l'indépendance du Québec, sans lequel tout est foutu pour le reste de l'histoire, il faut répondre qu'au contraire le pire est passé, que les réponses globales aux dangers non immédiatement politiques ne sont pas elles-mêmes [33] de nature politique, qu'il faut surtout être maître des opérations diverses à déclencher sur notre propre terrain.

\section{J. B. Autrement dit, c'est en intellectuel que vous nous mettez en garde contre une vue trop « intellectuelle " de notre condition présente et de l'his- toire à faire.}

G. B. Nous sommes tous d'incorrigibles bavards. Le fait de dire des choses, de les nommer, d'en verbaliser quelques-unes peut nous donner l'impression que nous les assumons, que nous les faisons avancer. Qui sommes-nous ? Un peu la mouche du coche... Elle est utile si elle sait bourdonner au bon moment et embêter l'oreille qu'il faut. Mais le chariot ou le coche, qui est-ce qui le traîne ? Beaucoup de gens, et pas des personnes comme nous, en première ligne.

J. B. L'autre jour, pendant un repas, alors que nous préparions ces entretiens, vous parliez avec abondance de l'importance que vous attachiez à l'émergence d'une nouvelle classe d'entrepreneurs industriels et de gestionnaires financiers et commerciaux.

G. B. Oui cela est aussi important que la refrancisation de Montréal, bien que peut-être moins spectaculaire. Ici, il faudrait rappeler l'effort des PME dans des régions types, la Beauce, le Lac Saint-Jean, l'Abitibi. Nous apprenons à faire des choses qu'on faisait peu ou qu'on faisait mal. Notre main-d'œuvre se spécialise, nos techniciens deviennent plus compétents, gestionnaires et entrepreneurs commencent à naviguer dans des eaux limitrophes. Et l'État a tout cela à l'œil, donne des coups de pouce utiles. Il y a là quelque chose. Surtout pour l'avenir. Pensez à 
l'extraordinaire retard que nous sommes en train de combler. Pour l'heure, cette vitalité nouvelle explique en grande partie que le Québec tienne assez bien son bout dans la conjoncture difficile des économies occidentales malgré l'instabilité constitutionnelle découlant de la prise du [34] pouvoir par un parti sécessionniste. Cette donnée toute nouvelle dans le paysage québécois, il faudrait pouvoir l'examiner de près non seulement en tant que phénomène économique mais pour ce qu'elle révèle en « substrat culturel » comme disent les sociologues.

\section{J. B. Ce nouveau leadership est-il susceptible de supplanter l'ancien ?}

G. B. Chose certaine, il y a une promesse dans ce sens-là. Nous avons été un peuple forcé de nous battre pour la langue et la culture. Il était inévitable que ceux qui pouvaient «parler »prennent le leadership. L'hégémonie des clercs a connu son apothéose dans la grande oeuvre de Lionel Groulx pour laquelle j'ai un respect profond et critique. La lettre des abbés Dion et O'Neil, en 1956, de même que les Insolences du Frère Untel, quatre ans plus tard, semblent avoir sonné le glas de cette hégémonie. Ils ont été remplacés par des espèces de clercs, dont nous sommes, des gens de parole et d'écriture qui discourent sur les pouvoirs mais qui ne sont d'aucun centre de décision. De grands absents des débats : les entrepreneurs industriels ou financiers. Est-ce qu'ils vont nous remplacer ? En partie. Mais ils vont surtout occuper un terrain complémentaire, leur terrain propre qui est de faire de la théorie économique appliquée, nous laissant celui de la théorie économique et de la théorie tout court.

\section{J. B. Cela reste un dialogue à deux interlocuteurs...}

G. B. Oh non! Tous les secteurs de la société devront y participer. Les mouvements de jeunes. Les mouvements de femmes, ce qui est beaucoup plus sérieux que le feu d'artifices des Yvettes, qui était ambigu par son affirmation de la négation. Les agriculteurs, par leur syndicalisme. Chez eux aussi perce l'idée d'entreprise. Ils parlent de plus en plus en termes d'organisation moderne, de croissance industrielle et non de [35] simple rentabilité. Et heureusement que nous avons 
sauvé les rares terres agricoles que nous possédions par la loi du zonage agricole ! J'aimerais entendre plus souvent toutes ces voix dans les média, sur la place publique. Ces gens écrivent peu, parlent peu, mais ils prennent des décisions qui comportent des conséquences. Ils pourraient contrebalancer la surpolitisation des vingt dernières années trop axée sur l'idée nationale et constitutionnelle. Cette surpolitisation a eu tendance à occuper l'ensemble de l'univers de notre mentalité collective, en quoi elle était nocive.

\section{J. B. N'êtes-vous pas en train de poser en quelque sorte la primauté de l'économique sur le culturel et le politique?}

G. B. Je prends acte de la réalité globale des vingt dernières années. Ce qui nous a dédouanés à l'extérieur, c'est bien autant la prise en main de nos richesses naturelles, en particulier hydro-électriques, que les objectifs culturels et éducatifs de la Révolution tranquille. L'Hydro-Québec n'est plus la chétive création de Duplessis mais un des plus gros entrepreneurs de l'Amérique du nord. Elle s'est illustrée mondialement par cette gigantesque réalisation de la Manicouagan et continue à le faire avec la Baie-James (à verser au crédit du régime Bourassa, à son sens du risque et de la prospective). Nos interlocuteurs ne sont pas seulement politiques. Bay Street compte bien autant que Queen's Park, Calgary, le quartiergénéral du pétrole, autant que la capitale Edmonton. Nos interlocuteurs sont aussi financiers (la rue St-Jacques, Wall Street, le marché des eurodollars). Ajouter les multinationales dont certaines ont leur siège social ici, et plus souvent une importante succursale./Bref, tout ce beau monde nous prend au sérieux par nos réalisations économiques. La force politique corrélative de la France, de l'Allemagne, du Japon réside dans leur capacité économique. Pas dans leur production culturelle ni dans le magnétisme de leurs tribuns politiques.

\section{J. B. Je ne m'attendais pas à cette insistance de votre part.}


G. B. Un jour, lors d'une conversation amicale sur une terrasse du boulevard St-Germain à Paris, un des grands noms de ma discipline, Bertrand de Jouvenel, un des fondateurs de l'art de la conjecture (ou prospective), se mit à dire en coq à l'âne : « Le plus tôt nous apprendrons à vivre à la japonaise, le mieux ce sera pour entrer dans le XXIe siècle. » Je me suis mis à penser à ce qu'est le Québec : six ou sept fois le Japon en surface, contrairement à lui doté de richesses naturelles, possédant une capacité d'accès aux technologies nouvelles au moins comparable à celle du Japon dévasté et vaincu de 1945. Je me suis mis à rêver à ce que ferait en peu de temps du Québec la classe dirigeante d'entrepreneurs japonais si elle s'en emparait. Imaginez! Ils ont battu les Américains et les Allemands sur leur propre terrain de la technologie de pointe. Je sais que c'est un rêve, presque une boutade. Bien aussi beau, cependant, que celui du fleur de lysé battant au vent parmi les 150 drapeaux des Nations Unies... Tout ça pour dire que nous ne sommes pas un peuple pauvre en potentiel. Nous avons un avenir.

\section{J. B. Qu'est-ce qui entraverait tant cet avenir si prometteur?}

G.B. Encore une fois ma réponse paraîtra un peu dénaturée pour un politologue. Je dis qu'avoir constamment le nez dans le dossier politique et constitutionnel peut devenir stérilisant à la longue. Si nous ne disposons pas des capitaux suffisants, nous jouissons d'un crédit important. Savons-nous nous en servir ? Ce slogan -« L'État, levier de notre économie » - dont il a été nécessaire de nous gargariser pendant une vingtaine d'années, doit maintenant être réévalué. L'État est un levier parmi d'autres ; mais c'est celui de la perspective du global permettant de voir la position relative des autres forces et d'agir éventuellement en politiques ponctuelles. Celui aussi qui profite de maux qui ne dépendent pas de lui, comme l'inflation. Il faut d'autres grands entrepreneurs. Déjà les HEC et les facultés universitaires d'administration forment un nouveau type de gestionnaires francophones, du type executive, ce qui veut aussi dire capables de passer à l'action. Il [37] ne s'agit plus que de former des cadres intermédiaires pour tenir la quote-part des francophones dans les entreprises étrangères.

\section{J. B. L'heure n'est pourtant pas à ces projections grandioses.}


G. B. Évidemment, je vais vite dans cette large parenthèse au moment où l'enregistrement sur cassette tire à sa fin. J'ai laissé tomber autant de choses que j'en ai soulevées. L'égalité des chances. La promotion des femmes. La structure de classes. Le syndicalisme. Le socialisme. Et la grande question : à qui tout cela profite ? Qui doit profiter ? (Gardons-en pour une prochaine fois).

\section{J. B. Revenant au propos principal, de tout cela, ne se dégage-t-il pas aus- si un rôle social à revoir pour la catégorie des intellectuels dont vous êtes?}

G. B. Certainement. Et c'est très heureux. Je vis à l'intérieur de l'université, de « ses pompes et de ses oeuvres » depuis 1944. J'ai besoin de voir et d'entendre des gens d'autres milieux. Même cela constitue pour moi un petit retour aux sources. Je ne veux pas profiter de ces entretiens pour raconter ma vie personnelle, anecdotique, qui n'a pas de relief particulier. Je signale seulement que je suis d'une famille d'hommes d'affaires. Mon père et ses deux frères ont passé leur vie dans les affaires. Je sais la qualité de cette activité si différente de la nôtre. Mais je ne veux pas jouer à l'intellectuel qui, paradoxalement, se magnifie en s'abaissant ou en se méprisant officiellement... Je trouve même indécent de se donner des airs de fausse démagogie sur la place publique et de vouloir passer pour du « monde ordinaire ». Nous ne sommes pas du monde ordinaire pour la simple raison que nous manipulons des idées. Dès lors, nous vivons un peu en retrait de la société. Nous entretenons entre nous une image déformante de la réalité. Par ailleurs, l'intellectuel est un être d'inquiétude, de changement, [38] de progrès. Nous sommes de gauche, plus à gauche, en tout cas, que la société dont nous sortons et que nous représentons plus ou moins bien à cause de cela même. Surtout que la classe des intellectuels est un peu trop gâtée dans ce pays, en particulier les universitaires. Nous menons une vie presque trop facile, sans sérieuses limitations financières, un peu en dehors du struggle for life du citoyen de plus moyenne condition. Fautil craindre que d'autres catégories sociales, la jeunesse principalement, portent un jour un jugement peut-être sévère sur ce que nous aurions dû faire, ou dû faire autrement? 


\section{J. B. Que reste-t-il finalement comme fonction propre des intellectuels ?}

G. B. De faire accélérer les choses, de vouloir aller plus vite que les autres, et d'en expliquer le pourquoi et le comment et au moins autant celui-ci que celui-là ! N'avons-nous pas été les premiers à sonner le réveil sous le dernier mandat Duplessis ? Et il faudra toujours des artistes pour dire les beautés de Terre-Québec. Déjà, à leur façon, commerçants et industriels la célèbrent par la profusion des raisons commerciales avec le suffixe en «... bec ». L'avez-vous remarqué ? Mais à Sidbec, lancé par l'État et qui est presque en faillite, on peut préférer Provibec, qui est en pleine expansion. 
[39]

GÉRARD BERGERON.

de l'autre côté de l'action.

$2^{\mathrm{e}}$ entretien

L'UNIVERSITAIRE

\author{
«... naturellement, \\ en toute nécessité »
}

$\underline{\text { Retour à la table des matières }}$ 


\begin{abstract}
J. B. Comment et pourquoi choisit-on d'étudier et plus tard d'enseigner les sciences sociales dans le Québec d'après-guerre ? C'est un champ universitaire nouveau (la Faculté de Laval a officiellement été fondée en 1943, soit un an avant votre arrivée), assez peu connu encore par la jeunesse de l'époque.
\end{abstract}

G. B. Cela s'est fait naturellement, en toute nécessité, oserais-je dire. Si la Faculté n'a qu'un an, en septembre 1944, elle a quand même été précédée par l'École des sciences sociales à partir de 1938. À cette époque du milieu des années trente, je suis au début de mes études classiques. Je manifeste déjà un intérêt très vif pour l'actualité politique internationale. N'oublions pas que cette époque marque les guerres d'Espagne et d'Éthiopie, les coups de force de Hitler, ses derniers préparatifs avant Munich en 1938 et l'invasion de la Pologne en 1939. On n'échappe pas à cette atmosphère tendue, angoissante dont nous parlent les journaux. J'écoute la radio, regarde les actualités cinématographiques. J'avais aussi développé au collège un grand intérêt pour l'histoire générale et universelle : les grandes étapes de l'humanité, les révolutions, les idéologies, les systèmes économiques. Par-dessus tout, j'aime écrire. Je rêve même à une vie d'écriture. Dès les premières années du cours classique, les rédactions ou narrations sont des fêtes. Elles ont la longueur de nouvelles ou de petits romans. Je transporte cette démangeaison d'écrire, durant les années [42] terminales, dans les dissertations littéraires, historiques ou philosophiques d'une taille encore inédite. C'est resté légendaire.

La bibliothèque du Collège de Lévis ne suffit pas, je me documente à celle du Parlement à Québec. Comme ma calligraphie est mauvaise, je dois dactylographier mes textes. Au réfectoire du collège, un soir de 1938, je lis dans L'Action catholique (le seul journal permis, Le Devoir était simplement « toléré »; certains étudiants pouvaient le lire grâce à des amis prêtres qui, eux, y étaient abonnés) l'annonce de la fondation de l'École des sciences sociales sous la direction du Père Georges-Henri Lévesque. J'ai aussitôt dit à mes voisins de table : « Ça m'étonnerait beaucoup si, a l'université, j'allais ailleurs que dans cette école ». 


\section{J. B. Pour y faire quoi ? En aviez-vous déjà une idée précise ?}

G. B. Oui et non. La guerre se déroule, et mon intérêt, déjà plus grand que celui de la moyenne des gens d'ici, augmente pour cet extraordinaire événement qui remplit toute l'époque. Je m'y intéresse non pas à cause de la prospérité économique qu'elle nous a value du jour au lendemain, ni même à cause de la crise de conscription plus tard, mais bien comme phénomène universel qui allait conditionner le reste de nos existences. Voyez l'extraordinaire coïncidence d'un adolescent s'éveillant à la vie de l'esprit tout en ayant une conscience de plus en plus vive d'un événement d'une si grande et tragique ampleur : c'est de cela, et avec toutes les conséquences qui pointaient déjà, qu'il faudra être témoin le reste de sa vie comme programme intellectuel.

J'ai parfois regretté de ne pas avoir mis par écrit cet état d'esprit. L'expression en aurait été probablement plus maladroite que la manière avec laquelle je vous en parle après toutes ces années ; mais elle aurait une fraîcheur, une authenticité dans l'expression que je ne retrouve pas. Je suppose que d'autres jeunes gens de l'époque ont vécu le même intérêt. Si j'en parle, ce n'est pas seulement que cela a été à l'origine de tout, mais bien parce que j'ai continué.

[43] Inutile de vous dire avec quelle intensité j'ai vécu les faits principaux de cette guerre, ses grands tournants. En rétrospective, deux souvenirs s'imposent avec précision : la chute de Paris en juin 1940, sa libération quatre ans plus tard en août 1944. On ne comprenait pas dans mon entourage que ça me fasse pleurer ! Je me souviens de m'être passionné pour de grands thèmes : Cette guerre continue-t-elle la précédente ? L'intervalle des vingt ans (1919-1939) n'avait-il pas été autre chose qu'un armistice prolongé ? Et avec la découverte et l'application de l'énergie atomique, ça devenait une question d'actualité que d'avoir à s'interroger sur rien moins que le sort de l'espèce humaine.

\section{J. B. J'imagine que vous étiez un grand consommateur de journaux ?}


G. B. Dans mon désir et mon besoin d'écrire pointe déjà une formule, un métier, un style de vie : le journalisme. Il n'y avait guère que les auteurs de radioromans et les journalistes pour vivre de leur plume en ce pays et, en ce dernier cas, plutôt mal. Ce journaliste, je le vois alors comme commentateur-éditorialiste de l'actualité. C'est donc moins le journaliste véritable qui va chercher la nouvelle et qui la fait, que celui qui jette un regard contemporain d'analyse sur l'évolution de la société.

J. B. Mais on n'enseigne ni le journalisme ni les sciences politiques à la jeune Faculté des sciences sociales de l'Université Laval, en septembre 1944.

G. B. C'est vrai. Mais la sociologie était ce qui se rapprochait le plus de mes objectifs de carrière. Et je me disais qu'à l'occasion je prendrais quelques cours de lettres. Il faut savoir que la Faculté des lettres était alors plutôt stagnante, sans débouchés pour ses étudiants qui appartenaient au clergé ou à des communautés religieuses pour la plupart, quand ils ne venaient pas [44] de l'extérieur. Ce n'était pas très attirant pour un jeune.

\section{J. B. Parlez-nous de la Faculté des sciences sociales de l'époque.}

G. B. L'image qui m'en reste est essentiellement celle d'une équipe. Au centre, son animateur, dès ce moment un homme de prestige, le Père Georges-Henri Lévesque, dominicain. Il était allé étudier les questions sociales en Europe, à Lille spécialement. L'histoire retiendra que son oeuvre principale aura été d'avoir acclimaté au niveau universitaire les sciences sociales, essentiellement critiques et novatrices, dans un milieu qui était encore prédisposé à se satisfaire d'une philosophie dogmatiste et d'une morale passéiste. Je rappelle que déjà il avait lancé ce type d'études à l'intérieur de son ordre, plus précisément à l'Institut dominicain de philosophie d'Ottawa. Il avait aussi donné quelques cours du soir à l'École des sciences sociales de l'Université de Montréal, fondée et dirigée par Édouard Montpetit. L'Université Laval lui avait aussi demandé de venir enseigner à son « école du soir » dans les années trente. À la fin de mes études classiques, j'étais 
venu assister à quelques-uns de ses cours pour m'initier à l'étude des sciences sociales.

Ce dominicain était destiné à devenir l'agent principal de la reconnaissance de plein droit d'une faculté universitaire des sciences sociales. Il a déjà confié qu'il voyait d'abord cette Faculté à Montréal, métropole et capitale industrielle, plutôt qu'à Québec, capitale administrative. Mais c'est à Laval que le terrain mûrit d'abord et, en grande partie, sous le patronage prestigieux du Cardinal Villeneuve.

\section{J. B. Mais le Père Lévesque n'était pas seul ?}

G. B. Certes non. Aux côtés du Père Lévesque, il y avait un homme remarquable par son effacement, sa sagesse, son sens de la culture universitaire et de la recherche, [45] le Père Gonzalve Poulin, franciscain. Il a été le premier directeur des études de cette faculté, en 1943, et le directeur de la première école rattachée à la Faculté, l'École de service social. Les premiers cours d'économique furent donnés par Paul-Henri Guimond, d'autres par Jean-Marie Martin qui allait plus tard succéder au Père Lévesque comme doyen. Mais surtout, à mon arrivée, une brochette de jeunes professeurs de la première fournée de diplômés de 1941, qui revenaient d'un séjour d'études aux États-Unis, l'Europe étant inaccessible à cause de la guerre : Maurice Lamontagne, formé en économique à Harvard ; Maurice Tremblay, professeur de philosophie sociale et politique, et qui rentrait de Harvard aussi ; Jean-Charles Falardeau qui avait poursuivi sa formation à Chicago et qui sera l'instigateur de l'étude de la sociologie au Québec ; Roger Marier avait, pour sa part, étudié le service social à Washington. D'autres étaient allés en Ontario : Albert Faucher rentrait de Toronto où il s'était formé en histoire économique ; l'abbé Gérard Dion reviendra au bercail l'année suivante, après un stage d'études en relations industrielles à Queen's. Deux n'étaient pas allés étudier à l'étranger: Eugène Bussières, premier responsable du Conseil supérieur de la Coopération, et Jean-Pierre Després, un de nos spécialistes en relations industrielles, qui aura, par la suite, une longue carrière internationale à Genève, au Bureau international du travail, tandis que Bussières en fera autant au siège de l'Unesco à Paris. Bref, cela constituait une jeune équipe animée par la conviction de fonder véritablement les sciences sociales dans notre milieu. 


\section{J. B. Vous avez vécu cet élan des sciences sociales dans le Québec d'après- guerre. Comment expliquer que la pénétration des sciences de la société ne se soit pas produite plus tôt ?}

G. B. Il a fallu la crise économique pour les justifier. La conscience des problèmes sociaux causés par la crise a donné naissance tout naturellement à l'École de service social. Le fondement de la crise de même [46] que la solution du Welfare State étaient d'ordre économique. La question ouvrière se révélant alors dans toute son ampleur et sa complexité, les départements de relations industrielles - un calque de l'anglais : industrial relations - sont apparus. La sociologie répondait à un besoin pressant de connaître le fonctionnement des sociétés modernes. Avec Toronto, Queen's et McGill, Laval a été l'une des premières universités à enseigner officiellement les sciences sociales pendant et au sortir de la guerre.

\section{J. B. Pourquoi la science politique ne se trouve-t-elle pas parmi ces dépar- tements originels?}

G. B. Pour un ensemble de facteurs. D'abord, un facteur global qui renvoie à ce que je viens de dire : la génération du Père Lévesque s'éveille à la « chose sociale » dans le sillage de la crise économique ; elle est attirée par les vocations sociales principalement : syndicalisme, coopération, éducation populaire, service social. Ce caractère prédominant du social intégrait l'économique et était à toutes les faces : patronale, syndicale, industrielle, urbaine, familiale, d'assistance sociale, d'organisation coopérative. La société devenait quelque chose sur lequel on peut agir. D'autre part, nous sommes en plein duplessisme. Le mot même de « politique » connotait quelques chose de suspect, voire de dépravé. On n'en parlait jamais que de façon négative, "La politique » disait-on du bout des lèvres... Il aurait été même suspect de songer publiquement à fonder un département consacré à cette « chose ». Quand on fondera cet enseignement en 1954, il faudra employer le pluriel - le « département des sciences politiques.. » - pour désamorcer la dynamite du mot, selon le modèle de la traditionnelle École des Sciences politi- 
ques de Paris, et ajouter - « ... et administratives » - comme précaution supplémentaire ! Le Département des sciences politiques et administratives naîtra donc onze ans après les départements d'origine.

Nous aurions quand même pu naître plus tôt. Le Père Lévesque y portait un intérêt particulier, avec [47] son sens politique très aiguisé. Le manque de ressources internes autant que les rapports avec le pouvoir politique expliquent ce retard. Le Père Lévesque m'avait invité comme observateur - une marque du sérieux de son pari sur un jeune - à une réunion préliminaire, tenue en 1946 ou 1947, groupant ceux qui seraient éventuellement intéressés à la mise sur pied d'un tel département. J'étais alors étudiant en troisième année.

\section{J. B. Cette dévalorisation de la politique s'exprimant par une maximisa- tion du social est particulière au Québec d'alors. C'est donc dire qu'au Ca- nada anglais, la science politique est davantage contemporaine des autres sciences sociales.}

G. B. C'est vrai pour Queen's, Toronto, McGill et Vancouver, où la science politique était assez reliée à l'enseignement de l'économique, et aussi à l'histoire. Ces universités produiront ainsi les premiers grands administrateurs anglophones de la Fonction publique fédérale. Nous n'en fournissions pas puisque nous n'en formions pas. Et la Fonction publique fédérale allait devenir plus tard le principal débouché pour les premiers diplômés que nous formerons, celle du Québec sous Duplessis étant assez peu accueillante, pour employer un euphémisme. Après une année d'introduction, je m'inscris donc en sociologie un peu par défaut.

C'était le département qui m'apparaissait le moins éloigné de l'étude des relations internationales. Mais aussi avec une conviction progressive que c'était une bonne décision que j'aurais pu prendre par choix. Nous étions en contact avec les derniers développements de la sociologie américaine, par de jeunes professeurs qui venaient de se former aux Etats-Unis, et d'autre part, notre culture française nous donnait un accès direct aux grandes oeuvres de la sociologie française et à des ouvrages traduits des sociologies allemande et italienne. Malgré de faibles effectifs professoraux (c'était le cas dans tous les autres départements), nous jouissions d'un fort degré [48] d'autonomination du programme de la discipline, 
contrairement à l'enseignement de la sociologie en Europe et singulièrement en France, où elle était encore tenue pour une des spécialités de la philosophie. À cette époque, la sociologie ne jouissait d'aucune autonomie universitaire, coincée qu'elle était entre la philosophie et l'ethnologie. Il n'existait pas encore cette réalité, à l'américaine, d'un département de sociologie. Nous étions, en 1944, un département qui parlait français dont ses jeunes professeurs pouvaient se réclamer aussi bien des maîtres européens de la « science sociale » que des grands courants plus récents de la sociologie américaine. Ce mixage était inédit. La sociologie américaine avait franchi des pas prodigieux depuis le début du siècle en raison de problèmes de société que posait l'intégration culturelle américaine.

Je piocherai le droit public et tâterai de l'histoire internationale presque en même temps. Je me collète à cette jeune et vigoureuse sociologie américaine, ainsi qu'à une tradition européenne dont je me réclame toujours : Montesquieu, Comte, Tocqueville, Marx, et ces deux autres colosses que sont Émile Durkheim et Max Weber. Je m'ouvre ainsi à l'histoire par la perspective, très large, de la sociologie historique et de la philosophie sociale. C'était emballant.

Mes premiers travaux sérieux d'étudiants - mémoire de deuxième année et thèse de troisième - porteront sur de grands sujets contemporains de relations internationales. Mon mémoire consistera en une étude en parallèle des deux guerres mondiales s'efforçant de faire la démonstration que la deuxième fut plus « mondiale » que la première quant à l'origine, la stratégie et les théâtres d'opérations, les enjeux idéologiques et impériaux. C'était naïf de s'attaquer à pareil sujet dès 1946 ! Disons que j'éprouvais le besoin de situer l'époque où nous entrions. Le sujet de la thèse était à peine plus restreint. Il portait sur une analyse en parallèle encore ! - de l'oeuvre de la Société des Nations en matière de « sécurité collective » en comparaison des mécanismes de « maintien de la paix » que venait d'établir l'Organisation des Nations Unies en 1945. C’était encore une tentative de se situer, mais cette fois-ci à l'horizontale, dans son époque !

J. B. Mais vous êtes étudiant en sociologie. Ces matières relèvent de l'histoire internationale et du droit international. Qui vous les enseigne ? 
G. B. Il n'y a aucun enseignement en ces matières. Je me débrouille tout seul avec les ressources de la bibliothèque de Laval que complètent celles de la bibliothèque du Parlement. Mais je dois beaucoup à mes professeurs qui ont toléré que je m'embarque, seul et avec les moyens du bord, dans de pareils sujets : à Maurice Tremblay et au regretté Jean-Charles Bonenfant, qui ont été mes premiers lecteurs attentifs ; à Jean-Charles Falardeau qui, voyant en moi un futur sociologue patenté, a eu le libéralisme de me laisser choisir les relations internationales. Je dois beaucoup aussi au Père Lévesque. Il m'a procuré cette chose inestimable pour un jeune homme : une certaine dose de confiance en soi. C'est ce qu'il m'a fait sentir aux bons moments.

Un autre homme dont je veux évoquer la grande figure est celle du Père JeanThomas Delos, dominicain, un homme de réputation internationale en sociologie et en droit public, qui avait été un des professeurs du Père Lévesque à Lille. Il avait passé la plus grande partie de la guerre à enseigner à la Faculté des sciences sociales, coupé de la France par l'occupation nazie. Le collégien de Lévis avait assisté, de façon très anonyme, à ses conférences publiques. C'était mon premier contact avec un maître authentique, expérience qui deviendrait rarissime par la suite. Hélas ! au moment de m'inscrire à la Faculté des sciences sociales en septembre 1944, il venait d'être appelé pour agir comme consultant spécial auprès de Jacques Maritain, nommé ambassadeur spécial auprès du Saint-Siège. Mais cet énorme désappointement allait être compensé par sa venue à Québec pour trois ou quatre semaines au dernier mois de ma dernière année d'études, au printemps 1947. Vous dire avec quelle intensité j'allais recevoir cet enseignement! Ce contact tardif avec ce maître (que je poursuivrai par la lecture attentive de ses ouvrages) allait confirmer ma propre vocation de chercheur dont je voyais mieux maintenant la double assise, sociologique [50] et juridique. Comme étudiant, je me considérais fort privilégié de bénéficier de l'estime intellectuelle de cet homme qui s'ajoutait à celle déjà presque « professionnelle » de mon doyen.

\section{J. B. Dans votre esprit, la carrière universitaire est-elle en train de se substituer à celle du journaliste ?}


G. B. J'avais fait part à mon doyen de mon intérêt très vif pour le journalisme, dès le début. Il m'avait encourage. Comment concilier cet encouragement avec le fait qu'il comptait sur moi comme professeur et qu'il voulait m'aider à aller faire des études spécialisées en Europe ? Son mode d'argumentation était simple : la carrière universitaire n'exclut pas celle du journaliste, me disait-il ; à point nommé, elle me permettrait un saut, relatif ou partiel, dans le journalisme. C'était déjà clair à l'époque et ça allait se produire ainsi une dizaine d'années plus tard, en 1956, dans des circonstances que nous dirons plus loin.

Dès mes études, je me suis fait la main par des articles de revues et de journaux avec la bénédiction du Père Lévesque, si vous me permettez l'expression, qui avait établi des contacts pour moi. D'abord, il m'avait invité à collaborer à la Revue dominicaine, l'ancêtre de la revue Maintenant également disparue : j'ai écrit cinq ou six textes de politique internationale dans cette revue. Je me rappelle aussi d'un autre sur Montesquieu dans la Revue de l'Université Laval. Les articles de journaux étaient bien autrement nombreux.

En 1945, Léopold Richer fondait un hebdomadaire culturel, social et politique appelé Notre Temps. Richer était correspondant parlementaire du Devoir à Ottawa, un rôle clé pendant la guerre. Son nationalisme n'était pas très accordé à celui qui prévalait à la Faculté des sciences sociales, mais il entretenait de bonnes relations personnelles avec le Père Lévesque. Celui-ci lui parla de moi. Richer accepta et publia volontiers mes premiers papiers. J'en ai écrit une trentaine en tout pour son hebdomadaire. Mes tout premiers soulevaient la question de la liberté d'information dans l'Europe d'après-guerre, menacée par l'hégémonisme des [51] grandes agences de presse américaines. Voyez, c'était déjà un sujet-carrefour de tous mes intérêts de l'époque jusqu'à aujourd'hui. J'ai fait cette recherche à l'été 1946, à l'Université Columbia où je suivais des cours de summer session en vue de me préparer à mes études européennes. J'ai beaucoup apprécié le cours sur les relations internationales de Frederick L. Schuman, une sommité universitaire en la matière.

J. B. Ainsi donc, vous avez un premier contact avec une grande université américaine avant l'Europe. 
G. B. Ce stage d'été m'a permis de découvrir New-York. Je voulais connaître cette synthèse de l'Amérique avant mon départ pour l'autre continent. Ce fut l'objet d'un article dans Notre Temps dont mon professeur Falardeau se servit dans un cours de sociologie urbaine * . Je ferai d'autres articles sur et à partir de Newyork : sur le théâtre, sur le cinéma en particulier. Le premier grand film shakespearien de Laurence Olivier, Henry the Fifth, avait été présenté en grande première sur Broadway, ce qui m'avait permis d'en parler à mes lecteurs longtemps avant qu'il ne soit présenté au Canada. L'autre hit cinématographique était le célèbre Rome ville ouverte d'un cinéaste italien, jusque-là inconnu, Roberto Rossellini.

\section{J. B. Le Carabin, qui était l'équivalent du Quartier latin à l'Université de Montréal, vous y avez aussi correspondu, l'avez dirigé même ?}

G. B. Oui. Et par le fait même, j'ai un peu connu Jacques Hébert et Jean-Louis Roux, respectivement directeur et rédacteur-en-chef du Quartier Latin. L'année où j'ai dirigé Le Carabin, j'ai mis la main à la pâte à toutes les phases de la production, y compris des incitations à la collaboration. J'apprendrai donc très tôt qu'il y a des tâches plus pénibles que celle d'écrire soi-même, soit [52] particulièrement celle de faire écrire les autres ! Et je dois à la vérité de vous dire que l'année où j'ai dirigé ce journal, mon rendement académique s'est trouvé quelque peu diminué par ce travail journalistique intra et extra muros au Carabin et à Notre Temps.

\section{J. B. Le journal étudiant était contestataire à l'époque ?}

G. B. Oui, et avec de petites pointes de radicalisme ici et là, mais toutefois moins qu'au Quartier latin, la quiétude de la «vieille capitale » et de sa population de fonctionnaires s'y prêtant moins. Si le journal fut suspendu avant la fin de l'année, je m'empresse d'ajouter que ç'avait été le cas de toutes les activités étudiantes. Lors de la fête universitaire annuelle, des étudiants, dans l'esprit « cara-

* Voir Petite géographie personnelle, document no. 1 
bin », avaient vidé un bordel de luxe du Vieux-Québec ; mais ce n'avait pas été à l'incitation du journal. Il serait plus juste de dire qu'ils avaient sauvagement fait le sac de la « maison » en question. Il y eut une réaction violente de la part des intérêts financiers derrière ce commerce. Chauffeurs de taxi en tête, ils ont « cassé de l'étudiant » des jours durant dans la Haute-Ville, dans les restaurants et tavernes, à l'aide de chaînes et de bâtons de baseball. Ça n'a pas été une petite affaire ! L'Université avait alors suspendu toutes les activités étudiantes en signe d'apaisement, y compris le journal. Il y avait un substrat « lutte de classes » dans cette affaire.

J. B. En septembre 1947, vous traversez en Europe pour un long stage d'étude : un an à l'Institut des Hautes Études Internationales de Genève et deux ans à la Faculté de droit de Paris. Étiez-vous des premiers Québécois d'après-guerre à aller parfaire leurs études en Europe ?

G. B. Presque. Quelques étudiants avaient fait le voyage l'année précédente par cargo, avec des moyens de fortune. J'étais des premiers voyages réguliers en partance de Montréal-Québec pour Liverpool.

[53]

\section{J. B. Pourquoi avoir choisi Genève?}

G. B. D'abord parce que c'était la ville internationale par excellence. S'y trouvaient en particulier, à quelques pas de l'Institut, l'édifice du Bureau international du Travail et, un peu plus loin, le grand Palais de la Société des Nations qui se ranimait en devenant siège européen de l'O.N.U. Imaginez combien ce contexte pouvait paraître merveilleusement irréel et historiquement flagrant au petit Canadien français que j'étais ! Genève aussi pour des raisons plus pratiques ; en cette Europe dévastée, la Suisse permettait des facilités de ravitaillement et de logement bien supérieures à celles de la France. Cette première année en fut une de recyclage intensif. J'entre dans des disciplines bien tranchées ; droit international, histoire diplomatique, économie internationale, etc. Je n'étais pas habitué à ce 
découpage traditionnel. Ce n'était qu'aux États-Unis que les relations internationales étaient enseignées comme telles, comme une matière spécifique.

\section{J. B. Et votre journalisme devient lettre morte ?}

G. B. Pas du tout. Pendant cette première année de séjour en Europe, nous collaborons sur une base hebdomadaire, mon épouse, Suzanne Patry, et moi, à $L a$ Patrie du dimanche : récits de voyage, observations de la vie quotidienne s'entremêlaient à des articles d'analyse internationale. Il est bon de rappeler que cette année 1947-48 est celle du déclenchement de la guerre froide : doctrine Truman du containment, Plan Marshall, le Kominform, puis le coup d'État de Prague, le Blocus de Berlin, les prémisses de l'OTAN, etc.

\section{J. B. Puis, vous vous installez à Paris.}

G. B. Après un an de ce régime universitaire à Genève, je décide de m'inscrire aux études de doctorat en droit [54] international à Paris *. Remarquez que je n'avais pas fait des études régulières dans une Faculté de droit. J'abordais donc ces études par le secteur du droit peut-être le plus difficile, le plus primitif, le plus incohérent ; je l'abordais par le biais analytique de la sociologie du droit, à l'envers donc du mode de raisonnement déductif de cette science juridique qui procède à partir de définitions et de propositions. Inutile d'ajouter que ça n'a pas été facile d'absorber toute cette matière et de préparer des examens de doctorat.

\section{J. B. Des examens, mais pas de thèse ?}

G. B. J'en mets une en marche. Et voici que se manifeste encore ma manie des grands sujets ! La grande question de l'heure, au moment du lancement de la guerre froide, c'est le contrôle international de l'énergie atomique à des fins pacifiques.

* Voir Petite géographie personnelle document n $\mathrm{Il}$. 
Deux plans s'imposent qui s'excluent : l'américain et le soviétique. Ça m'apparaît le sujet de l'heure. Je l'inscris. Il m'oblige à faire, d'abord l'historique du désarmement à travers les siècles, depuis l'arbalète jusqu'à la bombe atomique, et à passer au crible tous les traités de paix pour voir quels organismes de contrôle étaient prévus.

Peu à peu, je découvre qu'il manque un cadre analytique pour étudier ce phénomène. Comment peut-on instituer par hypothèse un contrôle politique sur une question aussi vitale, délicate et planétaire en même temps ? Je me dis : il faut penser à un ordre politique moins anarchique, mieux organisé. Cet ordre politique ne pouvait être que l'État moderne, « rodé »depuis quelques siècles. Ma naïveté et mon ignorance étaient incommensurables, mais belles ! Me voilà donc en train d'écrire une introduction théorique pour laquelle j'essaie de démonter les grands mécanismes, du fonctionnement interne des États afin de discerner comment ils pourraient se comporter entre eux en déléguant de leurs pouvoirs à un organisme international, éventuel détenteur d'une souveraineté mondiale. [55] Ce travail m'a complètement noyé dans mon sujet ! Cette introduction est passée de vingt à quatre-vingt-dix pages puis à deux cent cinquante pages ! J'étais en train de faire a contrario la preuve de la nécessité dune théorie de l'État à élaborer..

Quand je rentre en 1950, je traîne par devers moi un gros manuscrit inachevé et qui pousse dans toutes les directions. Il va dormir dans un fond de tiroir pendant quatre ans alors que j'en reprendrai des éléments dans un nouvel enseignement sur une présentation générale à la science politique. Voilà l'origine du premier de mes livres qui sera l'objet de ma thèse de doctorat en 1964 et qui sera publié, l'année suivante, à Paris, sous le titre de Fonctionnement de l'État.

J. B. Et dont Raymond Aron, le préfacier, en le présentant comme opus maximum, dira qu'il « illustre avec éclat la vocation culturelle du Québec ». En septembre 1950, vous commencez donc votre carrière d'enseignement à l'Université Laval, alors situé dans le Vieux-Québec. Quel souvenir gardezvous de ces débuts?

G. B. Je le dis tout net : le plus beau des souvenirs, le souvenir impérissable d'une liberté intellectuelle extraordinaire et d'une amitié chaleureuse entre collè- 
gues qui sont aussi des amis. Sous l'égide du doyen, qui se trouve être aussi « le Père », nous formions une espèce de famille. Il s'y produisait certains grincements, mais pas plus que dans « les bonnes familles »! La Faculté comptait seulement une quinzaine de professeurs ; j'ai été le onzième ou le douzième à être nommé. Dans ma première classe, des étudiants s'appelaient, entre autres, Claude Morin, Gérald Fortin, Yves Martin, Fernand Dumont, qui se sont fait un nom comme vous voyez.

Très nettement, dès le départ, nous avions conscience d'être des initiateurs d'un segment important de la vie intellectuelle d'ici. Moi, j'étais l'homme de la politique internationale et, de façon générale, de toutes les relations internationales, y compris l'histoire. C'était démentiel pour un jeune professeur d'avoir à [56] couvrir pareil terrain! Les effectifs professoraux réduits obligeaient à ces voltiges intellectuelles. Moi, j'étais ce petit Canadien français - nous ne disions pas encore Québécois - produit d'une culture à maints égards provincialiste, qui venait dire à des cadets de quelques années : «Voilà le monde dans lequel vous habitez; nous ne sommes pas seuls sur cette terre ; il faut enlever nos œillères, prendre conscience que le Canada, la première des » moyennes puissances «, se trouve juste situé entre les deux Supergrands ». Voilà ce que je disais à ces jeunes gens que rien dans leur sensibilité ou dans leur formation antérieure ne prédisposait à une vue planétaire ! Et encore : «Si jamais il y a une explication, atomique ou pas, entre les deux Grands, ça risque de se passer sur le sol canadien ; une ligne droite Moscou-Washington passe sur le Québec ; nous pourrions être la Belgique de cette guerre », qui, fort heureusement, n'a pas encore éclaté.

\section{J. B. Cet effort pour impliquer vos étudiants dans la politique internatio- nale produisait-il des résultats?}

G.B. Oui, je pense. Il ne s'agissait pas de produire des internationalistes à la chaîne, mais d'ouvrir des horizons à un moment où l'humanité prenait conscience de son unité : One World, selon le titre du livre célèbre de Wendell Wilkie, le candidat malheureux devant Roosevelt aux élections présidentielles de 1940. J'enseigne à partir de septembre 1950. En juin avait éclaté la guerre de Corée, qui sera marquée par des épisodes d'une excessive gravité : l'intervention massive des 
Chinois qui refoulent les Américains à la mer, la demande à ce point insistante de MacArthur de recourir à l'arme atomique contre la Chine que Truman le limoge. À la fin de mes cours d'Histoire des relations internationales et de Problèmes contemporains, je consacrais une vingtaine de minutes à commenter l'actualité à partir des manchettes des journaux. Dans le cadre de ce dernier cours, je pense bien avoir été, dès 1952, le premier professeur à traiter du phénomène du sousdéveloppement à l'échelle mondiale.

\section{J. B. De tels cours devaient être tout un « choc culturel " pour ces jeunes gens, frais émoulus de nos collèges?}

G. B. Et dont certains se souviennent encore. Parfois nous formions un petit groupe et, l'ambiance chaleureuse du Vieux-Québec y invitant, nous allions continuer ces conversations après le cours, dans quelque taverne des environs. On pouvait se sentir en une espèce de symbiose intellectuelle, car les étudiants nous apprenaient aussi des choses! Ç'a été une période d'or de ma vie. Dans une émission-fleuve de cinq heures à Radio-Canada, il y a quelques années, Fernand Dumont et moi l'avons évoquée avec ferveur et bonne humeur. Des auditeurs nous le rappellent encore à l'occasion, ainsi qu'à Roger Nadeau qui avait pris cette initiative.

J. B. Les noms mentionnés plus haut, de même que l'émotion que vous mettez à les rappeler, me font croire que vous n'avez pas souvent retrouvé cette qualité d'étudiants.

G. B. Il y avait de tout dans ces produits de collèges classiques : des cancres comme des champions avec lesquels les rapports étaient presque ceux de collègues, comme nous en avions, étudiants, quelques années plus tôt avec nos jeunes professeurs. Je sais votre intention de me demander une comparaison entre cette génération et celle des cégeps. 
Je vais probablement surprendre des lecteurs. La fin des années cinquante et la décennie suivante me semblent avoir été les années creuses dans la qualité étudiante. À se demander parfois si ça valait le coup de continuer! Quand je me laisse aller à des considérations peu optimistes sur les générations, j'entends une clochette d'alarme : «Attention, Bergeron, tu vieillis !» (C'est d'ailleurs un mot dont j'ai horreur. Je le bannis d'ordinaire du langage et le remplace par des expressions comme : «prendre des années, de l'âge » et - pourquoi pas ? - « de la maturité ».)

\section{J. B. Mais les cégépiens ?}

[58]

G. B. La génération des cégeps est arrivée, avec les avantages et les inconvénients d'une formation plus moderne : une vision moins passéiste de la vie et des sociétés, mais aussi, peut-être, un certain manque de fond ou d'information générale pour ne pas parler de culture. J'ai quand même observé de bonnes fournées de cégépiens tant sur le plan de l'écriture que de l'organisation de la pensée. La différence d'avec autrefois, c'est surtout le nombre. Il s'agit d'un tout autre ordre de grandeur quand les effectifs étudiants et professeurs se mettent à décupler. Et puis, l'Université s'est déplacée sur le grand campus, isolé de la ville, à SainteFoy. J'ai parfois la nostalgie du Vieux-Québec. Je profite de chacune de mes courses en ville pour revoir la vieille rue de l'Université, les remparts, le manoir Hébert, la rue St-Louis, la terrasse Dufferin, les rues Saint-Denis et SainteGeneviève, les tavernes où nous prolongions parfois les cours en des discussions animées et chaleureuses ! Le Vieux-Québec sera toujours le lieu privilégié de mon enracinement en ce pays. Les contacts avec les étudiants et collègues sont plis dispersés sur le campus et n'ont plus la même qualité. Toutes les cités universitaires connaissent le même phénomène.

J. B. Une question bizarre, presque farfelue, à poser à un homme qui a trente ans de métier : l'enseignement est-il votre vocation « naturelle " ? 
G. B. Question justifiée puisque, effectivement, je ne me considère pas primordialement comme un homme de l'oral. Mon mode de pensée, de communication et d'influence virtuelle serait plutôt l'écrit. Même si j'ai tendance à être verbomoteur, le message oral n'a pas son maximum de force parce qu'il tend à glisser dans plusieurs directions. Je ne suis pas à l'aise non plus dans les capsules du minutage à la radio ou à la télé. À vouloir faire bref, on risque d'être dense, ce qui amène de l'abstraction qui peut sonner comme de la confusion. Il me semblerait être plus naturellement un homme de l'écrit et, j'ajouterais, du visuel : il me faut voir par écrit pour bien saisir. On discerne aisément chez les hommes [59] publics ceux qui son de l'oral et de l'improvisation, et ceux qui, étant de l'écrit, pensent mieux devant la page blanche et dans le secret du cabinet. Pour ces derniers, la communication orale peut manquer d'impact et ils se trouvent désavantagés.

\section{J. B. Vous trouvez-vous bon professeur?}

G. B. C'est trop gros pour être une question-piège ! Il faudrait plutôt la poser à mes étudiants. Si j'en doute, vous allez me demander pourquoi je continue à exercer ce métier. Je crois être assez bon professeur pour le rester. Je trouve à ce métier une satisfaction générale qui se renouvelle. J'ai de très bons rapports avec mes étudiants qui en retirent sûrement quelque chose. Est-ce que ce rapport bilatéral et oral avec les étudiants me permettrait de m'épanouir dans la totalité de mon être ? La réponse serait « Non » pour les raisons que je viens de dire au sujet de l'oral et de l'écrit.

\section{J. B. Qu'est-ce que vous trouvez de plus pénible dans l'enseignement?}

G.B. Il y a ce côté fastidieux de toujours recommencer l'introduction par le BABA de sa science ! Le professeur a mûri et pourtant, d'année en année, il doit continuer à servir du réchauffé. On doit se refaire dans un effort de didactique et non par un savoir neuf qu'on vient d'acquérir. Ce rôle d'initiateur-introducteur ne m'emballe pas, même sous l'angle du défi, ce qui serait peut-être une indication que je n'ai pas tellement la tripe pédagogique! Je me plais surtout aux niveaux 
supérieurs de la maîtrise et du doctorat où l'on devient animateur dans un savoir à faire, et non plus seulement à distribuer. Une dernière chose : dans la séquence de mai 68, de la génération des cégeps et des nouveaux programmes, je déplore l'esprit de calcul pour des crédits à bon compte. Les étudiants s'attendent à ce que tous passent, et avec au moins un B, la [60] note C leur paraissant peu honorable. Ils viennent parfois négocier leur résultat. Ce côté cafétéria est quelque peu ennuyeux.

\section{J. B. Alors, vous n'êtes pas entièrement heureux dans la carrière universi- taire?}

G. B. J'y suis tellement heureux que je ne me vois pas ailleurs : ni dans la fonction publique, par exemple, même sur le plan international, ni dans le métier chéri des origines, le journalisme. Mais il y a une triple composante dans la carrière universitaire. L'enseignant d'abord, dont la fonction est de transmettre oralement un savoir à des cerveaux en friche. J'aime beaucoup ce contact humain. « Grâce à moi, peut se dire le professeur, ce cerveau pensera peut-être différemment, connaîtra de nouveaux auteurs, n'aura pas peur de s'attaquer à de grandes ceuvres et mettra en question ses idées reçues et ses belles certitudes. » Les discussions en maïeutique, où il s'agit de faire sortir de l'étudiant la réponse qu'il possède mais qu'il ne voit pas, m'apportent une grande satisfaction. De même la direction des travaux où il s'agit de recueillir des données et d'ordonner une complexité.

\section{J. B. Et la deuxième composante?}

G. B. Justement, seconde composante, l'enseignant doit être alimenté par le chercheur. Non pas celui qui « cherche » seulement pour ses cours, mais celui qui réinterprète les connaissances admises et, comme l'on dit, fait reculer les frontières de son domaine. Il est nécessaire, ce chercheur fondamental, chez le théoricien qui doit poser les bases de la connaissance de sa propre science et mettre au point sa propre méthode d' «approche » (qui, je le pense, n'est plus un anglicisme). 
Mais ce professeur est aussi un homme, c'est-à-dire un citoyen. On retrouve finalement le citoyen tourné vers les problèmes du politique, de la Cité dont nous avons longuement parlé lors du premier entretien. [61] Sa responsabilité civique est grande. Par ailleurs, il peut éprouver des difficultés particulières d'insertion dans un milieu social concret auquel il doit signaler les valeurs en jeu et dont il doit promouvoir le progrès. Le journalisme est mon mode personnel d'intervention ; nous en parlerons au prochain entretien.

\section{J. B. Il vous est possible de faire tout cela à la fois ?}

G. B. Le métier le permet. C'est une vie rêvée que celle de l'universitaire. Nous sommes libres d'organiser les 9/10 de notre occupation hebdomadaire, la seule partie imposée étant la présence selon les horaires des cours. Le reste du temps, on peut travailler à la maison. Un minimum de contrainte horaire, sans parler des longues vacances. Maurice Duverger a déjà comparé notre situation à celle des «princes de la Renaissance ». Nous pouvons nous payer le luxe d'être les critiques patentés de la société qui nous confie l'importante fonction de la création et de la transmission d'un savoir. Elle nous légitime en outre d'être ses analystes critiques en nous procurant, par la même occasion, un statut social relativement élevé. Des sondages ont parfois démontré la haute cote d'estime de l'universitaire dans la population, presque à l'égal de celle du médecin ou de l'homme d'affaires qui réussit.

\section{J. B. Les salaires ne sont pas les mêmes, cependant.}

G. B. Surtout durant les années cinquante, avant le règlement des subventions publiques aux universités. Mais même à ce moment, alors que nous vivotions financièrement, nous savions que les choses allaient s'améliorer. Aujourd'hui, le traitement est très honnête. Peut-être est-ce à cette époque de vaches maigres que plusieurs ont développé la manie des revenus d'appoint : recherches subventionnées, commissions, commandites quelconques. Certains ne peuvent plus s'en passer maintenant et les justifient comme un [62] devoir civique ou un mode de 
connaissance complémentaire, ce qui est vrai, en un certain sens. N'empêche que certains universitaires n'auront guère eu d'autres productions que par les commandites des corps publics ou d'entreprises privées.

J. B. Vous passez sous silence une quatrième composante presque inévitable, aux niveaux collégial et universitaire : l'administrateur.

G.B. Je serais une exception. Mon dossier est, ici, sans tache ! J'ai mené une carrière universitaire de plus de trente ans sans jamais présider ni diriger quoi que ce soit. Faut le faire ! Je fuyais les tâches administratives ; elles me fuyaient, devrais-je plutôt dire. Comme je le disais en boutade à un collègue dernièrement : « Mon record, ce n'est pas d'avoir passé toute une carrière sans rien diriger, mais de n'avoir jamais eu l'occasion de refuser une direction ! » Ça fait partie de mon image. On me le pardonne bien à cause de ma productivité de chercheur. Et puis, il y a toujours eu quelqu'un autour de moi, très apte et désireux d'exercer les responsabilités du pouvoir. L'institution n'a donc pas souffert de mon zèle relatif. Appelez cela, si vous voulez, une autre forme de « non branché » : fasciné par les pouvoirs mais peu enclin à les exercer. D'instinct je suis du côté de l'opposant, de celui qui est à l'autre bout de la canalisation des actes du pouvoir et qui dit : n'importe quel pouvoir n'est pas bon ni efficace du fait qu'il est effectif. J'ai le droit, sans faire appel à de grands principes, de discriminer le message tel qu'il m'arrive. Je revendique le droit de juger la marchandise que d'autres me destinent pour mon bien, par eux défini. Je n'ai jamais été d'aucun pouvoir et ça ne m'attire pas d'en exercer un, même pas à l'Université. Comme citoyen, il a fallu que je me trouve une autre fonction. Ç'a été celle de la critique publique, à côté de mon enseignement et de ma recherche fondamentale.

J. B. Il apparaît étonnant qu'après trente ans d'enseignement à l'Université Laval, vous fassiez le saut dans une autre institution. 
G. B. Eh oui ! Je redeviens un jeune professeur qui repart à zéro. D'aîné de la discipline à Laval, incrusté presque dans les murs de l'institution, me voilà devant regagner mes galons ailleurs. Vous parlez d'étonnement. J'ai moi-même été vraiment étonné de cette prise de contact de la direction de l'École nationale d'administration publique (ENAP), une institution de l'Université du Québec qui offre des cours du niveau de la maîtrise à des fonctionnaires de l'État. La direction m'a tenu ce langage : «Il serait naturel que, dans une École où l'on traite finalement de l'État mais toujours par morceaux ou par facettes, quelqu'un vienne nous en parler en sa totalité : d'où ça vient, à quoi ça sert, de quoi c'est fait, l'État. » Il m'a semblé, dès l'abord, que cette demande était faite pour une excellente raison. Par ailleurs, comme sociologue voyant jusque là les choses de l'extérieur, du point de vue du citoyen ou de l'internationaliste, il n'était pas sans intérêt de plonger dans un milieu où étudiants et professeurs échangent sur l'État mais considéré de l'intérieur, sous l'éclairage de l'un ou l'autre des aspects de l'importante fonction administrative. Autrement dit, je complète ma formation, je me recycle pour une vue plus complète de l'État, d'une observation intérieure pour ainsi dire.

C'est l'occasion d'ajuster mon visionnement des choses à l'angle d'optique des praticiens de l'administration publique que j'aurai devant moi, plus âgés et mieux motivés, sélectionnés en raison du poste qu'ils occupent, de leurs qualités particulières et de leurs chances d'avenir. Maintenant que l'ENAP a assuré sa crédibilité, elle est prête pour un second souffle et on me fait l'honneur de m'y associer. C'est vraiment très séduisant, d'autant que l'esprit qui y règne m'apparaît très cordial et d'une belle dynamique institutionnelle.

\section{J. B. On ne peut pas parler de rupture de carrière ?}

G. B. De continuité plutôt. Il n'y a pas de différence radicale entre Laval et l'ENAP ; mais la seconde m'y offre de meilleures conditions intellectuelles de travail. J'ai en chantier un livre qui est peut-être la plus risquée de mes entreprises jusqu'à maintenant. Il s'agit d'y [64] confronter mes schématisations théoriques sur l'État - toujours lui - à l'étude d'une société concrète, la nôtre, qui a connu d'extraordinaires mutations depuis une vingtaine d'années. C'est, un projet assez emballant : pour la première fois dans ma carrière, je joindrais, en un seul ouvra- 
ge, mes deux visionnements du politique, le théorique généralisant et le conjoncturel particularisant. Et il se trouve que cela se produit à une époque critique où, par étapes de plus en plus pressantes, se pose en totalité la question politique du destin collectif des Québécois. Autrement dit, cet ouvrage est un rendez-vous entre la théorie et l'histoire, entre l'État et la société et, finalement, entre l'analyste et le citoyen dans le même homme. D'une autre façon, c'est encore une tentative pour valider ma propre théorie, bien que personne ne me presse pour m'imposer ce test. Si j'étais plus prudent, je m'en abstiendrais en espérant que d'autres le fassent à ma place.

\section{J.B. Et si vous aviez été quelque peu plus « entrepreneur " universitaire, cela se serait peut-être produit auparavant?}

G. B. Il aurait été conforme à un certain «profil de carrière » que je lance, à point nommé, quelque chose comme un centre de théorie politique à Laval. Dans notre département, il y avait un nombre proportionnellement considérable de collègues intéressés à des travaux théoriques. Au point que Vincent Lemieux, luimême engagé profondément dans ce type de recherches, concluait, dans une étude bilan sur la question, que les politologues du Québec n'avaient pas à nourrir de complexes à l'échelle de la francophonie internationale sans exclure la France. Il n'y a pas que mon manque du sens de l'entrepreneurship en la question. Pour dire toute la vérité, j'aurais eu une répugnance à sembler me mettre en position de faire la promotion de mes travaux dans le genre ou encore de défendre mon « terrain théorique », selon une expression qui doit être entendue avec une nuance ironique... 
[65]

GÉRARD BERGERON.

de l'autre côté de l'action.

$3^{\mathrm{e}}$ entretien

LE THÉORICIEN

«... par une espèce d'instinct
de conservation »

$\underline{\text { Retour à la table des matières }}$ 


\section{J. B. Voulez-vous prouver que la théorie peut servir à quelque chose par cette recherche en cours sur la gouverne politique, ou le fonctionnement de l'État, au Québec?}

G. B. Si vous voulez, même si les preuves ne manquent déjà pas dans l'histoire de la discipline. La théorie est une mise en place ordonnée des éléments majeurs d'un ordre de phénomènes qu'elle qualifie, par des concepts et propositions appropriés, afin de rendre intelligible un réel multiple et diffus. Par son essai de visionnement d'une dynamique globale, elle part en deçà et va au-delà des points de vue analytiques, que nous faisons tous dans l'étude de conjonctures, et dont l'exemple le plus facile est le commentaire éditorial. La théorie élargit le champ de connaissance, le stimule, peut en engendrer de nouveaux. Elle sert au moins à cela, qui n'est pas négligeable. Elle est aussi, secondement, l'indice de maturation d'une science. C'est quand même intéressant de travailler à l'édification d'une des sciences humaines, a son theory building. Ces sciences sont si bavardes, si anarchistes, si primitives. Le théoricien se situe juste en-deça de l'épistémologue qui oeuvre à un niveau encore supérieur parce qu'il s'intéresse à la connaissance en tant que telle.

\section{J. B. Les théoriciens ont la réputation de dire de façon compliquée des choses simples...}

G. B. ... et de trop simplifier les choses compliquées puisqu'ils ramènent à l'unité les phénomènes complexes. À priori, nous sommes perdants des deux bords ! Le reproche tient principalement au mode de communication parfois difficile de la théorie. Quant au jargon qu'il n'est pas toujours possible d'éviter, je vous ferai remarquer qu'on l'accepte facilement pour les sciences de la nature, qu'on dit aussi « pures » ou « exactes ». Pourquoi pas pour les sciences sociales ? Pourquoi 
pas pour la science politique, en particulier, si c'est nécessaire, mais vraiment nécessaire, à sa scientificité présumée ou prétendue ?

\section{J. B. Comment devient-on théoricien ?}

G. B. Dans mon cas, ç'a été sans le savoir et sans le vouloir, à l'occasion d'un sujet de thèse de doctorat qui nécessitait un cadre théorique inexistant pour être mené à bon port. Je vous en ai parlé à l'entretien précédent. Mais c'est peut-être aussi en voulant mettre de l'ordre dans la formation du produit hybride que j'étais : Français d'Amérique, bilingue, faisant des études sociales à l'américaine en même temps qu'à l'européenne. J'éprouvais le besoin aigu de m'y retrouver. Peut-être que si j'avais été un Nord-Américain non francophone, je n'aurais pas dit la même chose ou même je n'aurais pas eu l'idée d'embarquer dans une pareille galère.

Ce caractère « hybride » provenait aussi d'un autre facteur, celui de mes premières études. Cette philosophie du droit public, du droit de l'État, je l'ai abordée par le prisme de l'observation sociologique qui est tout à l'opposé puisqu'elle s'occupe primordialement des représentations et comportements collectifs et non du principe juridique lui-même. Plutôt, le principe est à retrouver dans les attitudes et les conduites. C'est donc différent et du juriste et du philosophe qui, tous deux, déduisent et du sociologue empirique qui l'amène d'abord à induire à partir de la réalité sociale. Mais j'avais à devenir un peu tout cela à la fois, dans une certaine unité qu'il a fallu trouver et que je maintiens toujours en ne cédant pas exclusivement à [69] l'un ou l'autre de ces points de vue. Je suis devenu théoricien, pourrais-je dire, par une espèce d'instinct de conservation pour ce que je voulais réussir à faire. Cela dit, je ne suis pas sûr qu'il fallait répondre à une telle question, tant il m'a fallu simplifier la réponse (...).

J.B. Une fois théoricien, non pas par erreur, mais presque par inadvertance, et sans vous rendre très bien compte de ce que cela allait impliquer par la suite, il a bien fallu justifier votre démarche particulière envers d'autres démarches possibles ou déjà pratiquées, c'est-à-dire préciser une marque de fabrique particulière. Ce qui s'appellera votre « fonctionnalisme ». 
G. B. Oui, mais en un sens très particulier qui est celui de la « nature des choses » dont parlait Montesquieu et de la « division du travail » dont parlera plus tard Durkheim principalement : c'est un fonctionnalisme du fonctionnement et de toute espèce de « fonctions-processus » du politique, en tant que tel et à l'intérieur des grandes unités de base que sont devenus les États modernes. C'est une très longue tradition qui remonte à Aristote. D'autre part, il y a un fonctionnalisme, ou plutôt un structuro-fonctionnalisme à la moderne, qui, tout en se réclamant plus ou moins des mêmes grands ancêtres, est beaucoup plus abstrait et technique. Dérivant de l'idée très large, et à vrai dire accommodante, de système, il se fait fort en particulier d'appliquer à l'étude du politique les principes de la théorie des systèmes et même certaines techniques hautement valorisées aujourd'hui en analyse des systèmes. Cette approche, avec les apports assez sophistiqués de la cybernétique, de la théorie de l'information, de l'analyse par input-output, a fait recette ces dernières quinze ou vingt années en science politique, après avoir connu une vogue très grande en sociologie sous l'impulsion générale de Talcott Parsons (dont l'oeuvre colossale et complexe a été rendue plus abordable, avec des simplifications et dissidences nécessaires, par quelques disciples dont Guy Rocher, qui fut son étudiant à Harvard). Ici, permettez-moi de ne pas [70] emprunter un chemin de traverse pour distinguer divers fonctionnalismes en science politique (...).

\section{J. B. Je crois voir que votre « fonctionnalisme » est plutôt le traditionnel, celui du fonctionnement et de la nature des choses, que ce fonctionnement à la moderne que vous venez de rappeler.}

G. B. Exactement, bien qu'il y ait une commune aspiration entre les deux : voir d'abord « comment ça marche », « comment ça tient ensemble ». Mais dans le second cas, l'aspect méthodes et techniques prend une place peut-être démesurée en rapport à la substance ou à la trame de la théorie elle-même qui doit surtout rendre compte des processus fondamentaux à divers niveaux d'analyse.

\section{J.B. En somme, vous êtes mi-classique, mi-moderne.}


G.B. Cela pourrait se dire ainsi. Le regretté Pierre Duclos, un de mes tout premiers critiques en France, estimait que ma théorie était «novatrice pour les classiques et classique pour les novateurs ». La conséquence en est que je me retrouve plutôt seul, sinon isolé, à peu près de même façon qu'en mon rôle de critique des problèmes politiques immédiats du milieu, du moins tel que je le conçois et le pratique.

\section{J.B. Mais il y a aussi les marxistes?}

G.B. Et spécialement ceux de la branche « néo », non seulement parce qu'ils sont nouveaux et jeunes, mais. parce qu'ils attachent maintenant une importance capitale au phénomène de l'État et de la politique comme activité, ce qui était vraiment un point faible et négligé de la doctrine marxiste. De jeunes théoriciens en France et en divers autres pays européens et, ces dernières années, aux ÉtatsUnis, ont voulu animer et [71] réactualiser le marxisme en le politifiant pour ainsi dire. Une partie très importante de nos politologues et sociologues de la jeune génération travaillent selon ces lignes et certains ont produit des travaux d'importance. Après une extraordinaire effervescence d'une dizaine d'années, il semblerait toutefois que s'atténuent quelque peu l'attrait et la popularité de ce courant qui s'est fait assez envahissant en certains départements universitaires, d'autant qu'il était porté par plusieurs des plus doués parmi nos jeunes chercheurs et enseignants. Voilà un secteur où le Québec n'a guère pris de retard...

\section{J.B. Entre Marx et Parsons, il y a aussi d'autres voies?}

G.B. S'il fallait continuer ces «patronages » assez encombrants, il faudrait relever, moins une filiation au sens strict, que divers apparentements : Montesquieu, Tocqueville, Weber, quatre ou cinq continuateurs contemporains en sociologie historique. Cela forme une série « impressionnante », mais assez peu intimidante, puisqu'une théorie générale de l'État d'aujourd'hui reste toujours à faire... 


\section{J.B. ...pour laquelle vous proposez vos propres perspectives}

G.B. ... et qui ne se livreraient guère, vous le pensez bien, en quelques propositions carrées à l'occasion de ces propos qui vont vite, vite !

\section{J.B. On peut toujours référer à vos livres et à vos articles.}

G.B. Bien sûr, c'est pour cela que je les ai écrits : pour d'abord mettre de l'ordre dans mes idées et les communiquer ensuite au public de la discipline. Mais même alors, comme je travaille en dehors ou en deçà des deux paradigmes dominants, « fonctionnaliste »ou « systémiste » et « néo-marxiste », et en une plus [72] lointaine tradition que ces formidables machines de pensée et d'analyse, ma propre recherche n'est pas aisément identifiable, n'est pas portée favorablement par un des grands courants du jour. En bref, mon effort apparaît d'une grande étrangeté, qui déconcerte, du fait qu'on ne puisse pas aisément le classer. C'est d'ailleurs assez bizarre cette sensation d'isolement dans le fait d'oeuvrer dans le prolongement d'une tradition on ne peut plus « classique », tout en étant très ouvert à divers apports d'un éclectisme raisonné.

\section{J. B. N'y a-t-il pas d'autres raisons? Nous parlions de jargon tout à l'heure.}

G. B. En effet, je m'en suis expliqué franchement dernièrement. Mon langage ou mon jargon n'est pas plus « particulier » que celui qui est en usage dans d'autres discours théoriques qu'on accepte aisément parce qu'on a eu le temps de s'y habituer ${ }^{3}$. Ce qui est beaucoup plus important dans ma tentative, ce sont quel-

3 Dans un article intitulé « Comment peut-on être Persan ? » et qui est la contribution de Gérard Bergeron aux Mélanges en l'honneur de Jean-Charles Falardeau, sous la direction de Fernand Dumont et Yves Martin, à paraître aux 
ques postulats en lesquels je crois fermement : $1^{\circ}$ d'abord que, pour faire une théorie de l'État, il n'est pas nécessaire d'oublier ce qu'on sait déjà d'une science politique traditionnelle en accueillant, en les réincorporant, des apports très pertinents des plus récentes sciences sociales ; $2^{\circ}$ qu'il faut s'appliquer d'abord à une théorie du fonctionnement en la distinguant le plus nettement possible d'une théorie du changement, ne serait-ce que pour voir avec clarté ce qui a changé, change, peut ou doit changer ; $3^{\circ}$ qu'il faut se méfier des facilités de départ - qui compliquent bien des choses dans la suite - en posant la question dans les rapports entre les deux systèmes, le politique et le social (ou le socio-économique), qui communiquent entre eux par toutes sortes d'interactions et de rétroactions.

Ce que je propose à la place, c'est un mode de visionnement unitaire où État et Société sont un dans un Nous qu'ils forment ensemble dans la réalité, mais posé en trois niveaux à la verticale, que j'appelle respectivement les niveaux du régime, de la gouverne et de la politie (ou société politique). Mais arrêtons là, [73] si vous voulez. Le peu que je pourrais en dire, ici, au-delà de ces positions de départ, n'aurait qu'une faible valeur informative et brouillerait la relative clarté de ces propos forcément très généraux et qui paraissent, de ce fait, suffisamment abstraits.

J.B. Si vous aviez écrit en américain plutôt qu'en français et à partir d'une autre base de départ que Québec...

G. B. Je m'interdis d'instinct à lire ma petite histoire par « Si... », autant que la grande. «Si..., si... »; personne ne peut savoir. Alors !

J.B. C'est par cette oeuvre théorique surtout que vous êtes connu à l'extérieur de nos frontières. Vous n'êtes pas nombreux, au Québec à jouir d'une reconnaissance extérieure dans le domaine des sciences de l'homme.

Presses de l'Université Laval sous le titre probable de Représentation sociale et imaginaire collectif. 
G.B. Du seul fait que je publie mes ouvrages de théorie politique en France, j'ai accès à l'ensemble de la francophonie et à tous les pays, nombreux, où le français est une langue de recherches universitaires. Pour publier en France, il faut présenter une production internationale justement. Les études sur le Québec ou le Canada nous concernent principalement et n'intéressent, à l'extérieur, que les spécialistes rarissimes qui s'occupent de nous. Alors que les questions de méthode, de théorie, d'épistémologie débordent les frontières, sont les mêmes partout, s'exportent.

J.B. Vos ouvrages doivent bien être recevables, sans quoi ils seraient bloqués au niveau des comités de lecture ou torpillés par la critique une fois publiés.

G. B. Il faut courir sa chance sur le marché libre des éditeurs étrangers et s'exposer aux critiques des spécialistes qui ne prendront pas en compte la nationalité de l'auteur ni une politique de promotion de la [74] recherche. En ce sens-là, on n'est pas très armé pour percer le mur de l'édition parisienne.

J.B. Consacré théoricien, considéré par des sommités de la science politique comme Raymond Aron et Bertrand de Jouvenel, objet de plusieurs thèses : voilà qui accrédite un certain magistère. Faisant un article sur vous, il y a quelques années, $j$ 'avais vu dans votre correspondance professionnelle des entêtes où on vous servait du « maître ». Comment vivez-vous cela ?

G. B. Je ne me suis jamais personnellement reconnu de «maîtres » et je ne marcherais pas à coups de « Maître » : ça n'entre pas dans mes schèmes de pensée puisqu'il y a toujours un élément d'abdication dans ces procédés de révérence. Ce n'est pas complètement faux, non plus, puisque des chercheurs travaillent selon vos pistes ou vous adoptent comme un de leurs référents théoriques, ne serait-ce que pour porter des jugements de disqualification! Dans les cas que vous signalez, il faut entendre une nuance d'affection et d'ironie. Cette notion de maître à penser me semble plutôt désuète et réfère à des critères d'école utiles pour des 
classements rapides. Ici Groulx en a été le prototype de ce siècle en même temps que, probablement, le dernier spécimen. Notion pernicieuse aussi, puisqu'elle indique une adhésion adulatoire, souvent dépourvue de discrimination critique, très reliée aux vogues d'un moment. Rien encore de tel dans notre milieu ; et c'est heureux ! Ce qu'il y a dans ce pays, ce sont des voix pas très nombreuses qui, à divers niveaux de langage scientifique, ont gagné une certaine écoute à l'instar de contributions venant d'ailleurs. Qui prétendrait aujourd'hui, dans une petite société intellectuelle comme la nôtre, à cette espèce de demi-mythologie du maître à penser d'une génération, serait amené à adopter des comportements risibles et pas du tout crédibles, bref à prendre des allures de « petit maître » !

\section{J.B. Ne me dites pas que vous êtes indifférent à ces [75] manifestations de} fidélité d'étudiants se proclamant disciples !

G.B. Bien sûr que non. Cette gentillesse, d'ailleurs teintée d'ironie, je le répète, fait prendre conscience d'avoir réussi quelque peu après tant d'années investies dans le domaine aléatoire de la théorie. Elle incite au moins à la responsabilité de ne pas lâcher. De pouvoir participer à l'élaboration de sa discipline, que mettent en opération nombre de chercheurs de plusieurs pays, déplie et ouvre sur l'univers scientifique son « petit Québécois » ! Par la langue française, deuxième langue de communication scientifique de la planète, langue de civilisation depuis plusieurs siècles, on a accès au même marché potentiel qu'un chercheur français. On peut trouver preneurs et correspondants sur une scène plus large que le Québec, d'autant plus qu'il se publie fort peu en français dans ce domaine de l'élaboration théorique.

\section{J.B. N'empêche que cette réputation ouvre des portes à l'étranger.}

G.B. Il ne faudrait pas en exagérer l'importance. Vous arrivez dans une université étrangère : on vous connaît pour vous avoir lu et critiqué. On vous présente comme celui qui représente la science politique du Québec sous telle dimension. Les portes s'ouvrent, il est vrai, mais on ne déroule pas les tapis rouges ! Une 
fois sur place, vous devez vous dédouaner tout seul. Il faut être capable de défendre vos travaux devant des groupes d'universitaires, à des colloques. Il faut surtout que le livre suivant soit à la hauteur des attentes que vous avez suscitées, à moins qu'on ne vous ait déjà oublié dans l'intervalle ! Bref, c'est à gagner tout le temps. Je n'éprouve aucune aliénation de Québécois à devoir le faire. J'aurais pu faire la même oeuvre théorique n'importe où dans le monde. Dans cette émission radiophonique, dont nous avons déjà parlé, avec Roger Nadeau et Fernand Dumont, Fernand disait : «L'important n'est pas de parler d'ici, mais de [76] parler à partir d'ici ». Je parle à partir d'ici. Si ça se rend ailleurs, tant mieux !

\section{J.B. Mais, dans l'autre partie de votre oeuvre, vous parlez d'ici et surtout aux gens d'ici.}

G.B. Inversement, quand je parle d'ici aux gens d'ici, dans Le Devoir par exemple, lu par les mille personnalités importantes de ce pays, cela peut avoir un retentissement disproportionné par rapport au temps que vous y mettez et à la minceur du message. Mais alors, on prend un certain risque en jouant cette réputation dans un simple article de journal ou dans une brève apparition à la radio-télé où l'on fait, finalement, du subjectivisme impressionniste sur l'actualité de la journée, comme tout le monde. L'attraction de ces succès par trop faciles risque de faire dévier ou même de gâcher des carrières universitaires de chercheurs.

La vie de théorisateur - je préfère ce mot à théoricien, il fait plus artisan au jour le jour, moins « arrivé » - n'est pas facile. Il y a surtout ce terrible doute de vous-même, puis la portée aléatoire de l'investissement de vie que vous mettez dans ces travaux longs et austères, alors que vous pourriez faire autre chose de plus immédiatement «rentable » au plan intellectuel. Enfin, des gens fort bien intentionnés vous rappellent à l'occasion que d'autres responsabilités vous obligent aussi dans l'administration universitaire ou sur la place publique. À cela vous opposez presque honteusement la nécessité de la tour d'ivoire, un argument peu convaincant de prime abord, taxé d'abstrait et d'égoïste par vos interlocuteurs. Et pourtant la théorie n'est pas irréelle, déconnectée. Elle est la clé d'intelligibilité d'un réel complexe. C'est en ce sens qu'on a pu dire que rien n'est plus pratique 
qu'une bonne théorie. Elle seule permet une lecture bien plus profonde de ce réel que ceux qui ont le nez dessus comme, singulièrement, les hommes d'action.

J.B. Votre première éclipse journalistique a coïncidé avec [77] les prémisses de la Révolution tranquille. Sous Duplessis, vous appeliez ces changements. Ils se produisent, vous n'êtes plus là pour les analyser ou aider à les redéfinir. Comment expliquer ce silence?

G.B. Cela fut tiraillant. J'avais décidé de fermer le robinet de mes interventions journalistiques en 1958 pour m'engager à fond dans le défi de ce qui allait être Fonctionnement de l'État. Quoi qu'il advienne. Et il en est arrivé des choses, à commencer par la mort de Duplessis en septembre 1959, les Cent Jours de Sauvé, l'intérim de Barrette, les élections de juin 1960 et tout le reste. Mais je ne peux pas en même temps besogner dans cette tour d'ivoire et descendre sur la place publique pour des analyses de conjonctures, par définition toujours changeantes. Il reste à procéder par alternance. Après un gros coup dans le travail théorique pendant plusieurs années, on sent le besoin d'en sortir, de s'en désintoxiquer. On y arrive d'autant mieux que ce moment coïncide avec une inquiétude qui peut s'emparer du citoyen sur ce qui se passe ou devrait pouvoir se passer ici.

\section{J.B. La fin de La Gouverne politique a «heureusement » coïncidé avec l'automne 1976.}

G.B. J'aurais eu besoin de douze autres mois pour polir et consolider l'ouvrage. J'avais décidé d'y mettre le point final et d'aller le porter chez un éditeur parisien au printemps 1976. Je ne vivais que pour ce livre depuis cinq ou six ans; c'était trop psychologiquement. Mais surtout, la conscience civique-honteuse ? s'éveillait chez moi. On sentait la fin des années Bourassa; la montée du P.Q. était marquante, les sondages le plaçaient à la porte du pouvoir ; les rumeurs d'élections anticipées circulaient. Je voulais être aux premières loges. Je l'ai été depuis : mes écrits journalistiques de 1976 à 1981 forment la matière de quatre livres. C'est la première fois que j'ai une aussi longue collaboration de ce type. 
N'eussent été la tenue tardive du référendum [78] et l'offensive constitutionnelle de Trudeau, j'aurais quitté la place publique plus tôt.

J.B. Vous parliez tout à l'heure de la mise en branle d'un projet dans lequel vous appliqueriez vos schèmes analytiques à la société québécoise. Cette plongée théorique signifie-t-elle un autre retour à la tour d'ivoire ?

G.B. Pas à ce point. Il me faudra suivre de très près les dernières phases d'évolution de notre société, objet de mon étude. Mais pour cela il n'est pas nécessaire de multiplier les chroniques d'actualité. J'espère continuer à être présent sur les deux plans, mais en des alternances plus courtes. Si j'avais concentré mes efforts sur la recherche fondamentale, peut-être aurais-je complété le cycle de mes travaux théoriques, mais cela aurait été trop me refréner dans l'une de mes composantes d'homme, celle du citoyen.

J.B. Votre formalisation théorique est-elle une coquetterie de carrière ou aide-t-elle vraiment le journaliste-analyste dans ses incursions dans l'actualité ?

G.B. La question terrible entre toutes ! Quand on fait de la théorie, on aspire à faire de la science ; quand on fait du journalisme, on fait de la critique immédiate sur une actualité contingente. Ce n'est pas contradictoire, mais il s'agit de deux niveaux différents de compréhension. Un même individu peut écrire comme analyste en faisant oublier qu'il est aussi théoricien. Sans sortir toute la machinerie théorique et l'appareil conceptuel, il peut discerner, dans l'actualité découpée en quotidien, ce qui échappe d'ordinaire au non théoricien, soit les dynamiques d'ensemble et les nécessités du système. À l'inverse, il risque le danger de trop, ou de trop vite, systématiser. La subtilité consisterait à mettre en veilleuse ce côté théoricien le plus possible. Pour être plus lisible d'abord... 
J.B. Pourtant vos écrits journalistiques ont une densité qui laissent deviner le conceptualisateur.

G.B. Vous êtes meilleur juge que moi. En tout cas, j'essaie de l'atténuer le plus possible. Ou de renverser la priorité : ce matin, me dis-je, c'est le journaliste qui parle prioritairement. Mais je sais bien que mon approche aux problèmes reste marquée par un certain fond d'équipement théorique, même quand je n'ai pas à le dissimuler dans un texte bref et ponctuel.

\section{J.B. Votre « équipement théorique » vous sert donc finalement ?}

G.B. Probablement. Par toutes sortes de suggestions. Je n'aurais pas écrit la même oeuvre analytique sans mon visionnement du politique global. Et l'analyste de conjonctures s'informe par les média, par les journaux (j'en lis et en découpe énormément). L'information que j'y recueille ressemble à celle d'une salle de rédaction. Il faut qu'à point nommé je sois capable de sortir les pièces importantes d'un dossier sans l'aide de recherchistes, et d'écrire en deux ou trois heures, comme un journaliste, mais sans savoir, comme celui-ci, s'il paraîtra le lendemain.

Ce « coffre à outils » théorique, je l'ai donc garni au jour le jour par la lecture des journaux le soir et en essayant de m'y reconnaître dans ces problèmes quotidiens qui s'entremêlent. Il est possible de prendre une page de nouvelles internationales ou de politique canadienne et de traduire cela, d'instinct, en langage théorique ; on peut suivre le chemin inverse. Je le fais devant mes étudiants pour illus-

trer que la théorie part du réel et y retourne : c'est normal puisqu'elle en est la clé d'intelligibilité. Une fois qu'ils sont assouplis à mon appareil analytique, je leur demande de faire la même chose. Un de mes anciens étudiants, maintenant un collègue en journalisme et communication à Laval, Jacques Rivet, que mes travaux théoriques avaient attiré et qui est resté un critique très stimulant, me signalant souvent des faiblesses et des vides dans [80] mon schéma, donne comme travaux à ses étudiants sa théorie «bergeronrivetisée » (comme il le dit avec humour) appliquée à nos journaux. J'ai pris connaissance de travaux de ses étudiants qui étaient d'une excellente qualité analytique et technique. 


\section{J.B. Ainsi, la théorie d'apparence abstraite a un côté pratique.}

G.B. Dans le cas cité, cela pour vous dire que le travail peut s'effectuer dans les deux sens : de l'analyse quotidienne à la théorie et de la théorie à l'analyse quotidienne. Et que c'est communicable. Ma théorie sert à d'autres, par exemple aussi à Lionel Ouellet, spécialiste en théorie administrative à l'ENAP, qui corrige, élague et prolonge mes propositions théoriques trop générales pour l'examen de son domaine propre. Une théorie n'est pas d'abord une limite, une camisole de force. C'est plutôt un point de départ, un tremplin. Elle n'est jamais aussi utile que lorsqu'on l'oublie après s'en être servi pour interroger la réalité. Le tout, c'est d'avoir « la tête théorique »...

\section{J.B. Vos étudiants ne doivent pas être tous du calibre de ceux que vous} venez de mentionner?

G.B. Mon enseignement a pu « contaminer » quelques-unes de nos têtes théoriciennes actuelles, même si ces gens ne travaillent pas du tout selon mes pistes, ou oeuvrent à un autre niveau de théorie. Un des plus connus, un théoricien de grande originalité et de première force, un de mes tout premiers critiques aussi comme étudiant, c'est Vincent Lemieux, qui travaille selon une ligne dite « formaliste », beaucoup plus près des relations de pouvoir et d'influence, et qui utilise l'outil mathématique et cybernétique. Il ne publie pas à l'étranger mais y est connu de gens de sa spécialité. J'ai donné le goût de la théorie à d'autres aussi, que je ne vais pas tous vous nommer et auxquels il est resté quelque chose, fût-ce la détermination de [81] cesser d'en faire ! Ça sert au moins à cela... Car, il n'y a pas de science ou de connaissance que dans et par « la théorie » (au sens strict et explicite).

J.B. Est-ce que le fait d'être théorisateur rend plus apte à juger l'écheveau politique d'une société ? En d'autres mots, vous estimez-vous plus crédible qu'un éditorialiste de métier ? 
G.B. Oh là là ! Dans quoi êtes-vous en train de m'embarquer ? C'est une question énorme, d'incongruité ou d'ingénuité. Pensez bien que je n'y répondrai pas. Je ne me substitue pas à la critique. Si quelqu'un le disait, ce serait peut-être vrai : si quelqu'un d'autre en doutait ou y contredisait, ce serait probablement non moins exact.

\section{J. B. Qu'en pensez-vous personnellement, sans fausse modestie ?}

G.B. Ce n'est pas un problème de modestie - je ne pense pas être modeste, ni faux - mais un problème de véracité. Quand je réagis en tant que citoyen, j'y vais avec la totalité de mon être dont le théoricien n'est pas absent. Mais j'y vais aussi avec toute ma subjectivité d'un individu en particulier. Et sur ce plan, j'ai les mêmes préférences, les mêmes instincts, les mêmes allergies que n'importe quel intellectuel de mon espèce. Si je pensais disposer d'une sagesse particulière, pensez bien que je ne l'admettrais pas!

\section{J. B. Votre réponse est négative : le théorisateur n'est pas nécessairement plus apte à...}

G.B. « Nécessairement », non. Si vous aviez dit simplement « apte »la réponse pourrait être : «Ce n'est pas nécessaire, mais c'est à voir... » Il faut au moins accepter, je crois, une présomption d'aptitude. Pas sur n'importe [82] quel sujet, mais quand le débat se rattache à l'un ou l'autre des aspects que couvre la recherche du théoricien dans sa spécialité. Mais nous voilà renvoyés au problème, insoluble, de l'expertise et de la contre-expertise ! À l'expert ! s'oppose le contreexpert !!. Qui les départagera, ultimement, en nos domaines ? L'Histoire, mais c'est longtemps après, and we all be dead - selon la formule de Keynes.

J.B. Mais il y a tout de même des exposés qui, sous certaines signatures, ont plus d'autorité que d'autres? 
G.B. Oui, sur le plan de la « crédibilité », justement, où vous avez posé votre question. En français, "crédibilité » veut dire ce qui rend une chose digne de croyance. Sous l'influence de l'anglais probablement, nous avons étendu le sens du mot aux personnes dignes de croyance. Notez qu'il s'agit toujours de « croyance ", soit de ce qui n'est pas absolument probant, ni qui peut être complètement démontré. Donc, la crédibilité repose sur autre chose que la seule validité de ce qui est avancé. La position sociale, les règles de comportement moral que s'impose l'intervenant en matière sociale, en politique sont peut-être la partie la plus solide de cette base de crédibilité. C'est à longueur de toute une carrière que cela se fonde ou se forge. Cela relève autant du style de vie morale que de la force intellectuelle.

\section{J.B. Il vous arrive tout de même de prendre vos risques lorsque vous lan-} cez dans le public des projets assez hardis où perce l'imagination en même temps que la rigueur d'exposition du théoricien.

G.B. Oui, je prends parfois de tels risques. Quand je me livre à des études de conjonctures politiques, je m'efforce de présenter des analyses serrées, réalistes des actions en cours des hommes et groupements politiques. Je ne me retiens pas toujours de leur rendre des points en leur " pragmatisme » (célébré par [83] euxmêmes) : disons que c'est une petite coquetterie, teintée d'un brin de sadisme, qu'un théoricien en tour d'ivoire leur administre ces petites leçons de réalisme et de sens pratique. Pour que ces critiques portent, il faut se placer dans la perspective même de leur programme d'action (ou de ce qui en tient lieu !) et n'en pas contester le principe ou, tout au moins, le mettre entre parenthèses. Mais ce ne sont pas là mes vrais risques de théoricien. C'est plutôt lorsque j'explore le champ des possibles dans des évolutions désirables dans le domaine de réarrangements institutionnels en particulier. 


\section{J.B. Votre fameux plan d'un nouveau Commonwealth canadien ${ }^{4}$, par} exemple, à mi-chemin entre les divers projets de fédéralisme renouvelé et celui de la souveraineté-association ?

G.B. Ou celui des mécanismes de réforme constitutionnelle ${ }^{5}$, que j'ai également colporté ici et là, pour élargir et quelque peu stimuler le débat, tournant en rond ou ne considérant que les positions antinomiques. Sans me réfugier dans l'imaginaire pur, porte d'entrée naturelle pour l'utopique, il m'est arrivé de m'affranchir des contraintes qui freinent l'invention pour réétudier le réel en vue de discerner des potentiels d'évolution, moyennant telles conditions à transformer préalablement.

Il faut au moins porter autant d'importance aux règles de transformation qu'au résultat escompté ou désiré de la transformation. Ce qui me frappe dans la pensée politique en action de ces superbes «réalistes » que sont les hommes politiques, c'est qu'ils ne font pas le point, intermédiaire, entre ces visionnements de myopie et ces vues de presbytie. Ils sont faibles sur le plan des moyens. Ils discernent mal $\mathrm{B}$ lorsqu'il s'agit de passer de $\mathrm{A}$ à $\mathrm{C}$. Qui leur parlera de $\mathrm{B}$, sinon les gens de l'espèce rare qui peuvent le faire ? Ce n'est jamais complètement perdu, puisque de tels exercices peuvent du moins servir pour des comparaisons qu'on ne pourrait pas faire autrement ${ }^{6}$.

4 «Une solution 'super-fédéraliste' à la crise canadienne : un Canadian Commonwealth Canadien », The Canadian Bar Review/Revue du Barreau canadien, décembre 1979.

5 «Il faut faire quelque chose, mais COMMENT ? " Dans Mécanismes pour une nouvelle constitution, ouvrage en collaboration avec Edmond Orban, Edward McWhinney et Gérald A. Beaudoin, Ottawa, Éditions de l'Université d'Ottawa, 1981.

6 Ainsi, en rapport au modèle indiqué à la note (2), « Lecture du Livre blanc et du Livre beige selon une perspective 'superfédéraliste' », Canadian Public Policy/Analyse des politiques, VI, nº 3, été 1980. 


\section{J.B. Vous êtes aussi internationaliste. En tant qu'historien de la guerre froide, comment la vivez-vous?}

G.B. Ayant fait le choix d'une carrière d'internationaliste au moment de la Seconde Guerre mondiale et des horribles champignons d'Hiroshima et de Nagasaki, je n'ai jamais pu me détacher de cette question ultime de la survie de l'humanité dans la paradoxale assurance de l'équilibre de la terreur. Cette question angoissante a imprégné ma sensibilité profonde, d'autant plus qu'elle était devenue le leitmotiv de la littérature et de tous les forums internationaux d'après-guerre. Pour la première fois dans l'histoire, l'humanité avait construit des engins qui pouvaient aseptiser la planète entière en deux heures. Il y suffirait d'une fraction de l'arsenal d'un des deux Grands. Le genre humain a les moyens de son suicide. Nous sommes à la merci non seulement des folies dont sont capables les hommes de pouvoir, mais d'une simple erreur de détection technique. Voyez la récente affaire des bombes à neutrons dont le président Reagan a ordonné la production et les protestations de plus en plus importantes des mouvements pacifistes et anti-nucléaires. La troisième guerre mondiale est désormais impensable, mais il faut malgré tout y penser. En période de crise internationale, les chefs d'État américain et soviétique, le secrétaire général de l'ONU, n'ont rien moins que la responsabilité de la survie de l'espèce humaine entre leurs mains.

De ce que j'en enseigne, ou de ce que j'en écris, il en reste quelque chose dans la personnalité. J'ai retrouvé une inquiétude similaire dans des oeuvres marquantes de notre époque. Mais je ne l'ai jamais trouvée avec autant d'intensité et d'immédiateté que dans le splendide film d'Alain Resnais, Hiroshima, mon amour. Un film qui posait tout le problème du dépassement de notre qualité d'homme et de femme en épousant et repoussant ce cataclysme au bord duquel nous vivons constamment. « Tu n'as rien vu à Hiroshima... », répète le Japonais à son amante française d'une nuit. Heureusement que, pour un relatif équilibre de nos psychismes profonds, nous pouvons, nous devons même penser à autre chose et nous [85] activer à du plus immédiat et du moins angoissant : nos petits tracas personnels, nos bobos quotidiens et nos préoccupations économiques et politiques locales. C'est cette bienheureuse inconscience qui nous empêche de sombrer dans la psychose de la survie de l'humanité. 


\section{J.B. Mais il y a aussi le fait du sous-développement.}

G.B. Hélas ! Et c'est encore moins drôle puisque c'est du réel et non pas seulement du menaçant. C'est du réel, les zones de sous-développement, le Tiersmonde, le présumé dialogue Nord-Sud. Une moitié de l'humanité - certaines statistiques disent même les deux tiers - vit en deçà du seuil de son développement. Cette humanité-là s'appauvrit. Comment peut-elle tenir à la verticale Combien d'enfants sont morts de malnutrition depuis que nous avons commencé notre entretien ? Quelle est la grande responsabilité morale de ceux qui sont riches, la minorité de nantis que constituent la dizaine de pays et dont nous sommes ! Arnold Toynbee, qui a écrit l'histoire des cycles des civilisations dans Study of History, a déjà soutenu que la caractéristique majeure de notre humanité actuelle n'est pas tant la capacité autodestructrice dont nous venons de parler que la responsabilité nouvelle de cette partie développée de l'humanité de sortir l'autre de cet état périanimal pour l'élever à un niveau de vie proprement « humaine ». Cette inquiétude va se faire de plus en plus pressante dans les prochaines années. Et passons vite, si vous le voulez bien, sur la torture et le terrorisme qui se répandent de plus en plus comme moyens d'action politique. À l'échelle planétaire, notre humanité n'est pas belle à voir..

J.B. J'ai entendu à Radio-Canada un philosophe, critique de notre milieu, reprocher à nos penseurs d'être trop obsédés par les problèmes du Québec. Parmi les noms qu'il citait se trouvait le vôtre. Il faisait allusion à vos papiers dans Le Devoir sur la crise constitutionnelle.

G.B. Je répondrai qu'il y a un aspect important de ma vie et de mon oeuvre que ce jeune homme ne connaît pas. Quotidiennement, je pense à la fin du monde. Et, de plus en plus, à sa faim. C'est un sentiment oppressant pour qui continue à se bien nourrir. Nous savons encore que la guerre atomique a failli se produire une couple de fois par une erreur mécanique de détection. Au moment des grandes 
crises - celle de Berlin en 1948, celle de Cuba en octobre 1962 qui a abouti presque à la collision des deux Supergrands - j'ai vécu intensément cette angoisse, sachant ce qui était en cause.

Lors de la crise des missiles, l'angoisse était même perceptible dans la population. Mes enfants de douze et dix ans étaient revenus de l'école en demandant : « Est-ce vrai, papa, qu'on va avoir la guerre ? » J'ai dû les rassurer sans être certain de ma force persuasive. Je suivais de jour en jour l'évolution de cette crise, collé à la radio et à la télé, dépouillant tous les journaux du monde. Le soir de la réponse de Kkroutchev à l'ultimatum de Kennedy d'avoir à s'en retourner avec sa flotte et ses missiles à défaut de quoi c'était l'affrontement militaire, j'étais commentateur de l'événement à la télé de Radio-Canada à 20h20. C'est vous dire avec quelle intensité j'ai vécu ce drame. Eh bien ! allez dire à ce confrère que, pour Gérard Bergeron, le fait humain fondamental est d'abord et avant tout la conjugaison de ces deux effroyables réalités : la menace de la guerre atomique et le sousdéveloppement éhonté de la majorité humaine de la planète.

\section{J.B. La question québécoise là-dedans ?}

G.B. Toute relative, comme vous vous en doutez bien, et même triviale en comparaison de ce que nous venons d'évoquer. Mais bien réelle, aussi. Ces deux réalités fondamentales n'interdisent pas la conscience de sa biologie élémentaire, de sa famille, de son milieu de travail, de ses amis, de son pays. Il peut ne pas y avoir coïncidence entre l'aire du plus grand intérêt et l'aire de l'intervention possible. On n'a pas à rejeter l'un [87] parce qu'on privilégie un autre à un moment donné. Rien ne peut justifier le silence ou l'abstention vis-à-vis de son propre pays. Et puis ici, on peut au moins nourrir l'illusion qu'on peut contribuer à y changer quelque chose, fût-ce si peu ! Et, si ce n'est pas nous, qui le fera ? Il y a même un devoir d'aboyer de temps en temps. Mais si on était vraiment « logique » dans ses angoisses sur le sort de l'humanité, on paierait davantage de sa personne et de ses deniers. 
J.B. L'internationaliste n'est-il pas porté à broyer du noir ? Quel est le plaisir à travailler au jour le jour dans le vif de l'actualité politique se faisant, la grande comme la petite, d'ailleurs et d'ici ?

G.B. Le «plaisir », c'est de se sentir pleinement contemporain! Pour moi, la dernière page de l'histoire s'écrit au jour le jour dans le bulletin de nouvelles ou la dernière édition du journal. Je suis hanté par le problème du demain et de l'aprèsdemain. L'an 2000 me fascine, par sa rondeur quasi magique. Dans un cours de doctorat sur la prospective, j'ai analysé, avec mes étudiants, le mythe naissant de l'an 2000. Que nous réserve-t-il, après les années optimistes de la décennie 60, les inquiétudes angoissantes des années 70, la recherche d'une sérénité et d'une plus grande sagesse peut-être des années 80 à cause des crises et des raretés qui s'annoncent, y compris la rareté spirituelle. Je vis donc dans une espèce de familiarité avec l'an 2000. J'ose espérer le voir, être présent à ce grand rendez-vous de l'humanité, en espérant encore plus qu'elle soit plus belle que celle d'aujourd'hui. Sinon, pourquoi nos enfants continueraient-ils à faire des enfants ? 
[89]

GÉRARD BERGERON.

de l'autre côté de l'action.

$4^{\mathrm{e}}$ entretien

LE JOURNALISTE

«... de l'autre côté de l’action »

Retour à la table des matières 
J.B. Nous allons donner le titre "Le journaliste" au présent entretien, bien que je sache que vous ne vous considérez pas comme un véritable journaliste.

G.B. Le journalisme n'est pas mon métier, ni mon activité principale. Je suis un universitaire, c'est-à-dire un enseignant et un chercheur, qui écrit et dont une partie de ce qu'il écrit paraît en première publication dans les journaux. Je suis quelqu'un qui envoie des lettres aux journaux, principalement au journal Le Devoir. Mes lettres, un peu plus longues que celles des tribunes libres de lecteurs, y pont aussi plus fréquentes, du moins par moments. Elles constituent des espèces de chroniques libres, car si, par périodes, je suis assez ponctuel, il en est d'autres encore plus longues pendant lesquelles je m'enferme dans des travaux qui n'ont rien de journalistique. Que ces chroniques ou analyses, publiées d'abord en la forme d'articles de journaux, servent ensuite à confectionner des livres, qui tombent sous la catégorie générale de recueils ou d'essais, cela montre pourquoi elles ne relèvent qu'indirectement du véritable journalisme. C'est après ces précisions que je vais répondre à vos questions sur « le journaliste. »

J.B. Je pourrais vous demander : Comment en vient-on à faire ce journalisme-là ? Pour vous, c'est tout de même la reprise d'une activité pratiquée pendant vos [92] études universitaires et que vous n'avez jamais mise au rancart. Dans quelles circonstances y êtes-vous revenu ?

G. B. Je le constate avec le recul : ma vie a été ponctuée de rencontres décisives. La rencontre avec le Père Lévesque m'a orienté du côté de la carrière universitaire ; celle avec André Laurendeau déterminera mon retour au journalisme ; plus tard, Gérald Godin me convaincra que mes articles vaudraient d'être republiés en volume. Nous sommes en juillet 1956. Radio-Canada m'invite à une émission d'affaires publiques. Le sujet débattu : la reconnaissance de la Chine communiste. Parmi les autres invités, il y avait André Laurendeau. Il avait été une 
idole de jeunesse. À la fin de la guerre, presque tous les étudiants étaient partisans du Bloc populaire, dont il était le chef provincial, comme ils sont péquistes aujourd'hui. De plus, j'admirais autant l'homme que l'éditorialiste. Comme il me reconduisait à l'hôtel après l'émission, la conversation tomba naturellement sur les élections du 20 juin qui venaient de reporter au pouvoir un Duplessis plus fort que jamais. La conversation dura une bonne heure dans son auto...

À un moment donné, il me lance : «Pourquoi ne mettez-vous pas par écrit, pour Le Devoir, tout ce que vous êtes en train de me dire ? » Je lui réponds que je suis un jeune professeur, que je suis encore dans la période de rodage de mes cours de base, que je prends goût à la recherche universitaire, et qu'enfin j'avais réussi à « tuer » l'attrait du journalisme. Mais je finis par lui avouer que la tentation était vraiment forte dans ces conditions. "Succombez-y, insistait-il. Il serait bon que Le Devoir ait d'autres points de vue que ceux de ses éditorialistes ou de ses lecteurs en tribune libre ». C'était bien mon avis. Je pensais au Monde qui accueillait depuis ses débuts des collaborations de type universitaire, dont la plus assidue à l'époque était celle de Maurice Duverger et au Figaro, bien sûr, que Raymond Aron avait préféré au Monde, ayant estimé, après la courte expérience de Combat sous la direction de Camus, qu'il lui serait plus facile de s'entendre avec Pierre Brisson qu'avec Hubert [93] Beuve-Méry. De retour chez moi, je résiste une semaine. Puis, en pleine canicule, je commence à écrire sur la situation créée par la dernière victoire dupplessiste. J'accouche, au bout du compte, d'un petit essai, que je découpe en quatre articles bien tassés, et je les fais aussitôt parvenir à Laurendeau.

\section{J.B. Sous le pseudonyme d'Isocrate, pourquoi ?}

G.B. Demi par discrétion, demi par mystification ; et j'avoue que la seconde moitié était plus forte que la première! Il faut se replacer dans le contexte. La faculté où j'enseigne n'est pas très bien vue par le régime en place. Déjà le Père Lévesque et quelques professeurs, principalement Maurice Lamontagne, étaient en mauvais terme avec le parti au pouvoir. L'Université dépendait financièrement du gouvernement provincial pour d'importants appoints. Ça n'aurait pas servi la Faculté ni la cause de mes articles qu'un autre membre de la «Gang » du Père 
Lévesque manifeste son opposition. Encore que je n'attaquais pas tant le parti au pouvoir que la classe intellectuelle active dans l'action sociale et économique qui, se refusant à l'action politique, n'atteignait pas directement le régime sur le plan politique. Mais surtout, je ne m'en suis jamais caché, j'ai eu le goût d'une petite mystification. Je me suis dit que ce serait plutôt drôle, dans une société où tout le monde prétend se connaître, en particulier chez les membres de la caste intellectuelle, de voir combien de temps pourrait durer la chasse à la signature inconnue.

\section{J.B. Mais le choix d'Isocrate?}

G B. Bêtement parce que c'est le premier nom qui me vint alors à l'esprit ! Par quelle association me suis-je rappelé cet écrivain de deuxième catégorie, que nous traduisions au collège, qui était adversaire malheureux de Demosthène en réclamant l'union de tous les Grecs, y compris des Macédoniens, contre les Perses ? [94] Je l'ignore. J'apprendrai plus tard qu'il se suicida à l'âge de... 98 ans, de désespoir à cause de l'échec de sa politique!

\section{J.B. Laurendeau n'ignore pas, lui, qui se cache derrière ce pseudonyme.}

G. B. Non, et je lui demande de n'en rien dire à personne, ni au directeur du Devoir, Gérard Filion, ni à Paul Sauriol, le secrétaire de la rédaction qui mettait les articles en page. En fait, personne n'en a rien su aussi longtemps que je l'ai voulu. Cela a tout de suite piqué la curiosité : pour la première fois, peut-être, les lecteurs du Devoir se voyaient offrir des textes moins polémiques qu'analytiques. Après quelques semaines, j'ai fait paraître deux autres articles, puis un groupe de six, et ainsi de suite avec répétition pendant deux ans. J'ai vécu des moments assez cocasses. Dans les divers milieux que je fréquentais, il pouvait se trouver des gens qui, commentant les textes d'Isocrate, se demandaient qui pouvait-il bien être. Et pourquoi ce mystère ? Je feignais de chercher avec eux. Beaucoup de noms défilaient, sauf le mien! Tous s'entendaient pour dire que ça devait être un universitaire à cause de son mode d'expression. On cherchait du côté des HEC ou des facultés de sciences sociales ou de philosophie. Certains pensaient même que 
c'était le pseudonyme d'André Laurendeau. Ne signait-il pas déjà des billets du pseudonyme de Candide ? Pourquoi pas un deuxième ?

À un moment donné, nous sommes une dizaine d'amis attablés chez Kerhulu dans le Vieux-Québec. L'inévitable sujet de la politique revient sur le tapis. Quelqu'un évoque de récents papiers d'Isocrate et, comme il est encore question de son identité, un autre lance mon nom à la blague. Tout le monde part à rire ! Pour tous, j'étais le gars en orbite dans la politique internationale et qui venait justement de faire des boutades sur le caractère provincialiste des intellectuels d'ici. C'est ainsi qu'homme d'un milieu qui ressemblait à une grande paroisse, j'ai pu écrire copieusement sur notre politique de cet été et automne [95] 1956, l'analyser, le décortiquer en toute quiétude et en complet anonymat. Une petite blague assez inoffensive comme vous voyez.

\section{J.B. Mais le pot aux roses a fini par être découvert.}

G.B. Par René Tremblay, maintenant décédé, un universitaire de ma promotion, économiste, futur député de Matane au fédéral et ministre sous Pearson. Nous nous rendions en voiture à Montréal. Nous causions de politique... Comme mes opinions étaient - assez naturellement - près de celles d'Isocrate, que je semblais même les avoir retenues assez fidèlement, et que je les prolongeais puisque j'étais en train d'ébaucher une nouvelle série sans trop m'en rendre compte, René se tourna tout à coup vers moi (nous venions de passer Louiseville) : " Isocrate, c'est toi ! » Je ne pouvais y contredire. J'ai alors trouvé loyal que le directeur du Devoir, Gérard Filion, en soit informé en lui disant que la mèche avait été éventée. «Qui êtes-vous dans la vie ? », me demanda-t-il. Il ne le savait pas du tout. Il ajouta : «Vous pourrez dire que Laurendeau est un homme de parole. Il n'a jamais cédé aux pressions de dévoiler votre nom ». Il semblait que des gens de l'Union nationale voulaient aussi le savoir. Dans l'organe du parti libéral, La Réforme, Jean-Louis Gagnon se demandait en éditorial «Qui est Isocrate ? » J'ai continué à signer de ce pseudo(et même en forme raccourcie, « Iso », pour quelques courts billets) même après que mon nom ait été connu des cercles d'initiés. 
On traversait alors une période d'effervescence : la défaite de Saint-Laurent et l'arrivée en force de Diefenbaker, la rénovation du parti libéral provincial, l'élection de Jean Lesage à la tête des troupes libérales etc. En 1957, l'historien Robert Rumilly écrit un livre de propagande, L'infiltration gauchiste au Canada, où il vitupérait, nommément, tous les opposants à Duplessis. D'Isocrate, il écrivait qu'« il n'est nul autre que Gérard Bergeron, professeur de science politique à l'Université Laval ». Comme s'il avait lui-même découvert le secret !

J.B. À relire la première fournée d'articles d'Isocrate, votre côté internationaliste transparaît : par la distance que vous prenez de votre milieu, même quand vous en traitez.

G.B. Quand on en fait la remarque ce n'est pas d'habitude dans le sens d'un compliment. C'est peut-être dans ce sens-là que le théoricien l'emporte sur le citoyen, voyant plus grand et relativisant. Beaucoup d'intellectuels, même formés aux sciences sociales, ont tendance à tout ramener à leur situation immédiate et environnementale. C'est ce côté qui m'a déjà fait définir comme un professeur qui enseigne au Devoir et à l'Université Laval...

\section{J.B. Partez-nous du Rassemblement.}

G.B. C'était à la même époque des années 56, 57, 58. J'étais d'un groupe de jeunes intellectuels sociaux qui s'étaient rassemblés sous l'étiquette la moins compromettante qui soit : Le Rassemblement. Des gens qui allaient devenir importants en faisaient partie : Pierre Elliott Trudeau, entre autres. Pierre Dansereau allait en devenir le premier président. Il vient d'évoquer, dans des entretiens semblables aux nôtres, ses souvenirs de l'époque du Rassemblement ${ }^{7}$. Après trois ans, le

7 Pierre Dansereau, L'écologiste aux pieds nus, Montréal, Nouvelle Optique, 1981, p. 71-5. Voir Du duplessisme au johnsonisme (Parti pris, Montréal, 1967), les pp. 120-135 ; ou la deuxième édition, augmentée, sous le titre $D u$ duplessisme à Trudeau et Bourassa (même éditeur, 1971), les pp. 128-143. 
mouvement était à bout de souffle et d'inspiration. J'en fis alors l'analyse, presque une psychanalyse dans Le Devoir. J'y disais crûment cette incapacité d'une génération à agir politiquement. Des membres, et non des moindres, ont réagi : «Comment se fait-il que Bergeron, avec nous depuis le début, traite de la question comme s'il n'était pas lui-même en cause ? " Ce point de vue d'analyste de l'extérieur n'était pas prisé. Il m'aurait fallu paraître plus « pogné » dans ma psychologie. La réalité était que je n'avais pas la moindre velléité de m'engager dans l'action politique directe. Je ne parlais tout de même pas de ces questions comme s'il se fut agi du Vénézuela ou de la Bulgarie !

[97]

\section{J.B. Il a bien fallu que vous ressentiez une certaine urgence pour plonger dans le journalisme en 1956 ?}

G.B. L'aurais-je fait sans la rencontre opportune avec Laurendeau ? Pas sûr. Quoi qu'il en soit, je me disais : «Il faut faire dès cet été 1956, avant qu'on passe et qu'on pense à autre chose, l'analyse critique du scrutin du 20 juin », qui avait littéralement assommé beaucoup de monde dans la province. Le réflexe critique tardait. J'ai clairement eu l'impression d'exercer une suppléance. Ces élections allaient aussi, dans les semaines qui ont suivi mes articles, susciter l'intervention publique de deux abbés, Gérard Dion et Louis O'Neill. Dans une revue destinée aux prêtres engagés dans l'action sociale, ils publièrent un texte dru, pamphlétaire, exprimant leur indignation sur la façon immorale dont les dernières élections s'étaient déroulées et sur l'absence de réaction des autorités religieuses. Cette longue lettre sera publiée dans Le Devoir et elle aura un impact extraordinaire ; plus tard Isocrate fera le bilan de cet impact ${ }^{8}$. Le fait d'attaquer publiquement le silence des clercs les plus en autorité devenait tout un événement dans une société encore à façade cléricale, à fortiori si les auteurs de la contestation appartenaient eux-mêmes au clergé. Inutile de vous dire que cette lettre allait éclipser les pre-

8 Gérard Dion et Louis O'Neill, Le chrétien et les élections, Montréal, Éditions du Jour, 1957. Voir les deux livres de Bergeron signalés à la note précédente : le premier, aux pp. 111-120, le second, aux pp. 119-128. 
mières interventions d'Isocrate qui relevaient moins de l'indignation morale que de l'urgence politique.

J.B. Une phrase m'avait frappé à la lecture du livre qui réunit tous ces articles, Du Duplessisme au johnsonisme : «On ne demande pas à un homme politique d'être moral mais d'être efficace. "

G.B. Ce qui ne devait pas s'entendre comme un éloge de l'immoralité ! J'ajoutais en effet que l'efficacité, pour être réelle, devait se fonder sur des valeurs sociales.

J.B. En invitant à l'action politique, pensiez-vous à des gens précis, ou alliez-vous à la pêche, comme on dit ?

G.B. Je pensais à des gens précis manifestement taillés pour l'action politique et dont plusieurs étaient dans ce que j'appelais « la salle d'attente » du Rassemblement. Des gens qui étaient, le reste de leur carrière allait le démontrer, en disponibilité d'action politique. Je n'adressais pas mes articles à des personnes prises individuellement, mais à des catégories d'intellectuels qui étaient alors en pleine frustration. « Un véritable manteau de plomb était tombé sur le Québec », comme je l'écrivis alors en cette seizième année du régime duplessiste, interrompu par le gouvernement Godbout pendant la guerre. Le message était simple : Il faut faire sauter le régime Duplessis de son vivant (et du nôtre !), ne pas attendre ce qui est arrivé finalement, « la crise cardiaque la plus importante de toute l'histoire de la Confédération » disait je ne sais plus qui ; vous êtes taillés pour l'action politique et frustrés de ne pas trouver à employer cette énergie ; emparez-vous du parti libéral de $\mathrm{M}$. Lapalme, faites-en quelque chose comme vous y invite déjà une certaine gauche de ce parti : Jean-Marie Nadeau, Jean-Louis Gagnon, Paul Gérin-Lajoie, et d'autres moins connus. 


\section{J.B. Comment expliquer que votre génération se soit longtemps arrêtée} au seuil de la politique?

G. B. Je dirais qu'ils s'y préparaient tout en se refrénant d'y entrer. Surtout, ils nourrissaient le rêve stérilisant et impossible d'un parti qui leur convienne, tout en ayant conscience qu'ils ne pourraient pas l'établir. Ce modèle de parti existait bien, mais à l'extérieur du Québec : la CCF. Je leur reprochais justement leur incapacité à infiltrer le parti libéral à défaut de pouvoir acclimater ici la CCF ou d'y former un parti correspondant. D'autant plus que le parti libéral était en vacance de leadership et s'apprêtait à un grand renouvellement grâce à sa Fédération établie en 1955. C'était le temps d'agir, véritablement. La structure d'accueil existait.

\section{J.B. Votre appel a quand même dû susciter des réactions de la part de} ceux à qui il était adressé ?

G.B. Plutôt faibles, en forme de réticences plutôt. Parmi les plus réticents étaient P. E. Trudeau, qui inclinait vers la CCF sans s'y engager, comme dans un rêve, et René Lévesque, ce lone wolf, ce franc-tireur qui allait trouver son espèce de Chemin de Damas lors de la grève des réalisateurs de Radio-Canada, en 1959. Il aurait dit assez crûment, dans les couloirs de Radio-Canada, que c'était un peu fou de croire qu'on pouvait faire quelque chose avec un vieux parti comme les libéraux. Mais il allait adhérer à l'équipe Lesage en janvier 1960. Vous savez le reste. Quant à Laurendeau, il se maintenait dans le camp des sceptiques. C'était compréhensible : il s'était colleté, lui, à l'époque du Bloc populaire, avec ce dinosaure à deux têtes qu'était le parti libéral du Canada et du Québec. Son engagement civique à venir allait être d'un autre ordre, à la tête de la Commission BB, en co-présidence avec Davidson Dunton : une triste mort, dramatiquement prématurée et dont les conséquences politiques ne peuvent être qu'imaginées.

J.B. J'ai relu ces textes des années 1950 . Votre critique était dure, très dure. Vous la destiniez pourtant à des amis quasiment. 
G.B. C'est une constante que je crois n'avoir pas démentie. Je fais des critiques d'autant plus dures qu'elles sont à l'endroit de gens ou de formations avec lesquels je me sens plutôt en communauté de valeurs politiques. Cette critique reste très acceptable pour eux parce qu'elle ne vient pas d'un adversaire en mal de les remplacer ou de les écarter. Dès le début, j'adoptais cette position d'être de l'autre côté de l'action politique, dans ces espèces de zones indéfinies de la pré-action en vue de l'action ou du jugement après coup de l'action des autres. Mes critiques se situent au plan de leur programme et de leurs objectifs déclarés, du moins dans ce que j'estime valable de ce qu'ils nous proposent. Bien sûr que ça peut déconcerter des adhérents ou des partisans, mais ceux avec lesquels je discute ne doutent pas de mon désintéressement, à ce qu'il m'en revient tout au moins. À l'époque de mes [100] premiers articles, le connaissais assez bien ce milieu intellectuel qui était plus restreint qu'aujourd'hui. Je voyais d'autant plus clairement les blocages, l'incapacité d'agir alors que plusieurs étaient visiblement attirés par la carrière politique. On peut dire que j'ai bien connu plusieurs éléments forts et des têtes d'affiches des futurs gouvernements de la Révolution tranquille, du French Power et du gouvernement du PQ depuis 1976.

\section{J.B. Pourquoi le mode d'intervention journalistique et non d'autres tri- bunes?}

G.B. Sans doute parce que j'aime écrire et que le journalisme est plus naturel à un individualiste de mon espèce. L'avantage de l'article de journal, outre d'exiger peu de temps de son auteur, c'est de lui permettre d'atteindre un plus large public que celui des plus grands amphithéâtres ou des plus gros tirages de livres. Quand j'écris un article, je n'engage personne d'autre que moi et je ne consulte que moimême. Et puis je nourrissais ce goût de l'écriture depuis toujours, mis de côté mais avec l'arrière-pensée que la carrière universitaire le permettrait à point nommé. Le point nommé est survenu par la conversation avec Laurendeau en 1956.

\section{J.B. Pourquoi écrit-on finalement?}


G.B. Je ne vous sortirai pas la salade de l'engagement et du service public. Je le fais d'abord parce que j'aime ça ! Pour mettre de l'ordre dans mes pensées aussi. On ne comprend jamais mieux une chose que lorsqu'on la met par écrit. Ces hommes de la parole que sont les politiciens manquent assez souvent de cohérence, pour ne pas parler de leurs contradictions plus visibles. Leur pensée se fait, se défait et se refait constamment, au gré des publics et de l'improvisation, et surtout sous la pression des problèmes se renouvelant constamment. Pour les hommes d'écriture, y compris de [101] l'écriture fréquente du journalisme, c'est un moyen plus assuré, mais aussi plus compromettant. Par l'écrit, le manque de clarté ou de cohérence est plus frappant. Il suffit de se souvenir de ce qu'on a écrit la veille ou l'avant-veille... Pensez aux articles de Trudeau dans Cité Libre, que des adversaires exhument de temps à autre. Souvenez-vous de la résurrection des éditoriaux de Ryan à laquelle s'est adonné le PQ lors des premiers mois de vie politique du chef du PLQ.

\section{J.B. Le public du Devoir n'est pourtant pas le plus nombreux Pourquoi avoir choisi ce journal ?}

G.B. Parce qu'il est unique. Le Devoir n'est pas un grand journal, mais c'est aussi plus qu'un journal, selon une formule d'apparence paradoxale qui résume bien ma pensée. Quand on en parle à des Canadiens anglais qui le connaissent un peu ou à des étrangers qui ne le connaissent pas du tout, ils croient qu'il s'agit d'un journal de classe internationale comme le New-York Times, l'ex-Herald Tribune, le Washington Post, le Guardian, le London Times, ou le plus connu chez nous, Le Monde, etc. Le Devoir n'est pas de cette classe. Un grand journal a de grandes ressources, se met à l'écoute du monde, possède des reporters aux points chauds de la planète. Un grand journal est une entreprise très coûteuse qui a une clientèle fixe et qui produit des éléments ou pièces d'archives d'une histoire en train de se faire. C'est un instrument de travail : non pas seulement une référence, mais de recherche préliminaire sur toutes espèces de sujets.

Le Devoir n'a ni cette taille, ni ces moyens, ni cette utilité. Mais il est probablement le journal le plus important au Canada même si le Globe and Mail, avec 
lequel on le compare habituellement, semble plus près de la notion de quotidien de classe : gros moyens, vaste public, prétention de couverture pancanadienne, distribution régionale. Malgré son point fort, sa section économique, le Globe m'apparaît jouir d'une réputation surfaite et reste assez loin de la classe internationale, à mon avis, entre autres raisons à cause de sa faiblesse éditoriale. Le Devoir n'est pas un grand [102] journal, mais il est plus qu'un journal, ai-je dit. C'est une institution unique, parce qu'il est à l'image générale, mais non à la ressemblance stricte de notre société. Le Devoir inverse l'ordre des priorités : en dépit de l'effort considérable tenté naguère par Jean-Marc Léger, il reste déficient et sous-équipé sur le plan de la nouvelle internationale, s'en remettant comme les journaux moyens aux agences de presse ; il n'est pas en ce domaine un journal de records, d'archives, de future documentation pour la recherche. Encore que sa nouvelle parlementaire d'Ottawa et de Québec, même par un petit nombre de journalistes, a toujours été d'une qualité exceptionnelle, d'aussi loin que je me souvienne. Il a même forcé à se réveiller $L a$ Presse et Le Soleil, qui sont encore loin de l'égaler avec des moyens supérieurs.

\section{J.B. Par quoi est-il important?}

G.B. Par son éditorial et par sa page éditoriale toute entière, ce qui ressort davantage d'un hebdo culturel ou d'une revue spécialisée. Le Monde, par exemple, n'a pas d'éditorial régulier, simplement une petite nouvelle commentée sur le plan international, son célèbre «Bulletin de l'étranger ». Les colonnes éditoriales du New-York Times sont courtes, c'est par des chroniques sectorielles et signées que se fait principalement l'analyse. Le Devoir, c'est l'éditorial avant tout, l'expression de la pensée nationaliste canadienne-française tant sous Henri Bourassa que sous Georges Pelletier et Gérard Filion. Puis il y eut le directorat de Claude Ryan à partir de 1964 : pour la première fois de son histoire, Le Devoir s'est retrouvé, dans sa pensée éditoriale, en réticence, voire en opposition aux forces nationalistes du milieu.

\section{J.B. Que voulez-vous dire par « plus qu'un journal »?}


G.B. Cela que j'ai commencé à dire, plus un lieu de rencontre, presqu'une chapelle. Il jouit d'une fidélité quasi religieuse : [103] et les plus fidèles sont peut-être ses détracteurs qui marquent leur mauvaise humeur en se désabonnant et en le jetant au panier mais y reviennent toujours! Tout se passe comme si le Québec français avait besoin de cette mise au point quotidienne. Le Devoir remplit cette fonction également par un effort particulier pour suivre la vie littéraire, artistique, culturelle de notre milieu. Mais sur ce point, l' «effort »manque de continuité dans les moyens et, à vrai dire, d'idées directrices, ce qui se voit dans les résultats qui pourraient être bien meilleurs.

J.B. Je pense que la formule du "texte-dossier" de la page 5 - quand l'éditorial était à la page 4 - Des idées, des événements, des hommes, est une autre caractéristique capitale du Devoir.

G.B. Et comment ! Cette section est un sacrifice extraordinaire de la part d'un journal à moyens limités : une page entière consacrée aux collaborateurs libres de l'extérieur, avec des articles de toute espèce, des analyses plus ou moins longues et qui sont souvent de véritables dossiers, quelquefois en deux ou trois suites, écrits par des hommes d'action de toute espèce, des spécialistes ou des experts, ou ce qu'on pourrait appeler des consciences individuelles. À l'époque de Filion, dans les années 1950, Laurendeau pensait à ce type de collaboration; c'est pour cela qu'il a suscité et si bien accueilli Isocrate. Et depuis lors, ce mode de collaboration s'est généralisé et même s'est institutionnalisé dans la page en question.

\section{J.B. Isocrate a été le Premier du genre. Mais Gérard Bergeron a aussi été le propagandiste de la formule, si je peux m'exprimer ainsi.}

G.B. Ça s'est passé en public, en novembre 1960, alors que j'avais cessé d'écrire deux ans et demi plus tôt. C'était à l'occasion d'un dîner à Québec, auquel Gérard Filion et son équipe avaient convié plusieurs, [104] invités dont Jean Lesage, à célébrer le 50e anniversaire du Devoir. C'était au début de notre résurrec- 
tion collective tant attendue. L'ambiance nouvelle se sentait. On m'avait demandé de dire ce que je pensais du Devoir et de sa vocation. Je parlais d'après des notes. Je soutenais qu'il était important que d'autres voix se fassent entendre dans les pages du journal et qu'elles soient aussi celles d'associations, de groupes, d'organismes divers. Pourquoi ne se serviraient-elles pas de la tribune-carre-four du Devoir ? J'ajoutais que l'occasion était très belle, que le nouveau gouvernement était très accueillant aux idées neuves, qu'il les sollicitait même. Il fallait lui faire connaître nos doléances. Le Devoir était le véhicule tout désigné pour cela. Je me souviens de ma dernière phrase : «Le Devoir se trouve devant cette chance inouïe de devenir une officine de pré-législation sans cesser d'être une tribune d'opposition ${ }^{9}$. »

\section{J.B. Vous êtes un peu de la maison ?}

G.B. Pas au sens intimiste de votre question. Je n'y ai jamais eu, encore moins réclamé de statut professionnel ou privilégié. Je fais comme tout le monde : j'écris un texte, je le mets à la poste, la direction en fait ce qu'elle veut. Je joue le jeu. Parfois des gens me demandent de dire un bon mot pour eux pour accompagner leur texte. Je leur réponds : « Pourquoi le ferais-je pour vous, je ne le fais pas pour moi ? Comme les miens, votre texte prendra le chemin de la corbeille d'attente. » J'agis à titre de lecteur citoyen.

\section{J.B. Vous avez côtoyé plusieurs équipes de rédaction.}

G.B. Connu plus que côtoyé et sans avoir d'autre lien que ce que je viens de vous en dire. On peut dire que depuis Isocrate, certains m'associent à tort au journal. Suffisamment, en tout cas, pour que les lecteurs ne s'étonnent pas que l'intervienne publiquement dans des moments difficiles, par attachement à l'institution. [105] Je l'ai fait en deux occasions récentes : lors du vide créé par le départ de

9 Incertitudes d'un certain pays, Québec, Les Presses de l'Université Laval, 1979, chapitre II. 
Ryan en 1978 et lors de la grève navrante du printemps 1981, alors qu'il m'est apparu que ceux qui voulaient sincèrement sauver et renforcer le journal étaient peut-être en train de le tuer.

\section{J.B. Avez-vous vraiment craint pour sa survie?}

G.B. Oui, si la grève se prolongeait indûment et amenait la fermeture pendant les mois d'été. Et je ne suis pas sûr, au moment où nous nous parlons, en ce mois d'août 1981, qu'il va prendre le virage de la rentabilité à l'automne. Ça va se jouer bientôt. Vous êtes d'une génération qui n'avez pas connu les sauvetages périodiques de ce journal qu'il a fallu effectuer autrefois, par des souscriptions publiques et des campagnes de financement. Le Devoir mendiait littéralement dans les années 1950. Peut-être risquons-nous de revenir à une situation semblable ? Le milieu s'appauvrirait irrémédiablement si on le perdait. La grande presse reconnaît ce rôle unique du Devoir. La Presse et Le Soleil s'étaient empressés de publier mon article du temps de sa malencontreuse grève.

\section{J.B. Personnellement vous perdriez votre instrument d'intervention pu- blique. Je vous imagine mal dans les pages d'un autre journal.}

G.B. Le Devoir constitue un espace-jonction entre divers courants et opinions qui agitent notre milieu : dans les mouvements sociaux, les milieux d'affaires, les maisons d'enseignement, les associations diverses. C'est le relais mitoyen de la circulation des idées au Québec, dans un contexte de très grande liberté. La fameuse page-dossier accueille, une fois sur deux, des voix distinctes et même opposées à la position officielle du journal. C'est un carrefour d'opinions, un choc perpétuel d'idées, obéissant à la règle de la confrontation sur la place publique comme se l'est imposé Radio-Canada en vertu de son statut pour les émissions [106] d'affaires publiques. Mais sans tomber dans le maniérisme de Radio-Canada qui, dans son obsession de faire s'équivaloir numériquement le pour et le contre, présente souvent des figurants fictifs ou n'ayant pas grand chose à dire. Le Devoir, y compris celui de Ryan, a été très hospitalier à toutes ces voix disparates. Il ma- 
nifeste un grand libéralisme de pensée propre aux institutions culturelles de ce bas monde. Tout cela, évidemment, à l'intérieur de certaines limitations matérielles (espace, fréquence, niveaux du discours proposé, etc.).

\section{J. B. N'avez-vous pas, il y a quelques années, eu une collaboration pério-} dique?

G. B. Me faisant plutôt abondant après le 15 novembre 1976, je m'en suis excusé auprès de la direction. Le directeur d'alors, Claude Ryan, avec son rédacteuren-chef, Michel Roy, prirent la décision inverse : ils m'invitèrent à une collaboration régulière au rez-de-chaussée de l'éditorial du samedi matin. Pendant trois mois, j'allais y exposer toutes sortes de points de vue pas toujours conformes à la pensée éditoriale à quelques centimètres plus haut. Peu de journaux auraient accepté un point de vue divergent ou nuancé du leur à quelques lignes de l'éditorial. C'est plutôt moi qui m'en suis trouvé gêné. Nous traversions des moments très chauds : la Loi 101, la querelle des comptes économiques entre les gouvernements de Québec et d'Ottawa. Et il me semblait, ainsi qu'à d'autres, que l'éditorialiste Ryan défendait des positions d'une objectivité de plus en plus douteuse. Il préparait manifestement son entrée en politique. Cette promiscuité dans les circonstances pouvait devenir gênante. J'ai profité de l'arrivée de l'été pour me retirer et je n'ai pas repris cette collaboration régulière à l'automne. D'autant plus que ça devenait très contraignant pour moi, cette périodicité hebdomadaire.

\section{J.B. Considérez-vous avoir fait du bon journalisme pendant ces trois mois d'articles hebdomadaires?}

106

G.B. Ce n'était pas véritablement du journalisme, donc pas du bon journalisme. Le bon journaliste doit être à la pointe des événements. Sa mécanique analytique doit entrer en jeu au plus chaud de l'actualité qui va vite. Le champ à balayer est celui des vingt-quatre, quarante-huit, soixante-douze dernières heures. La réceptivité est alors très grande chez le lecteur. Cette écriture « à chaud » permet au 
journaliste d'ajuster constamment sa pensée au rythme des événements. Arrivant en retard, le chroniqueur d'occasion est forcé de se situer à un niveau de généralité et de globalité pour tenter de saisir des dynamiques évolutives, selon un rapport moins direct à l'actualité immédiate.

J.B. Cela se reflète sur la langue de vos articles qui s'apparenterait à celle de l'essai plutôt qu'au caractère direct de l'article de journal.

G.B. Oui, sans doute. J'ai toujours admiré le journaliste qui écrit dans le stress éprouvant de l'heure de la tombée, bousculé par le chef de pupitre ou le directeur de l'information et disposant d'un espace limité. L'éditorialiste est sujet à une contrainte moindre mais du même ordre. Il doit écrire vite et court et que « le meilleur passe »! J'éprouve infiniment de respect pour ce métier-là. Le chroniqueur libre n'écrit que de son propre mouvement, lorsqu'il est en humeur et en disponibilité pour le faire. Il serait donc normal, y mettant plus de soin et de temps, que le produit final soit un peu plus brossé. Olivar Asselin, qui passait pour la sommité journalistique de son époque, disait : «Dans les journaux, on écrit à la machine : ça se voit. »

Je ne peux pas parler de l'actualité découpée en tranches quotidiennes puisque je ne suis pas dans la bousculade de cette actualité. Je dois plutôt traiter de la dynamique générale des événements, ce qui exige déjà plus d'attention de la part du lecteur. Cette distanciation m'amène aussi à faire des projections sur ce qui s'en vient. Cet élargissement des points de vue relève plutôt du genre de l'essai. Des gens me disent qu'ils découpent mes articles et les lisent en soirée ou la fin de semaine. Ce type de papier exige [108] beaucoup d'un lecteur de journal, même d'un lecteur du Devoir.

J.B. Vous portez une attention particulière à la langue. Cela se voit. Admettriez-vous une prétention littéraire?

G.B. Je l'avouerais cette «prétention » comme vous dites. J'aimerais écrire une langue qui serait littéraire, sans en avoir l'air, qui marierait l'attraction de 
l'image à la précision du concept. Je m'applique à alléger mon style. Les travaux de la spécialisation désapprennent à écrire simplement ! La pratique d'une langue scientifique et conceptualisante, d'intention rigoureuse finit par entraver l'expression écrite normale. L'idéal serait l'emploi d'une bonne langue, aisée, mais sans recourir aux facilités d'un simplisme verbal qui m'est toujours apparu démagogique.

J.B. Ces types de collaboration d'universitaires à des journaux, si vous l'avez introduite ici, vous n'en avez pas inventé le genre. En Europe, en France, en particulier, c'est d'une pratique assez fréquente, comme le cas d'André Siegfried dans votre jeunesse.

G.B. André Siegfried a été l'un des exemples les plus notables. Professeur à l'École des Sciences Po. puis au Collège de France, il était chroniqueur attitré au Figaro dès avant la seconde Guerre. J'ajoute que ses livres ont contribué à confirmer ma vocation naissante de sociologue politique : spécialement son $\mathrm{Ca}$ nada, puissance internationale, mais aussi ses livres classiques sur les États-Unis, la Grande-Bretagne, la Suisse, etc. Au sortir de la Guerre, il y eut toute cette presse d'idées autour de journaux comme Libération, Combat. Des noms très prestigieux de la littérature écrivaient, comme Sartre et Camus, à côté d'intellectuels comme Raymond Aron qui continue encore aujourd'hui à L'Express, dont il est président du comité éditorial, de Maurice Duverger et d'Alfred Grosser au Monde, d'économistes comme André Philip et Alfred Sauvy... [109] Nous n'allons pas tous les nommer ! Voilà autant d'universitaires chevronnés qui pratiquaient le journalisme d'occasion. Des répondants prestigieux de l'extérieur, en somme. Pourquoi, mutatis mutandis, cela ne se ferait-il pas ici ? C'était l'avis de la direction du Devoir, de Laurendeau en particulier

J.B. Le Devoir n'est quand même pas de la catégorie des grands journaux français que vous venez de nommer? 
G.B. Pour ce qui est de l'importance relative, il n'a rien à leur envier. C'est un tremplin unique pour rejoindre la presque totalité des gens influents de ce milieu. Dans presque tous les centres de décision se trouve quelqu'un qui lit Le Devoir. Vous allez m'accuser d'entretenir une mythologie ! Elle exigerait une longue analyse, d'autant plus qu'elle comporte un envers de médaille, comme toutes les mythologies en opération où l'on trouve des réputations surfaites. Cela vaut autant pour les institutions que pour les individus. Pour clore ici, je ne peux que revenir à la proposition de départ : pas un grand journal mais plus qu'un journal. Un instrument irremplaçable. Ce serait un véritable deuil national de le perdre. Ce serait terrible de perdre cet espace unique pour la circulation des idées. Mais le pire serait de ne pas pouvoir vraiment le remplacer.

J.B. Depuis plusieurs années, vous rassemblez en volumes les articles publiés dans Le Devoir. Ces livres, d'ailleurs, trouvent preneurs. Peut-être un indice du fait que ce sont de petits " essais » ? Mais cela s'est produit plus tard.

G.B. Par une rencontre fortuite avec Gérald Godin, alors recherchiste à l'émission Aujourd'hui à l'été 1966, dans un couloir de Radio-Canada. Membre de l'équipe de Parti pris, Godin avait la responsabilité des éditions du même nom. Il me lance à brûle-pourpoint : «Que diriez-vous si nous publiions les articles d'Isocrate ? » [110] Je pars à rire ! Il y avait de quoi : mes derniers papiers dataient de 1963 et il s'était écoulé cinq ans entre ceux-là et les tout premiers. Par ailleurs, là Révolution tranquille s'achevait, le groupe Parti pris ne paraissait pas tellement accordé aux vues que je défendais depuis quelques années. Godin écarte ces objections : «Au contraire, me dit-il, avec beaucoup de gens de ma génération, j'ai appris la politique dans Isocrate. En outre, nos éditions lancent une nouvelle collection en publiant les carnets politiques de Jean-Marie Nadeau, un document important pour comprendre le préambule à la Révolution tranquille. Isocrate est notre projet numéro 2. » Ces arguments m'ont convaincu. Cette possibilité ne m'était jamais venue à l'esprit. La rencontre avec l'éditeur de Parti pris, en lequel on ne pouvait voir le futur ministre du cabinet Lévesque, a eu un rôle de déclencheur comme dans le cas d'André Laurendeau. 
J.B. À l'exception des éditoriaux du Devoir - certains notables, de Filion et de Laurendeau - la littérature politique des années cinquante se résume aux articles critiques et polémiques de Trudeau dans Cité Libre et à ceux d'Isocrate dans Le Devoir.

G.B. Et, curieusement, les articles de Trudeau réunis en volume, Le Fédéralisme et la société canadienne-française ${ }^{10}$, allaient paraître en même temps que les miens : Du Dupessisme au johnsonisme ${ }^{11}$. Mon ouvrage a bien marché. "Ça se vend comme des petits pains chauds », me disait Godin. En fait, c'était le deuxième meilleur « vendeur » de Parti pris, après les monologues de Clémence, Le Monde sont drôles ${ }^{12}$, les deux premiers volumes de la maison à faire leurs frais. Clémence et moi allions vite être noyés par un autre best-seller : Nègres blancs d'Amérique ${ }^{13}$, de Pierre Vallières, qui connaîtra une fortune commerciale considérable, à l'égal presque des Insolences du Frère Untel ${ }^{14}$. Plus que deux livres, nous avons affaire là à deux phénomènes sociaux.

J.B. Situons le lecteur. Votre premier recueil de 1967 a été [111] réédité en 1971, augmenté de vos chroniques mensuelles dans Le Magazine Maclean, sous le titre rallongé Du Duplessisme à Trudeau et Bourassa. Puis, en 1977 et 1978, aux éditions Quinze, deux autres recueils autour de la prise du pouvoir par le P.Q. et de ses conséquences : L'indépendance : oui, mais... et Ce Jourlà.. le référendum. Enfin, à l'automne, vous publiez aux Presses de l'Université Laval Syndrome québécois et mal canadien, composé d'une quarantaine de textes s'articulant de près à la séquence du calendrier politique chargé des deux dernières années. N'écrivez-vous pas vos articles avec la conscience qu'ils formeront plus tard un livre?

10 Aux Éditions HMH, Montréal, 1967.

11 Aux Éditions Parti pris, Montréal, 1967.

12 Aux Éditions Parti pris, Montréal, 1967.

13 Aux Éditions Parti pris, Montréal, 1968.

14 Aux Éditions du Jour, Montréal, 1971. 
G. B. Pas dans le cas des trois premiers livres, mais c'était très net pour $\mathrm{Ce}$ Jour-là... et pour Syndrome... depuis 1978. Mes chroniques depuis lors étaient des chapitres de livres en préparation. Dans l'avant-propos de Syndrome..., j'ai cru devoir m'expliquer avec mon lecteur sur cette façon, quelque peu bizarre, de faire un livre.

\section{J. B. Cela ne rend-il pas ces écrits encore moins « journalistiques » ?}

G.B. Sans doute de façon générale bien qu'il s'agisse de textes plutôt courts. Mais certains articles ont été écrits en situation d'urgence, en quelques heures, dans le feu de l'actualité, comme tous les journalistes le font. Par exemple, l'article sur la question à poser lors du référendum s'est écrit dans la fièvre des derniers jours précédant le dévoilement de son libellé par le gouvernement. Ayant peutêtre été celui qui avait le plus disserté publiquement là-dessus, dans les années précédentes, je me suis senti obligé de repréciser ce que devrait être le libellé de cette fameuse question. J'ai écrit ce petit article d'un seul trait. J'avais fait de même quelques jours après le choc de la prise du pouvoir par le P.Q. à la minovembre 1976. Le premier ministre du Canada devait s'adresser à la population par la télévision. Il devait y exprimer le point de vue officiel de son gouvernement. [112] C'était une situation proprement historique. On pouvait craindre que si Trudeau reprenait sa notion du « Canada indivisible »comme au soir du 15 novembre, il pouvait s'ensuivre un état de tension très grand avec menace plus ou moins voilée de répression. En proie à cette inquiétude, d'urgence j'envoyai un article intitulé Un Canada indivisible, mais aussi un Québec irrépressible. L'article fut publié le matin de la déclaration du premier ministre. Il y a eu quelques autres situations d'urgence où j'ai dû agir avec la rapidité de l'éditorialiste de métier.

\section{J B. Ce sont de bons textes journalistiques, ceux-là ?}

G.B. Ça, je ne sais pas. Il faudrait vous y référer pour vous en faire une idée. Ils étaient toutefois accordés à l'événement en train de se produire. Mais il reste 
rare d'être placé en pareille situation de participation. Dans la mesure où mes textes sont lisibles longtemps après leur parution, qu'ils exigent en longueur ou en densité plus que la denrée quotidienne d'un journal, il faut opiner que ce n'est pas du « bon journalisme », comme vous dites. Pour le reste, l'appréciation relève de la critique des livres, format en lesquels ils finissent leur carrière.

\section{J.B. Comment êtes-vous perçu par les « vrais » journalistes?}

G.B. Il faudrait leur demander. Je connais peu de journalistes finalement, n'ayant pas fréquenté les salles de rédaction. Je n'ai jamais abordé ce sujet avec eux. Le milieu journalistique semble me considérer un peu comme un des leurs, comme une espèce de «membre associé »; d'ailleurs on me sollicite pour être membre de leur fédération qui compte peut-être quelques autres «journalistes » non professionnels. Ça m'apparaît d'autant plus naturel d'y contribuer que, dès les années quarante, étant encore à l'université, j'ai été un membre-fondateur de la première union de journalistes de langue française, en tant qu'amateur ou, comme on [113] dit, « pigiste » aujourd'hui. Je n'ai jamais eu d'autre statut. J'étais alors directeur du journal des étudiants de l'Université Laval, Le Carabin, et je collaborais à l'hebdomadaire Notre temps. Néanmoins, du point de vue des journalistes de métier, nous ne sommes pas de vrais journalistes. Peut-être que certains d'entre eux nous considèrent-ils comme des intrus ? Pas forcément antipathiques, mais des intrus quand même. Ou même des marginaux profiteurs. Il m'est arrivé de le constater au contact des gens du métier. Des journalistes français, à qui je parlais, un jour, de certaines vedettes universitaires collaborant à leur journal, m'ont rétorqué qu'il s'agissait d'éléments extérieurs à la profession et qu'il fallait souvent reprendre leurs textes ou les refuser...

\section{J.B. Est-ce vrai ?}

G.B. Probablement pas. Cela valait d'être cité pour ce que révèle l'intention restrictive dans la dissociation des professions. Ça me rappelle un écho parvenu d'une salle de rédaction selon lequel nous serions des gens qui « prenons le meil- 
leur du journalisme ». Ce qui, ma foi, n'est pas complètement faux. Nous ne faisons pas la carrière à partir du bas de l'échelle; nous avons acquis une certaine réputation à l'extérieur qui nous sert comme de caution ; l'heure de tombée nous est généralement inconnue, de même que la tyrannie du chef de pupitre ou du secrétaire de rédaction. C'est comme si une maison d'enseignement mettait les chargés de cours ou contractuels plus en vedette que son personnel régulier. Le journaliste de métier, même d'envergure moyenne, est plus important pour le fonctionnement du journal que n'importe quel correspondant de l'extérieur, fût-il d'une signature dite « prestigieuse ».

J.B. Votre métier d'internationaliste vous oblige à lire les grands quotidiens de l'extérieur comme Le Monde, le New York Times, le Washington Post. Trouvez-vous que la qualité des journalistes d'ici se compare à celle des journalistes américains, anglais ou français?

G.B. Redoutable question! Si je disais « oui », il me faudrait le démontrer. Si je disais «non », vous ne vous contenteriez pas d'une réponse expéditive. Je vois, dans à peu près tous les secteurs - y compris la presse sportive - des journalistes d'ici qui tiendraient leur place dans ces grandes boîtes. Je sais qu'il n'est plus de bon ton de signaler la médiocrité de nos journaux. La qualité moyenne est plus que présentable. Ce n'est plus une carrière remplie par des gens qui n'avaient pas terminé leurs études ou qu'on tenait, à tort souvent, pour des « ratés ». C'est aujourd'hui une profession qu'on choisit. La professionnalisation de la carrière, par la syndicalisation surtout, a graduellement élevé la qualité du produit en même temps que les salaires.

Il y a toutefois une trop grande rotation, malgré les avantages de la carrière. Non seulement change-t-on d'employeur, mais même de métier. Et on y revient... Et on en repart! Cette instabilité n'aide pas le rendement professionnel. Les grands journaux de l'étranger, on y entre à la suite d'une sélection serrée, on y fait ses classes avant d'avoir des affectations spéciales et des reportages de plus en plus difficiles. Et, surtout, on y reste ! Il n'est pas exclu qu'on y passe toute sa vie et qu'on accède aux charges principales de direction et de rédaction. Ici c'est plu- 
tôt l'inverse : les directions sont confiées à des gens de l'extérieur, souvent même extérieurs au métier. Je pense à Gérard Filion, Claude Ryan et Jean-Louis Roy, les trois derniers directeurs du Devoir. C'est aussi arrivé à La Presse où l'on a importé Jean-Louis Gagnon et Gérard Pelletier qui étaient de la profession, mais non de la boîte. Et, plus récemment, le cas de Roger Lemelin.

Des spécialistes de la langue pourraient porter un jugement plus sévère. Moi, je trouve que la qualité d'écriture n'est pas si mauvaise. Un journaliste écrit vite et possède toutes les excuses pour ne pas se relire trop longtemps. Mais on peut déplorer un certain manque dans la qualité technique, allant des coquilles aux mastics vraiment trop nombreux, l'abus de grandes manchettes, un manque de fini général. Mon jugement est, somme toute, modérément positif. Si seulement il y avait plus de stabilité ! Le journalisme mène trop à tout. Et je pense justement qu'on en sort trop !

[115]

J.B. Considérons la réalité bien à part de l'éditorial. C'est généralement plus brossé, plus mûri, plus analytique aussi, concocté dans le silence dont sont privés les cueilleurs de nouvelles.

G. B. Nous avons eu par le passé de grands éditorialistes, des vedettes presque. Ces gens émergeaient d'autant plus que la qualité moyenne de leur entourage était inférieure de beaucoup à celle d'aujourd'hui. Nous ne connaîtrons plus guère de ces éditorialistes dont la signature tenait du sceau royal. Parlant de signature, la signature de l'éditorial, puis de toute la nouvelle, porte à soigner davantage le produit. C'est une pratique relativement récente. Elle facilite aussi un certain exhibitionnisme. Des directeurs d'information ont beaucoup de difficulté à contenir le journaliste à l'intérieur dans son rôle de reporter. C'est tentant pour un reporter de truffer son papier de considérations ouvertement éditoriales.

J.B. Quand vos papiers s'assimilent à des éditoriaux, c'est moins pour promouvoir une cause en particulier que pour exprimer un souci que "ça 
marche ", qu'on soit en fait "plus pratique "! Une coquetterie de théoricien?

G.B. M'adressant à des gens qui se font forts d'être des hommes d'action, je me soucie au plus haut point d'être aussi pragmatique qu'eux, je l'ai déjà dit. Je les mets en face de leur propre dialectique dans la poursuite de leurs objectifs déclarés. Je tente de discerner les aboutissants de leur option, les choix possibles qu'elle permet, ses risques aussi qu'il est préférable de prévoir que de subir après coup. Il ne s'agit pas que d'empêcher les empoignades politiques, mais de clarifier les zones imprécises des options en présence. Et il m'apparaît souvent nécessaire de rappeler les règles du jeu et une certaine morale de la lutte aux combattants, pour ne pas parler d'élégance dans le combat.

J.B. L'article du 31 décembre 1979, Avant le tournoi des [116] frères ennemis, illustrait bien cette préoccupation qui revient souvent dans vos textes.

G.B. Je disais en gros aux deux clans, à quelques mois du référendum : n'oubliez pas, dans cette lutte provisoire, que vous êtes dans le même bateau, liés par cela même qui vous oppose, adversaires mais complémentaires à la fois. N'oubliez pas entre vous cette solidarité plus profonde qui vous lie. Vous ne pouvez pas le dire, ce serait mal vu des militants et de la nuée de vos partisans. Mais si vous voulez réellement faire avancer les choses, ne perdez pas de vue l'intérêt plus fondamental entre vous et pour lequel vous allez vous affronter. Car ce qui, à la fin, paraîtra possible, il faudra le faire ensemble si vous ne vous abîmez pas trop mutuellement.

J.B. On a parfois l'impression que vos articles sont moins destinés au public général qu'aux leaders des forces en présence. Vous considérez-vous comme une espèce de médiateur dans une grande mêlée et qui vous inquiète par ses côtés excessifs, au moins sur le plan de l'expression verbale. 
G.B. Ce serait assez juste. Les hommes au pouvoir, et ceux qui en rêvent obsessivement, ont besoin du rappel d'une conscience qui soit plus exigeante que le confort de leur bonne conscience assez élastique ! Ils ont besoin qu'on leur rappelle que leurs responsabilités dépassent la satisfaction de leurs partisans ou de leur ego, que l'adversaire n'est pas aussi noir qu'ils le prétendent, qu'un jour ils devront peut-être rechercher une coalition avec cet adversaire systématique, mais qui n'est pas un ennemi à jamais.

Je peux me permettre de dire cela. Je suis seul, une simple voix parmi d'autres. Je parle dune situation de faiblesse, qui est celle de la solitude. Je ne fais pas peur, je n'effarouche pas. Je n'ai d'autre arme que la validité morale de ma conviction. Au moins cela est dit à qui de droit. Des gens qui me lisent depuis Isocrate estiment que ça doit continuer à être dit.

\section{J.B. Vous tenant loin des pouvoirs, que savez-vous de votre influence et de votre crédibilité auprès d'eux ?}

G.B. Très peu. Les chefs de parti, c'est bien connu, ne lisent pas les journaux ; c'est non moins connu qu'ils les lisent en catimini ou que leur entourage se charge de le faire pour eux ! Quant au personnel politique en général, j'évite de le rencontrer, surtout ceux avec qui j'étais à « tu » et à « toi » avant leur entrée en politique : renouer des liens affectifs - pro ou anti - risquerait de gauchir ou d'atténuer ce que j'ai à dire. Mais je vois du monde qui voit du monde qui... Ainsi, je peux apprendre parfois que tel de mes articles a été discuté dans l'entourage d'un ministre. En d'autres occasions, quand même rarissimes, participant à des émissions d'affaires publiques avec des gens des partis, mon interlocuteur politique sait manifestement que j'ai débattu ce sujet il y a trois jours, ou trois mois, ou trois ans et me le signale. 
J.B. Prenons un cas précis : la question référendaire du 20 mai. Vous êtes peut-être celui qui a le plus spéculé là-dessus, en particulier par un long papier sur « le supplice de la question (inconnue) » 15

G.B. Elle n'allait pas complètement dans le sens de ce que j'avais proposé, mais elle renfermait l'essentiel de mes exigences. Je n'acceptais pas ses éléments explicatifs, le préambule. Décidément, j'ai développé une allergie aux préambules !

J.B. Vous faites allusion à votre lutte de l'été 1980 contre le projet de Trudeau d'un Préambule de la Charte : « Nous, le peuple du Canada... ». Puis-je vous demander si l'espoir d'un feed-back est votre principale incitation à écrire?

G.B. Absolument pas ! J'en sais moins sur l'influence possible de mes articles que la boîte à lettres du journal qui [118] me publie. Une préoccupation de cet ordre serait vite désillusionnée ! Il faut adopter l'attitude fataliste et un peu détachée du « Ça donnera ce que ça donnera ! » Rien : tant pis ; quelque chose, tant mieux ! Il faut continuer à le faire. À cet égard, je jouis d'une sérénité parfaitement fataliste.

Je ne vous apprends pas que l'influence est une notion très fluide. Quand on en a les moyens analytiques pour un cas précis, on n'arrive à la mesurer que par vagues approximations ou proportionnalités du genre : le facteur A a eu plus d'importance que le facteur $\mathrm{B}$ pour produire le résultat $\mathrm{C}$. Pour répondre à votre question, la portée d'une influence éventuelle ne peut guère s'évaluer que par une impression dont certains indices, connus accidentellement, peuvent signaler qu'elle a quelque fondement. Si l'on se donne la peine d'écrire c'est qu'on espère au fond que ça ne sera pas complètement perdu. Il faut faire «comme si... » et ne pas se poser d'autres questions. Et puis, quelle « influence » ? Sur une question particu-

15 Ce Jour-là... le Référendum, Montréal, Éditions Quinze, 1978, chapitre 25. 
lière, ponctuelle avec l'espoir d'un effet enregistrable, on est mieux de laisser tomber! Mais les influences, en ce qu'elles ont d'immanent et de diffus, qui finissent par créer des ambiances générales de critique ou d'opposition, il faut y croire. C'est une question qu'on peut relier à ce que les anthropologues appellent les « représentations collectives ", et qui, toutes collectives qu'elles soient, sont souvent parlées individuellement... L'idée qui me viendrait ensuite à l'esprit serait celle de la gratuité de l'acte. Mais en seconde réflexion, je me demande si ce serait tellement changer de sujet...

\section{J.B. Cette attitude renvoie à une certaine conception du rôle de l'intellec-} tuel.

G.B. Nous n'avons pas à réclamer de statut particulier du fait que nous sommes intellectuels, et que nous « causons » plus que d'autres ! Malgré tout, l'intellectuel dans ce pays est assez estimé, ce qui est souvent le cas dans de petites sociétés. Ça peut aller jusqu'au vedettariat, ce qui est bien la facilité la plus périlleuse [119] pour des gens de notre espèce. Étant la conscience critique de pouvoirs, les intellectuels sont susceptibles de déplaire. Leurs idées les placent un peu à l'écart ou en avant de la majorité aussi : ils sont plus à gauche, en ce qu'ils sont d'éternels insatisfaits du cours des choses et qu'ils prônent des réformes profondes et surtout rapides ! Les pouvoirs publics absorbent assez aisément leurs critiques : ils sont plus sensibles au poids du nombre (sondages, élections) qu'à la puissance de l'idée. Notre influence, toujours diffuse quand elle est réelle, reste corrélative à l'estime que le public nous accorde. Mais cette estime est assez paradoxale. Voyez le cas de la France où leur prestige est très grand. À la question d'un sondage du printemps 80, demandant aux gens si leurs opinions influençaient la leur propre, la réponse massive a été "Non ». D'ailleurs, si notre influence était si grande, l'évolution générale suivrait le rythme de nos idées, parfois novatrices mais aussi parfois conservatrices. Car l'on a tendance à sous-estimer ou à ignorer la réalité des intellectuels conservateurs : les pouvoirs et les partis les recrutent à titre d'experts, de consultants. D'autres peuvent servir de caution ou de référents. Ce serait trop long de poser cette question avec la clarté nécessaire. 
J.B. Il vous arrive d'interpeller les chefs de parti ou de gouvernement dans des lettres publiques. Je pense aussi à votre conclusion pressante de Ce jour-là... le Référendum : "À Pierre et à René pendant qu'il en est encore temps."

G.B. D'abord, je ne multiplie pas l'interpellation directe ou la forme épistolaire. C'est très accidentel chez-moi. Mais les deux Lettres auxquelles vous faites allusion, parues dans le Magazine Maclean, ont été écrites dans des circonstances exceptionnelles. La première à Pierre Elliott Trudeau, tout de suite après la crise d'octobre, le mettait en cause en tant que détenteur des grands pouvoirs et évoquait un ensemble de valeurs fondamentales que je savais qu'il partageait. Il fallait prendre ce tour personnel. Je l'ai fait peu de [120] temps après pour René Lévesque, alors chef du P.Q. Il traversait une période dure : sa formation était dans l'opposition ; lui-même se morfondait en-dehors du parlement ; l'avenir semblait bouché. Des rumeurs concordantes disaient qu'il jonglait avec l'idée de se retirer. J'ai cru bon de lui rappeler son destin politique, son caractère d'indispensabilité à la cause et aux valeurs qu'il incarnait. Je l'exhortais à rester à son poste. Là aussi, il fallait prendre ce tour personnel. Quant à la finale de Ce jour-là... je l'ai écrite entre Noël et le jour de l'an 1977-78, sous le coup d'une angoisse contrôlée qui, à vrai dire, remonte à certains jours...

\section{J.B. On a parfois l'impression que vous jouez au précepteur.}

G.B. C'est, sans doute, que je ne m'exprime pas toujours comme il faut. Tout à l'opposé du langage d'un précepteur, le mien ne dit pas : « Vous devriez faire telle chose, parce que je le dis. » Ce discours déontologique n'est pas celui que j'adopte. Je dis plutôt : « De votre objectif ou de votre action découle ou va découler tels effets que vous ne semblez pas voir. Est-ce que vous voulez ces conséquences-là ? Il est encore temps de vous raviser, etc. » Un précepteur a le souci d'enseigner et de se faire obéir ! Ces gens du pouvoir, surtout en haut de l'échelle, pris dans des horaires impossibles qui grugent tout leur temps, ayant à régler dix ou vingt problèmes en même temps, n'attendent pas l'opinion de qui que ce soit de l'extérieur avant de décider ou... de ne pas décider - ce qui est une décision. De toute façon, 
l'analyse d'un esprit indépendant, à supposer qu'ils aient le temps d'en prendre connaissance, aura toujours moins de poids à priori que cet indice tout puissant des sondages ou même que la rumeur publique. Mais il reste que les hommes politiques ont aussi besoin d'une certaine stabilité psycho-intellectuelle. Et c'est parce que j'appellerais « les interstices dans leurs blocs de certitudes » qu'on peut parfois les atteindre...

\section{J.B. Ce n'est plus alors strictement l'homme d'opposition que vous pré- tendez être.}

G. B. N'est-ce pas aussi une forme d'opposition que ces rappels à la conscience latente, éventuellement active ? Il y a aussi la possibilité de renforcer parfois le groupe des opposants au sein même des formations politiques. Vous savez, les dirigeants doivent combiner des aptitudes de dompteurs et des instincts de prédicateurs. Il leur faut faire marcher tout un monde ensemble, et quelle faune c'est ! Les associés politiques sont aussi des rivaux en puissance et parfois en acte ! Il arrive que les points de vue que j'exprime dans mes articles soient invoqués par des gens qui veulent contester le point de vue officiel de leur parti. Dans un caucus ou une réunion, ils peuvent dire : «Même Untel pense cela sur telle question », pour accorder un supplément de poids à leur argumentation contre l'opinion officielle. En tout cas, c'est l'écho le plus fréquent, même s'il ne l'est pas tellement, qu'on peut avoir d'une certaine influence de ses écrits.

J.B. Si des opposants à la ligne de leur parti y trouvent quelquefois leur compte, vos analyses ont aussi le don d'agacer à peu près tout le monde !

G.B. Possible. Je n'écris pas pour agacer. Mais je ne m'abstiens pas d'écrire par souci de ne pas agacer. J'embête surtout les gens des partis qui sont encore capables d'une réflexion autonome. Personnellement, je serais proprement insupportable au sein d'une formation, à souvent rappeler les exigences de la ligne du parti. Les gens de mon espèce ne moisissent pas longtemps dans les partis ou, 
alors, ils s'en emparent ! Nous avons le privilège - que d'aucuns trouveraient douteux - d'avoir deux fabricants et manipulateurs d'idées comme premiers ministres.

\section{J.B. Est-ce qu'on ne tombe pas depuis tout à l'heure dans l'image de l'universitaire comme analyste froid de situations humaines ?}

G.B. Alors ça s'appliquerait assez mal à mon cas ! J'écris aussi pour libérer mes rares angoisses, pour évacuer mes plus fréquentes inquiétudes. Plutôt que de mourir avec, sans que ça ne sorte jamais! Appelez cela de l'écriture thérapeutique, si vous voulez...

\section{J.B. Comment vos collègues universitaires voient-ils votre activité journa- listique quand ils vous en parlent?}

G.B. Vous avez bien dit « quand ils vous en parlent ». La coutume, c'est qu'ils n'en parlent pas. Vous avez déjà entendu l'expression de « conspiration du silence ». Nous manquons de simplicité. Nous gardons nos accords ou désaccords en nous. Ou notre indifférence... Tenez, il m'arrive d'aimer tel article, telle recherche ou tel livre d'un collègue, et de lui dire lorsque je le rencontre. Bouche bée, il ne sait pas comment répondre, tellement c'est inhabituel! Maintenant, je fais attention... Il ne faut tout de même pas embêter un collègue pour ce qu'il fait de bon !

\section{J.B. Est-ce que cela tient à la psychologie de l'universitaire ?}

G.B. Cela tient à toute psychologie humaine. Or, comme les universitaires sont des hommes... Or, comme les journalistes sont des hommes... Or, comme... etc. Mais je ne voudrais pas laisser l'impression que j'ai enregistré de nets sentiments négatifs à l'égard de mon activité journalistique à l'université. Si c'était le 
cas, je m'en serais rendu compte. Si je ne m'en rends pas compte, c'est peut-être que je ne sais pas. Mais ce que je ne sais pas, je n'ai pas à en tenir compte...

\section{J.B. Cette activité publique a l'heur de vous faire connaître à l'extérieur des murs de l'université aussi.}

G.B. De là peut naître une autre ambiguïté. Sa cote d'estime universitaire peut s'en trouver rehaussée par une [123] certaine notoriété publique que procure le journalisme. Puisqu'il s'agit de cote universitaire ou scientifique, il serait indiqué qu'elle se fasse par des critères proprement universitaires : enseignement, recherche, publications, sans prendre en compte d'abord cette petite notoriété. Remarquez que ça peut aussi agir dans l'autre sens. L'ambiguïté peut aller dans les deux directions.

\section{J.B. Cela vous gêne?}

G.B. Non, pas vraiment. Mais il y a quelque chose de factice dans le fait d'une autorité universitaire qui accorde une importance parfois exagérée à ce que des membres du corps professoral s'exhibent à la télé, à la radio ou dans les journaux.

\section{J.B. D'autant plus que c'est généralement une activité seconde.}

G.B. Seconde ne veut pas dire secondaire, au sens où l'on parle de « résidence secondaire ». Tout ce que je dis depuis le début de ces entretiens montre que mon activité journalistique n'est pas secondaire et ne met pas en cause des valeurs secondaires. J'y transpose en partie mon mode de formation. C'est aussi une forme de devoir civique du citoyen de plein droit. Pas secondaire, non plus, parce que je ne peux pas m'en passer. Mais c'est second. Dans l'ensemble de ma vie de travail, mes recherches de fond ont pris beaucoup plus de temps et d'énergie que mes incursions dans le journalisme. Enfin, tout cela s'inscrit aussi dans une cohérence 
générale de vie. J'ai comme métier d'analyser la vie politique : penser, comprendre nos sociétés en tant que politiques. Ce n'est pas impur, de temps en temps, de donner de soi hors du circuit universitaire, qui est finalement assez court.

J.B. Cette carrière journalistique très tôt envisagée, puis [124] mise de côté partiellement sinon totalement, pourrait-elle devenir une carrière de fin de vie?

G.B. Je ne suis pas encore à la veille d'une retraite. J'espère avoir encore quelques bonnes années devant moi. La question n'est pas incongrue dans la mesure où, à la parution de ces entretiens qui sont le bilan provisoire de mes travaux, j'aurai pris le tournant de la soixantaine. C'est un âge où il faut réfléchir à la façon de finir sa vie active. En effet, pourquoi ne pratiquerais-je pas, à l'âge de la retraite active, le métier que les circonstances de la vie auront empêché plus jeune ? Je transformerais simplement en activité première une collaboration à laquelle je consens périodiquement depuis vingt-cinq ans comme activité seconde. Chose certaine, je ferais plutôt ça que d'écrire mes mémoires! Les présents entretiens vont suffire pour l'impudeur...

\section{J.B. Votre nom a circulé parmi les candidats à la succession de Ryan au Devoir. Excluriez-vous une direction de journal ?}

G.B. Oui, parce que c'est un tue-monde ! Il était un peu normal, à cause de mes longues accointances avec ce journal, que mon nom sorte parmi une bonne demi-douzaine d'autres noms assez plausibles. Pour caractériser ce type de collaboration externe quelque peu suivi, Ryan se servait de l'expression assez pittoresque de «fréquenter Le Devoir ». Je suis celui qui aura eu les plus longues fréquentations, mais... sans jamais l'idée de mariage de part et d'autre. 
J.B. Le caractère plausible d'une candidature à la direction du Devoir avait tout de même quelque chose de flatteur.

G.B. Si vous voulez. Le Devoir étant plus qu'un journal, son directeur est plus qu'un directeur : il devient une [125] personnalité sociale et politique de premier plan dans notre société. Heureusement, après un intérim assez inquiétant qui aura duré trois ans, Le Devoir est maintenant assuré d'être entre de bonnes mains. 
[127]

$\begin{gathered}\text { GÉRARD BERGERON. } \\ \text { de l'autre côté de l'action. }\end{gathered}$
$5^{\mathrm{e}}$ entretien
L'ESSA YISTE
«... l'État, toujours Lui ! »

$\underline{\text { Retour à la table des matières }}$ 
J. B. Si l'on met à part la dizaine d'ouvrages que votre bibliographie classe dans l'une ou l'autre catégorie de la « théorie politique » ou des « études de conjonctures » (dont il a été, respectivement, question lors des deux entretiens précédents), on trouve une troisième catégorie portant le titre d' « essais » et qui semble un peu résiduelle. On y trouve en particulier votre Canada français après deux siècles de patience, publié à Paris en 1967, qui est la pièce la plus soutenue dans le genre essai historique. Mais dans vos ouvrages des dernières années comme Incertitudes d'un certain pays (1979) ou L'État du Québec en devenir (1980), on peut lire une série d'essais plus courts et portant sur toute une gamme de sujets : sur l'histoire de la politique extérieure du Canada, sur celle de nos partis à l'un ou l'autre niveau provincial ou fédéral, sur la réforme constitutionnelle bien sûr et l'histoire mouvementée des relations fédérales - provinciales, sur la politique québécoise à l'époque de Duplessis, sur l'évolution du système judiciaire québécois des vingt dernières années. On s'aperçoit encore que l'ouvrage, qui suit immédiatement celui où vous semblez vous être amusé à portraiturer une quarantaine de nos politiciens, est une histoire de la guerre froide jusqu'à 1970, et qui est à ce titre le plus grave de vos livres. Enfin l'on prendra en compte toute une production, non publiée mais rendue publique sur les ondes de RadioCanada pendant dix ans, qui portait sur les phénomènes de la chanson, aussi bien québécoise que canadienne, ainsi que sur le star system au cinéma sous le titre plus évocateur de « Mythes et monstres sacrés ».

[130] De tout cela, de toutes ces écritures que vous avez menées parallèlement, il se dégage une impression de dispersion. Vous comprendrez qu'on puisse se poser la question de l'unité de tout cela, d'autant que vous m'avez avoué être tenté par d'autres sujets et même par d'autres genres (de fiction, peut-être ?). Quand vous arrêterez-vous ? Ou plutôt, êtes-vous sûr de bien faire en élargissant encore le champ de vos sujets d'essais?

G.B. Ouf ! Cette nomenclature même a quelque chose disons d'incriminant, sinon d'accusatoire ! Mettons-nous d'abord d'accord sur un point : la diversité, pas 
plus que la prolixité, n'est une qualité en soi. C'est à voir. On va encore laisser de côté la contradiction généraliste-spécialiste dont nous avons parlé à l'entretien consacré au théoricien. Disons d'abord qu'à chacun des niveaux de mon oeuvre, il y a un livre plus important que les autres qui se trouvent alors à le prolonger ou à en être des appoints ou des ajouts. Ainsi pour la théorie politique, c'est Fonctionnement de l'État, le tout premier dont le reste allait découler. Pour le niveau des conjonctures politiques, c'est, au contraire mais tout naturellement, le dernier en date qui paraîtra à l'automne sous le titre de Syndrome québécois et mal canadien. À l'enseigne des essais, c'est l'ouvrage que vous avez d'abord retenu Le Canada français après deux siècles de patience, publié en 1967 à Paris. C'est un essai synthétique constituant un dossier de base sur le cas du Québec, tel qu'on pouvait en voir les éléments en 1966, il y a une quinzaine d'années.

\section{J.B. C'était donc dans la foulée de votre premier ouvrage théorique et du premier recueil d'articles d'Isocrate que Gérald Godin vous avait demandé et publié. Mais pourquoi l'avoir publié à Paris et à ce moment-là ?}

G.B. Parce que les éditions du Seuil me l'avaient demandé depuis une couple d'années et qu'ils avaient insisté pour que l'ouvrage soit publié pour l'année du centenaire [131] de la Confédération, coïncidant avec la tenue de l'Expo à laquelle devaient assister beaucoup d'étrangers et de Français notamment. Il y avait aussi que nos grands cousins nous redécouvraient à l'époque de la Révolution tranquille et que la Délégation du Québec avait pignon sur rue à Paris depuis 1961. Était annoncée aussi la visite du général de Gaulle pendant cette année de grandes festivités, sans que personne ne se doutât (sauf peut-être le général lui-même et ce n'est pas sûr...) de l'immense tapage que cette visite allait entraîner.

C'est donc un livre que j'ai écrit en commande, mais ce fut le premier et le dernier. Je vous ai déjà dit que je n'ai rien contre les commandites, sauf que je n'en fais pas. Je n'aurais pas accepté la demande du Seuil si je n'avais pas déjà porté ce livre en moi, sachant bien que j'écrivais un tel livre à point nommé, ne fut-ce que pour éclairer ma propre lanterne en rassemblant en un essai synthétique les pièces du dossier. Il y avait aussi que le contexte des retrouvailles FranceQuébec et de la prochaine exposition de Montréal était une circonstance émi- 
nemment favorable. En outre, j'éprouvais un besoin profond de me dépayser des laborieuses constructions théoriques sur l'État en général. Allant en France régulièrement depuis 1947 et y ayant séjourné plusieurs années pour mes études et mes recherches, j'avais pu mesurer le degré d'ignorance et de curiosité bienveillante que manifestaient non seulement les Français en général mais même l'intelligentsia parisienne. Pourquoi ne pas leur rendre ce service, en même temps que je m'en rendais un, en écrivant ce livre que, je le répète, je me sentais en état de nécessité d'écrire à ce moment-là ?

\section{J.B. C'est vrai que l'information sur le Québec ou les affaires canadiennes étaient plutôt rare à l'époque.}

G.B. Claude Julien, journaliste au Monde dont il est censé devenir le directeur à la fin de 1982 par voie d'élection de la société de ses journalistes, avait été le premier journaliste français d'importance à faire de "grands reportages » sur le Canada dès 1951. J'ouvre une [132] parenthèse pour rappeler qu'au sortir de la guerre il était entré en contact avec des jeunes d'ici, engagés comme lui dans le mouvement d'action catholique, un Gérard Pelletier, un Guy Rocher et plusieurs autres. Il avait aussi entretenu des contacts avec le groupe de Cité Libre, Pelletier et naturellement Trudeau, son penseur politique officieux et très influent dans ce contexte d'époque.

Claude Julien, donc, avait publié en 1965 Le Canada, dernière chance de l'Europe. Avec un pareil titre, emphatique et presque tonitruant, l'auteur se plaçait d'un point de vue naturellement européen et laissait percer quelque antiaméricanisme qui sera encore plus accusé dans d'autres ouvrages. Le côté un peu embêtant de l'ouvrage était que « nous » - Canadiens - étions vu sous un jour plutôt instrumental à l'indépendance européenne devant l'hégémonisme américain en économie et en sécurité internationales. Quant à «nous » - Québécois - nous étions vus en profonde sympathie culturelle mais selon l'orthodoxie fédéraliste de Cité Libre première manière, c'est-à-dire dans une perspective proprement trudeauiste. Voilà pour le livre le plus important nous concernant; il n'y avait guère rien eu d'autre sur cette question depuis le Canada, puissance internationale (autre titre emphatique) qu'André Siegfried avait fait publier avant la guerre. Mon 
Canada français après deux siècles de patience tombait pile pour satisfaire la curiosité naissante des Français à ce moment.

\section{J.B. C'était un bon titre.}

G.B. Oui, je pense, et dont il faut accorder le crédit à Jean Lacouture, directeur de la collection où mon livre parut. Vous aurez noté que « les deux siècles de patience » évoquaient une étude qui tiendrait compte de la perspective historique, ces deux cents ans de notre séparation d'avec la mère-patrie. En outre, on ne disait pas encore le Québec, mais le Canada ou le Canada français. Mais la mode allait changer très vite et surtout à partir de la fondation du Parti québécois en 1968. Et quand quelques années plus tard, Marcel [133] Rioux fera paraître un autre livre chez Seghers ce sera sous le titre de La Question du Québec.

\section{J.B. Dès l'avant-propos de votre livre, on retrouvait déjà votre position} médiane se refusant de s'embarquer dans l'une ou l'autre des thèses qui s'opposent encore.

G.B. J'y disais qu'il était facile d'écrire deux livres : soit sous l'angle de l'impératif de l'indépendance ou de la nécessité plus sécuritaire du cadre fédéral englobant. Je préférais écrire un troisième livre, plus difficile, car ce n'était pas ainsi que la partie allait se jouer, mais bien plutôt dans une dialectique serrée et longue entre ces deux vecteurs. C'est encore la situation quinze ans plus tard.

\section{J.B. Ce livre était d'une construction assez bizarre.}

G.B. En tout cas, assez peu courante. Il fallait jouer du triple facteur temps, espace et nombre. Il fallait amener le lecteur français à voir que le Québécois francophone lui était à la fois aussi différent que semblable. Nous jouissions à l'époque d'un fort préjugé favorable. C'était fondé sur une sympathie d'instinct,

entretenue par une curiosité ignorante et parce que j'appellerais un relent de 
« compensation historique » pour la perte de l'Algérie. Il s'agissait de le rendre familier avec cette espèce particulière de l'homo quebecensis * .

Ce que vous venez d'appeler la construction bizarre de l'ouvrage, c'était le plan en forme de sablier : un visionnement très large au départ puis se réduisant graduellement en cercles concentriques jusqu'à la saisie au centre de cet homme du Québec, puis le retour en cercles concentriques s'élargissant jusqu'au visionnement final : les Québécois dans le monde : la marche vers l'indépendance. J'ai la table des matières devant les yeux, soit : I. Le Canada dans le monde : l'espace ; II. Le Canada en Amérique du nord : le voisinage ; III. Le Canadien français dans le Canada : l'histoire ; IV. Le Québec dans le Canada [134] français : le nombre ; V. Les Québécois dans le Québec - c'était le goulot d'étranglement - l'homo quebecensis ; VI. Les Québécois dans le Canada : la Révolution tranquille ; VII. Les Québécois en Amérique du Nord : le nègre blanc; VIII. le développement final déjà dit - la marche vers l'indépendance ou le Québec dans le monde.

J.B. Vous avez oublié de rappeler que l'introduction s'intitulait d'une façon quelque peu provocante : «La plus grande réussite (involontaire) de la France ".

G.B. Elle se terminait par une citation du président de Gaulle recevant le premier ministre Pearson en $1964:$ « ... ceux des habitants de votre vaste territoire qui sont notre peuple installé au Canada ».

J.B. Vous me rappeliez avant cet entretien que le général avait manifestement lu votre livre avant de venir au Canada. Vous n'avez pas d'objection à nous en parler un peu.

G.B. Mon directeur de collection, Jean Lacouture, gaullologue lui-même, me demande lors de la publication : "Avez-vous l'intention de l'envoyer au Général ? » Même si je ne débarquais pas à Paris pour la première fois, j'avais assez

* Voir le document à la fin de ce chapitre. 
peu l'habitude de communiquer avec les grands de ce monde, pas plus avec le général-président que les autres ! « Il vous lira, insiste Lacouture. Il prépare toujours minutieusement ses dossiers et votre livre arrive à point ». Dans ces conditions, je n'avais pas d'objection à lui en envoyer un exemplaire. Le livre partit donc pour l'Élysée avec une double dédicace du Seuil et de l'auteur. Je recevrai un peu plus tard une lettre de remerciement du secrétaire-général de l'Élysée, précisant que « le Président l'avait lu et qu'il m'en remerciait ».

\section{J B. Vous en aurez d'autres preuves plus tard.}

G.B. Vous vous souviendrez qu'après le cri fameux qui allait faire le tour de la planète, de Gaulle avait raccourci son voyage et était rentré sans avoir fait un crochet à Ottawa comme prévu. À son retour précipité, il avait réuni son Conseil des Ministres en pleine nuit. Le tintamarre du «Vive le Québec libre ! » au Canada avait eu le temps de faire son tour de presse mondial et le moins qu'on puisse dire c'est qu'à Paris on était loin d'être unanime sur l'exclamation inattendue du général. À la sortie de la réunion du Conseil, un journaliste de L'Eixpress avait soutiré une indiscrétion d'un des ministres sur la teneur des propos que leur avait tenus le Président. En substance, il leur avait dit que, s'ils étaient étonnés de ses propos, c'est qu'ils ne connaissaient pas la situation qui prévalait au Canada et qu'ils feraient mieux de se renseigner en lisant Le Canada français après deux siècles de patience et Les Québécois. Ce dernier livre était constitué d'un choix d'articles de la revue Parti pris et avait paru chez Maspéro peu de temps après le mien.

À sa conférence de presse de novembre suivant (le général en donnait deux par an), constatant toujours de fortes réserves et même de l'hostilité à son endroit, tant dans la presse internationale que dans la presse française, ayant été pris à partie par toute l'opinion de gauche et ayant mis à la gêne beaucoup de gaullistes, de Gaulle sentit le besoin de justifier de long en large le cri qu'il avait lancé du haut du balcon de l'Hôtel de ville de Montréal. Il donna à la presse un véritable cours d'histoire sur les «Français du Canada » en des termes qui m'apparurent à moi, en tout cas, pas très éloignés de la présentation du chapitre de mon livre où j'avais résumé cette histoire. 


\section{J.B. Vous accordez de l'importance à ce fait.}

G.B. Admettez que c'est assez peu courant. Mais avant de vous livrer ma pensée là-dessus, je veux faire une observation d'ensemble. Je suis convaincu que de Gaulle aurait eu la même audace sans avoir lu mon livre, sans l'avoir annoté et apporté avec lui à bord du Colbert (détail qui me sera fourni par hasard et longtemps [136] après par des gens de la Délégation à Paris, qui eux-mêmes tenaient ce renseignement de personnes du cabinet de la Présidence). Il se serait renseigné ailleurs ou autrement. L'aurait-il fait de la même manière ? Comment savoir ? Ce qu'on sait plus clairement, des années plus tard et après beaucoup d'études gaulliennes, c'est que, pour un ensemble de raisons qui seraient longues à évoquer ici, le général méditait, si l'on peut dire, un coup au moment de sa visite parmi nous.

\section{J.B. Vous aurez eu tout de même un agent de publicité plutôt presti-} gieux...

G.B. Et au sens plus strict que vous ne le pensez avec cette allusion ironique. Deux jours après la rentrée du général, mon éditeur fit paraître une lucarne publicitaire en première page du Monde qui disait ceci sur quatre lignes : Pour y comprendre quelque chose... Le Canada français après deux siècles de patience... de Gérard Bergeron... Éditions du Seuil, etc. Les ventes sont reparties en flèche en ces semaines de vacances 1967. Mais il est assez curieux de voir qu'en plus longue période mon livre allait s'écouler davantage et de façon plus continue ici même au Québec.

J.B. J'ai pu constater que, vers ces années-là, votre livre servait de texte d'enseignement dans les cours d'histoire ou d'études québécoises dans les cégeps. Mais revenons aux considérations plus larges que vous avez annoncées sans les avoir développées. 
G.B. Reprenons l'expression de l'annonce du Monde: «Pour y comprendre quelque chose... » Lisons-nous pour autre chose ? Et même, écrivons-nous pour autre chose ? Surtout si l'on tient compte de tout ce qu'il faut lire avant de se mettre à écrire ! Pour ma part, j'ai l'impression de ne commencer à comprendre quelque chose que lorsque je réussis à le mettre par écrit. Cela, en tout cas, n'est jamais complètement [137] perdu de ce point de vue. Quant au résultat escompté et hors de soi-même, c'est autre chose ! La plupart du temps, les écrits d'un auteur ne font pas mouche. Ils connaissent une carrière restreinte et éphémère quand ils ne passent pas tout simplement inaperçus. On doute alors que ça vaille la peine de continuer. Est-ce raisonnable, tenant compte de la somme d'énergie humaine qu'on met dans ces travaux ?

Ce qui est arrivé avec le livre en question montrerait qu'on ne devrait pas s'arrêter à des considérations de ce genre. On ne sait jamais, même si le phénomène est rare, à quel moment une pensée, propagée par un livre ou un journal, peut rebondir de façon fort inattendue. Cela implique une grande responsabilité. Il m'arrive parfois de retrancher des phrases ou de nuancer des affirmations en me disant : "C'est ce que je crois comme vrai, mais c'est peut-être dangereux ». La noblesse de l'intellectuel tient à la fois de la nécessité et de la gratuité où il se trouve de porter un message humain, de vie. Quelle que soit la portée, faible le plus souvent, de ce message, il faut se dire que ce n'est jamais une denrée triviale. De sa nature. On peut parler ainsi sans tomber dans le maniérisme corporatif des écrivains qui aiment causer entre eux de leur « mission », de leur « témoignage ». Ils se grossissent un peu. Mais quelle corporation ne s'enfle pas quand ses membres se regardent entre eux ?

J.B. Il vient d'être question de de Gaulle. Vous m'avez déjà confié que dès votre retour d'Europe, au tout début des années 1950, vous aviez prononcé une série de causeries à Radio-Canada portant sur des portraits en parallèle d'hommes d'État. Y aurait-il aussi du Plutarque en vous ?

G.B. J'ai toujours été fasciné par les grands destins politiques et surtout quand on peut, à la façon de Plutarque, mettre leurs vies en parallèle. Dans la série en question, je l'avais fait pour Staline et Roosevelt, Churchill et Adenauer, Eisen- 
hower et de Gaulle, Tito et Peron et peut-être une couple d'autres paires dont je ne me souviens pas. Aussi, quand mon intérêt s'est confirmé [138] en politique canadienne, je n'ai jamais renié une curiosité de cet ordre.

\section{J.B. Ce fut l'occasion de votre galerie de portraits dans Ne Bougez plus !}

G.B. Ce fut aussi l'objet d'un petit divertissement à l'été 1968. Je venais de terminer deux ouvrages exigeants coup sur coup. J'en mettais un autre en chantier tout autant éprouvant. J'avais besoin d'un exutoire, de cette soupape, pour laisser passer un peu de la politique se vivant... Dans et par des hommes concrets, très individualisés, et qui en outre étaient d'ici. J'ai donc fait cette galerie de quarante de nos politiciens : vingt dans l'arène québécoise, dix-neuf dans la fédérale et le dernier était celui de Montréal qui règne encore. Il n'y avait pas que les grandes vedettes comme vous voyez, mais aussi un certain nombre de ceux qui comptaient par leur influence ou qui étaient pittoresques par leur style ou personnalité. Mais j'avais une faiblesse particulière pour quelques têtes de pipe préférées dont je faisais un peu plus que d'esquisser la binette : Lesage et Johnson, Trudeau et Stanfield, mais aussi Diefenbaker et surtout Caouette, pour ne pas oublier Drapeau et Lévesque qui sont encore aux commandes aujourd'hui, comme Trudeau luimême.

\section{J.B. Plus récemment, vous êtes revenu à ces portraits.}

G.B. Oui, il y a trois ans, lors de la course au leadership du P.L.Q., j'ai esquissé les portraits de Claude Ryan et de Raymond Garneau. Et, plus récemment l'été dernier, j'ai tenté de mettre à jour les portraits de notre quatuor : Trudeau, Lévesque, Ryan et Drapeau sous des titres qui en faisaient des « types ».

Trudeau m'apparaissait comme un «monarque restauré »après sa spectaculaire rentrée alors qu'il avait annoncé son départ après sa première défaite par Clark. Lévesque, lui, après avoir perdu son référendum [139] et avant de solliciter un nouveau mandat électoral, avait l'air d'un « président à re-plébisciter ». Ryan, je le voyais comme un "prétendant tous azimuts ». Quant à Drapeau, il continue de 
porter la livrée d'« intendant des grandeurs métropolitaines 16 ». C'est le moment de constater que, si notre politique n'est peut-être pas plus digne ou efficace qu'ailleurs, elle produit une classe de leaders tout à fait remarquable pour un peuple aussi petit. Prenez le quatuor dont nous venons de parler. Où en trouverait-on l'équivalent ? Peut-être, en cherchant bien et ce n'est pas si sûr, en France qui est tout de même un pays dix fois plus peuplé. Chacun à sa façon est une bête politique assez extraordinaire et le dernier mot de Robert Bourassa n'est pas dit. Ce seraient de beaux sujets de roman, plutôt balzaciens que proustiens... Ce n'est pas médire de la politique au Canada anglais que de constater qu'ils n'ont pas d'équivalents : les Clark, Broadbent, Davis ou Hatfield, Blakeney ou Lougheed ne semblent pas avoir la même pesanteur d'humanité.

J.B. À première vue, C'est étonnant de voir un «théoricien pur "considérer les hommes politiques comme des personnage de roman ou la vie politique elle-même comme un vaste théâtre.

G.B. Oui, la politique est le lieu d'une mise en scène constamment renouvelée, d'une action dont on connaît les acteurs, jouant leur propre personnage, et, bien sûr, les enjeux et, assez bien, les intrigues. Seul le dénouement est encore inconnu mais on sait qu'il viendra. Sans parler des entrées et surtout des sorties, vraies ou fausses... Sur le strict plan dramatique, le jeu du trio Trudeau, Lévesque, Ryan (et peut-être bientôt Bourassa) a de quoi fasciner l'amateur de théâtre. Mais la grande différence c'est que l'action est réelle, que le jeu se joue réellement. Et qu'il y aura des conséquences pour nous, spectateurs captifs, mais aussi participants un tant soit peu.

J.B. On voit ainsi pointer la double passion de votre jeunesse pour la politique et pour le théâtre.

16 Syndrome québécois et mal canadien, Les Presses de l'Université Laval, Québec, 1981, chapitres 30 et 31. 
G.B. Si j'avais plusieurs vies, j'aimerais creuser tout cela et peut-être me faire le biographe de l'une de ces têtes de pipe en y apportant, plus qu'il n'est d'usage, l'éclairage de l'analyse politique proprement dite. Je vais peut-être vous étonner en vous confiant que j'y ferais montre d'une certaine commisération pour ces hommes qui ont décidé de faire notre bonheur en partie aux dépens du leur, à cause précisément de l'énormité des tâches qui les confrontent. C'est une pensée qui me revient souvent, surtout quand je me sens en veine de les attaquer plutôt rudement. J'ai peut-être tort mais je me surprends à dire : «Que ferais-je à leur place ? Qui suis-je pour leur parler sur ce ton ? » En fait, c'est assez rare que je leur parle directement. Je parle à ceux qui les suivent avec assez peu d'esprit critique, en fait à tout le monde, pour essayer de comprendre ce qu'ils font ou ne font pas... Vous voyez que cet esprit de commisération ne sombre pas dans l'indulgence. Et puis, par dessus tout, ce sont eux qui ont choisi d'être là. Et qui l'ont voulu... Et combien!

\section{J.B. À priori c'est surprenant de voir un théoricien qui, par définition,} cherche à expliquer les grands processus, les mécanismes des structures, etc. porter autant d'attention au rôle de l'homme politique individuel.

G. B. C'est peut-être, non pas en dépit de, mais précisément à cause de cette obédience à la théorie que je sens le besoin de m'y arrêter. Je termine La gouverne politique en affirmant qu'aucune théorie politique ne peut prévoir l'apparition de l'homme politique qui vient accélérer ou renverser les situations collectives de notre existence humaine. En effet, cet homme concret, individualisé, entouré de proches, daté par statut d'état-civil, etc., il est absent de nos travaux de sociologie politique. C'est le domaine des historiens une fois que tout est joué, c'est-à-dire qu'il est trop tard [141] pour nous du moins. Ou c'est celui des journalistes alors que, j'ai envie de le dire, il est trop tôt pour bien connaître notre homme. En sociologie politique, on consacre parfois un chapitre au leadership, aux types des leaders en en faisant, une fois de plus, une fonction et un rôle particuliers, et non en présentant le vécu extraordinaire et particularisé des acteurs de cette dramaturgie très spéciale. L'homme est absent de notre science politique. Nous n'en avons que 
pour les grandes structures et les grosses machines : fonctions et processus, structures et institutions, idéologies et doctrines, groupes et partis. L'homme-citoyen est fondu dans le nombre de nos analyses ; l'homme-leader à quelque niveau (j'allais ajouter : " de son incompétence », selon le célèbre principe de Peter) est estompé par l'opération des grosses machines dont je viens de parler.

Ma partie théoricienne constate le vide sur cette question ; ma partie journalistique voit comment il est un peu possible d'y pourvoir dans le vif de l'actualité : ce que je peux consentir à l'essayiste, entre les deux, me suggère à l'occasion d'esquisser ces petits portraits d'hommes politiques dont nous venons de parler. Je suis littéralement fasciné par l'homme politique. La fonction politique doit être remplie. Pourquoi par ceux-là et non pas par d'autres ? Et quelle complexion particulière cela prend pour exercer ce fichu métier ! Vous voyez, mon intérêt est de fond à la question. Il ne découle pas seulement du fait qu'une grande partie des fortes personnalités de ma génération ont fini par «plonger » - comme l'on dit - et par se retrouver dans l'une ou l'autre de nos formations politiques.

\section{J.B. N'avez-vous pas le projet de théoriser un peu plus complètement là- dessus?}

G.B. « Théoriser », c'est beaucoup dire. Je viens de rappeler que la théorie n'a guère de choses à dire sur la question. Même s'il est vrai que changer telle tête et c'est une autre politique qui se fait, c'est une autre histoire qui s'écrit, je ne serais pas tenté de reprendre la théorie de Carlyle selon laquelle l'histoire est une [142] succession de grands destins politiques, de grands hommes. Cette conception d'une «histoire-grands hommes» n'est pas plus défendable que celle de l'« histoire-batailles. » Mais j'avoue que me tenterait assez une espèce de caractérologie des hommes politiques : l'arriviste, le dominateur, le penseur, le rebelle, le mal-aimé, le prématuré, le mythomane et, bien sûr, l'incapable dont les modèles ne manqueraient pas! Pour mieux les camper, on pourrait recourir à des types de mythologies anciennes comme l'avaient fait, du reste, Shakespeare et Racine dans leurs si fortes dramaturgies politiques. Très tôt m'ont intéressé les auteurs dont l'oeuvre présentait les actions d'hommes fortement individués sur une toile de 
fond d'histoire collective : bien avant Soljenitsyne et autres dissidents, les Malaparte, Malraux, Koestler, Unamuno, Gheorgiu, Camus, etc.

J.B. C'est tout de même curieux de voir se côtoyer dans votre bibliographie des titres aussi disparates que Fonctionnement de l'État et $\mathrm{Ne}$ bougez plus!

G.B. Je me souviendrai toujours de cet étudiant, curieux et respectueux, qui monta un jour à ma tribune, suivi de sa petite amie non moins timide, pour me demander si je ne connaissais pas l'autre Gérard Bergeron qui avait écrit Ne bougez plus!

\section{J.B. D'où venait le titre en hold up d'un film western ?}

G.B. De Jacques Hébert, l'éditeur, qui était fier d'avoir trouvé cette expression du langage de photographe pour une petite collection de portraits. Il lui fallait un titre percutant. Quelques-unes de ces personnalités ont disparu depuis. Il est arrivé que des journaux, à l'occasion de leur décès, aient reproduit des portraits que j'en avait faits en mentionnant la référence, bien entendu. Ce titre de $\mathrm{Ne}$ bougez plus avait quelque chose de macabre dans les circonstances. Je n'y avais été pour rien.

J.B. Passons à du plus léger. Votre production la moins connue, c'est sans doute celle du scripteur de textes radiophoniques sur le phénomène de la chanson française, y compris québécoise, et sur les Mythes et monstres sacrés du cinéma selon le titre d'une série. Comment tout cela a commencé ?

G.B. Avant de vous le dire, j'aimerais vous faire remarquer qu'il y a une certaine parenté, disons « caractérologique », entre mon intérêt pour les leaders politiques solitaires (ce qui veut dire qu'ils sont « au sommet... ») et les vedettes du 
music hall, elles aussi fort solitaires. Cela mériterait peut-être un brin de développement tout à l'heure.

Comment cela a commencé ? Par une rencontre avec Paul Le Gendre, réalisateur à Radio-Canada et ancien copain d'université. Préparant la formule radiophonique du breakfast club de «Chez Miville » qui allait durer une quinzaine d'années, il était à la recherche de scripteurs. À une première rencontre de programmation, je m'y retrouve avec des scripteurs déjà chevronnés dans le genre de l'humour : Fernand Séguin, André Roche, Carl Dubuc, Miville Couture lui-même, Raymond Laplante et celui qui allait devenir le plus célèbre de tous, René Lévesque, qui devait prendre la responsabilité d'une chronique quotidienne d'actualité. Je précise la date : 1956, peu de temps avant que celui-ci ne lance son célèbre Point de mire à la télévision, cette superbe réussite du genre qui allait répéter ce qu'en d'autres circonstances Louis Francoeur avait fait pendant la guerre avec $\mathrm{La}$ situation ce soir. Les gens d'un certain âge s'en souviendront.

Revenant à Chez Miville, je me suis laissé prendre au jeu. Après trois mois de petits billets sur la politique internationale j'abandonnais, ne pouvant de Québec assister aux réunions de programme. Et l'émission prit assez tôt un autre style qui allait faire son succès pendant toutes ces années. Restait qu'un scripteur du dimanche était né dans la ville de Québec, où il y en avait fort peu. L'heure étant à la décentralisation à Radio-Canada (sous le coup, en passant, de la télévision aussi envahissante que jeune), Marc Thibault, directeur des programmes à la radio, m'accorde une [144] série que j'intitule Le Coin des pères (en variante au célèbre «Children's Corner » de Debussy). C'était un assemblage de textes variés, se voulant politico-humoristiques et y réussissant parfois, à l'intention des pères de famille qui s'embêtaient à la maison le samedi matin. Le script était truffé de chansons pour souligner des points de chute, formule qui avait été illustrée des années auparavant par les très populaires dialogues de Jovette Bernier et de Jacques Desbaillets. Après deux ans, la formule changea du tout au tout. M'étant familiarisé avec ce répertoire de chansons spirituelles ou humoristiques et à vrai dire découvrant la chanson intelligente de ces années-là, j'eus l'idée de faire une espèce de petite sociologie de la chanson (je dis « petite », pour que l'expression ne paraisse pas ampoulée), sous le titre de Rien qu'une chanson, ce qui est le titre 
même d'une chanson de Trenet. C'était un divertissement se voulant de bonne compagnie, mais nullement prétentieux et encore moins « savant ».

Il faut rappeler que c'était, en ces années 1950, l'âge d'or de la chanson française : les frères Jacques, Mouloudji, Léo Ferré, notre Félix et Brassens, Catherine Sauvage, Juliette Gréco, Trenet et Piaf toujours là, et Yves Montand, ce standard de perfection qui dure encore après s'être fait une réputation internationale au cinéma, et tous les autres qui n'allaient pas tarder, Béart, Brel, etc. - J'étais frappé par la qualité littéraire des textes et m'attachais en particulier à leurs grands thèmes récurrents : l'amitié, l'amour, l'argent, le succès, la mort bien entendu, mais aussi des thèmes plus sociaux, comme la fraternité, la solidarité, le refus de la guerre, notre petite boule dans le cosmos, etc. Je me souviens d'avoir fait des émissions sur l'atome ou l'énergie atomique. Bref, je me familiarisais avec cet univers de sons qui accompagnait les heures creuses de la journée ou les voyages en automobile, qui suivaient ou précédaient les lectures de bulletins de nouvelles.

Puis la formule évoluera en s'étendant sous le titre de Le petit dictionnaire de la chanson, émission qui allait durer cinq ans pour parcourir tout l'alphabet de A (Affection, Amitié, Marcel Amont, Amour, Argent, Michèle Arnauld...) jusqu'à Z (Rica Zarai et... Zut !). Il y aura d'autres séries plus courtes comme En France [145] tout finit par des chansons, une petite histoire de France par les chansons, à partir des troubadours et des croisades jusqu'aux chansons politiques sous de Gaulle. Et aussi, une autre que je fis avec un intérêt particulier puisqu'elle me permit de me recycler dans l'histoire de la littérature poétique. C'était intitulé : « ... de la musique le long de mes vers». Il faut garder les guillemets puisque c'était une formule tronquée de l'expression de Victor Hugo : « Défense de déposer de la musique le long de mes vers », rappelée au début de chaque émission. Léo Ferré avait été l'initiateur du genre avec son « Pauvre Rutebeuf ». Il s'agissait de retracer dans la poésie française les textes qui avaient, pour la plupart, été mis en musique à l'époque contemporaine. J'y fis des découvertes du tonnerre, par exemple que le grave Montesquieu avait fait des chansons lestes, ce qui était l'occasion de rappeler qu'il était aussi l'auteur des Lettres persanes. 


\section{J.B. Au tournant des années 1960, c'est alors que la chanson québécoise va s'imposer.}

G.B. Oui, et vers 1963-64, je lance une série Le Québec chante. Prévue pour quelques mois, elle durera une année entière, tant la matière intéressante devenait abondante. Je partais des origines, je m'efforçais de caractériser les époques et les genres, je m'entendais spécialement à détecter des chansons à contenu ou allusions sociales ou politiques. Je me souviens avoir décrété que la chanson de Raymond Lévesque, «Quand les hommes vivront d'amour », d'abord endisquée par Eddie Constantine, était un chef-d'oeuvre de la chanson universelle, puis que Vigneault avait «du génie ». Je crois ne vous avoir pas dit que je n'interprétais pas mes textes qui étaient dits par de comédiens ou des annonceurs à Québec. J'avais la responsabilité entière, à part la réalisation, du script, des sujets, de la discographie.

\section{J.B. C'est dans ces circonstances que vous avez rencontré Vigneault, alors inconnu.}

G.B. Cela se passait en 1959, je crois. Un jour que je préparais ma discographie à Radio-Canada, voici que surgit un type à l'allure assez bizarre, nez d'aigle et sans voix, mais avec quels yeux! Il se présente : «Moi aussi je suis professeur. Mais je fais des chansons. C.B.V. vient de passer deux de mes chansons interprétées par Jacques Labrecque sur 45 tours. » " Jos Montferrand » était l'une d'elles. Je garde contact avec lui. Entre-temps, je lis son oeuvre poétique, déjà imposante. Je suis époustouflé par l'originalité et la vitalité de cette poésie où passait la personnalité débordante de son auteur. Je serais intarissable sur cette oeuvre. D'ailleurs, vous connaissez le reste. Nous avons baptisé son premier microsillon chez moi avec un petit groupe d'amis. Mais auparavant, il avait décidé d'être son propre interprète. Il n'était pas évident qu'il serait «le monstre sacré » que je pressentais et qu'il est devenu. 
À l'été 1960, il décida donc de se lancer sur scène dans un cabaret improvisé, la Boite aux Chansons, dans le vieux Québec. Il y régnait une ambiance inouïe, chaude de communication fervente et, il faut le dire, aussi de connivence ! Ce soir du 5 août 1960, après son tour de chant d'une extraordinaire qualité de présence, mais qui était discutable professionnellement, il me demande : "Qu'en pensestu ? » - Après ce qu'il venait de nous présenter, ce ne pouvait être que l'un ou l'autre de ces deux prédictions : ou le fiasco le plus total, sombrant dans le ridicule, ou un succès boeuf qu'il ne pouvait même pas imaginer et qui dépasserait tout ce qui s'était vu dans le genre! Cette anecdote est racontée par Aline Robitaille, son premier critique biographe, dans l'ouvrage qu'elle lui a consacré et qui fut publié à l'Hexagone ${ }^{17}$. C'est Vigneault qui avait proposé à cette enseignante dans un établissement de jeunes filles, et qui faisait alors des études en lettres, de me prendre comme consultant pour cette recherche. J'ai d'ailleurs écrit la préface de cet ouvrage. Quelques années plus tard, quand l'éditeur Seghers décidera de publier un Vigneault dans sa célèbre collection «Chansons d'aujourd'hui », il m'avait encore suggéré comme auteur ${ }^{18}$. J'avais dû malheureusement décliner de faire cet ouvrage que j'aurais bien aimé faire, étant alors moi-même englouti dans [147] un travail qui allait m'accaparer encore assez longtemps.

Depuis lors, Gilles et moi n'allons plus nous revoir que de loin en loin, absorbés l'un et l'autre dans des carrières qui ne convergent plus guère. Mais je continue, comme simple spectateur, de suivre de loin cette carrière unique à tous égards et qui a littéralement explosé en France comme vous savez.

J.B. Toute votre production radiophonique pendant dix ans, au rythme d'une émission par semaine, puis les quelques articles que vous avez consacrés au phénomène de la chanson dans des revues d'éducation par exemple, vous les avez signés du nom d'Alain Sylvain. Pourquoi encore un pseudonyme?

17 En 1968.

18 Un Français, Lucien Rioux, en sera l'auteur en 1969. 
G.B. D'abord il n'y avait aucune intention de mystification comme dans le cas d'Isocrate, raconté plus haut. Ce nom était composé du prénom de mes deux fils, tout simplement. Je n'étais pas allé chercher loin. J'avais choisi un pseudonyme par pure discrétion. Cette activité pouvait paraître assez loin de ma carrière. J'évitais ainsi les questions indiscrètes qui m'auraient entraîné à raconter comment j'employais mes loisirs de fin de semaine. Je ne le niais pas quand on me demandait de confirmer mon lien de «parenté » avec Alain Sylvain. D'ailleurs aucune remarque désobligeante ne m'a été faite. D'autre part, dans l'intervalle, j'avais dû faire quelques apparitions à la radio et à la télévision, et même à un collège, sur la chanson comme phénomène social et comme instrument pédagogique. De tout cela je ne garde que le regret de n'avoir pu faire quelque chose de mieux achevé et de plus intégré, par exemple le Vigneault de chez Seghers et surtout une «petite sociologie de la chanson », comme un collègue parisien m'en avait fait la suggestion. Ce que je ferai de plus complet à cet égard c'est un assez long papier pour le Magazine Maclean en 1971, dans lequel je faisais la synthèse de l'évolution, par décennies, de la chanson québécoise avec ses quatre piliers chronologiques : la Bolduc, Leclerc, Vigneault et Charlebois.

\section{J.B. Vous reviendrez peut-être un jour à l'étude de la chanson ?}

G.B. Je ne le pense pas. Ce divertissement analytique, ou cette analyse divertissante, correspondait à une étape particulière de ma vie, de mes recherches et il faut le dire à une phase de l'épanouissement du phénomène, les années 1950 en France, les années 1960 au Québec. D'avoir été un pionnier - comme pour Isocrate - dans un genre ne fonde pas une obligation à devenir un spécialiste de ce genre. Mon intérêt majeur était de divertissement, de plaisir poétique dans la découverte mais aussi d'un brin de sociologie proprement dite en me faisant explorer un domaine presque inconnu de la sociologie des loisirs. En ces années 1960, l'emploi du temps libre allait devenir un thème d'analyse sociologique très important. Il y avait aussi l'évolution des canaux et media de communication : radio, disques, télévision, retour aux micro-audiences de la boite à chansons et à la salle de classe. Sur-commercialisation d'une part, mais aussi émergence des spontanéités 
communautaires pour de nouvelles formes de communion justement. Poètes et chansonniers ont affirmé fortement, bien qu'avec une marge d'ambiguïté (il est de l'essence de la poésie de rester toujours bellement ambiguë, si j'ose dire), l'espoir d'un Québec indépendant. En tout cas, avant d'autres voix, avant les voix de la politique. Cela vaudrait d'être étudié de près comme important chapitre de notre sociologie culturelle.

Mes scripts de présentation des chansons ne parlaient pas ce langage (que j'emploie ici à des fins de concision). Mais c'était le même fond de discours en langage bien autrement simple. J'aurai contribué à montrer sur les ondes de Radio-Canada que la chanson n'était pas un phénomène frivole, à la seule consommation des jeunes qui veulent continuer à rêver en s'assemblant. J'aurai tenté d'illustrer, avec pièces à l'appui, que ce n'est « pas un genre mineur » car, selon l'axiome complet «il n'y a que des artistes mineurs ». À l'époque de la Commission Rioux sur l'enseignement des arts au Québec, il se sera fait des études sociologiques selon les lignes indiquées plus haut. Plus [149] récemment, j'apprenais que l'Institut québécois de recherche sur la culture commandite aussi des travaux de même nature. Poésie ou musique «mineures » si l'on y tient absolument, mais avant tout populaires, c'est-à-dire qui trouvent preneurs. Et cela relève au moins de la sociologie des communications. Quant à moi qui pouvais rester très sélectif sur les thèmes, les auteurs et les séries que j'entreprenais, je trouvais beaucoup de plaisir à laisser s'émanciper un petit côté «fleur bleue », que nous portons tous, mais en le cachant, la maturité venue, sous des entreprises dites sérieuses de grandes personnes. Bref, engagé dans d'austères travaux de ma discipline, j'y trouvais un moyen d'hygiène mentale...

\section{J.B. Mais depuis dix, quinze ans, au moment où vous avez cessé cette ac- tivité, la chanson n'est plus ce qu'elle était.}

G.B. Dans l'ensemble, avec tout ce tapage d'amplification par électronique et par éclairages crus, je ne vous cache pas que je ne m'y sens pas très accordé. La faiblesse des textes - parfois ramenés aux grognements originels de notre espèce et la cacophonie amplifiée techniquement auraient plutôt l'art de me rebuter. Sans doute ai-je des goûts démodés, et je ne m'en excuse pas ! Il reste tout de même 
que, souvent mauvaises jusqu'à en être bêtes et irritantes, les chansons sont toujours des documents d'époque - et qu'à ce titre, elles vaudraient de rester objet d'investigation psycho-sociologique. Mais comme votre question m'incite à un contraste rapide, je ne voudrais pas vous laisser sous l'impression d'un rejet global. D'abord, il reste encore place pour la chanson intelligente (pas « intellectuelle »...) et poétique (pas celle qui dit : «Voyez comme je suis inspirée... »). Les grands de la chanson durent encore. D'autres, en plus faible nombre il est vrai, réussissent à percer et vont peut-être durer. Suffisamment en tout cas pour que l'honneur de la chanson reste à peu près sauf. Ce n'est pas d'aujourd'hui que datent les entreprises d'abêtissement des hommes.

[150]

\section{J.B. Mais nous n'avons pas parlé de votre dernière série, Mythes et mons-} tres sacrés du cinéma, à laquelle vous attachiez une importance particulière.

G.B. Cette série de 1966-67 allait clore ma collaboration de dix ans à RadioCanada. Le titre en disait clairement le contenu. Des débuts du cinéma (Chaplin, Mary Pickford, etc.) jusqu'à notre époque (Bogart, Gable, Monroe, Bardot, Gabin, Belmondo, etc.), j'ai étudié les grands noms de stars ou mythes, ou « montres sacrés » justement, selon l'expression consacrée au théâtre. Et cela pendant cinquante semaines. Aux très grands, je consacrais plus d'une émission. À part ceux que je viens de nommer, bien sûr Greta Garbo, Marlene Dietrich, Fairbanks et autres jusqu'à James Dean, des metteurs en scène comme Hitchcock, Wille, Korda, etc., et bien d'autres pour près d'une quarantaine de sujets. Je me souviendrai en particulier de l'extraordinaire halo énigmatique de Garbo, de la très grande lucidité de Dietrich sur son propre mythe. Coupons court sur un sujet inépuisable en vous confiant que, sans le dire alors, je pensais constamment aux rapports qu'on peut établir entre les mythologies cinématographiques et les mythologies politiques. J'ai à l'esprit plus que les analogies rapides de super-vedettariats. Il m'est arrivé de me demander si la structuration générale de la personnalité de telle star ne recouvrait pas assez exactement celle de la personnalité de tel homme d'État de premier plan... Et les types d'adulation qu'ils provoquaient n'étaient pas tellement différents de l'un à l'autre... 
Mais le parallèle le plus suggestif m'était suggéré par les grandes vedettes du music hall : elles, aussi, sont dans une extraordinaire situation de force populaire et de précarité psychologique. Comment une presque naine comme Piaf, tordue par les excès de vie et l'arthrite, peut-elle tenir pendant des mois une salle de deux, trois mille personnes, suspendues à ses lèvres et au moindre de ses gestes, qu'elle avait du reste avares ? C'est un phénomène inouï que celui du one man show, qui peut aussi être celui d'une femme - pensez aux concélébrations autour d'une Diane Dufresne! Je me souviens d'une confidence de [151] celui qui est peut-être le plus grand, parce que le plus parfait, du genre : Yves Montand. Il parlait du tempérament de dompteur qu'il faut pour affronter et maîtriser dans le noir quatre mille paires d'yeux qui ne cherchent qu'à vous " dévorer ». Tout comme l'orateur politique qui doit, lui aussi, «jouer »de la foule. Pensons aussi à l'effroyable solitude sous le faisceau de lumière, sans le support d'un texte à répliques ni la présence physique des autres comédiens sur scène comme au théâtre.

L'homme politique doit aussi franchir la rampe et l'assistance est rarement toute gagnée d'avance, ni gagnée à jamais. Mais c'est au moment des grandes décisions, celles qui importent en terme de destin, que l'homme politique se retrouve dans une solitude douloureuse et paradoxale pour lui qui a tellement de "partisans »! Pensez que les vedettes de la politique et du show business n'ont finalement de réalité humaine que par le degré de ferveur qu'une foule, anonyme et changeante dans ses caprices et exigences, qui est toujours battue par les entreprises organisées d'une publicité souvent rivale, veut bien leur donner. Le fait d'avoir tant voulu « cela » ne devrait pas nous empêcher de voir leur très grande précarité psychologique. N'exister soi-même de façon aussi décisive que par la «permission des autres ", peut-on imaginer plus forte aliénation sous le couvert d'une puissance qui peut être assez réelle mais qui est toujours transitoire ou éphémère ?

\section{J.B. À vous entendre discuter de choses aussi disparates que le music hall ou la politique, du vedettariat ou du destin, c'est à se demander s'il n'y a pas du moraliste en vous. En récuseriez-vous l'épithète ?}

G.B. Mon Dieu, non! C'est un beau mot. Ce serait autre chose si vous aviez parlé de «moralisateur » ou, plus correctement, je pense, «moraliseur ». Mora- 
liste, celui qui écrit sur les moeurs ou sur la morale des comportements publics, ça n'est pas déplaisant. Mais, je ne m'en formaliserais pas, je le prendrais plutôt pour un hommage. J'espère que, lorsque je vous parle ou [152] lorsque j'écris, je n'ai pas le style moralisateur, ni encore moins prédicateur. C'est un ton qu'ont parfois des intellectuels, dont des universitaires, quand ils descendent dans l'arène publique. Il me semble l'avoir signalé à l'entretien sur le journaliste, je ne parle pas à l'impératif : «Vous devriez faire ceci ou vous interdire cela... » ou « Voilà ce que vous allez faire puisque je vous le dis... ». C'est une attitude naïve et, comme contrairement à l'adage, le ridicule ne tue pas, on peut faire toute une carrière avec cette naïveté-là qui pourra passer pour un franc-parler ! Dans des pays où l'activité intellectuelle est un peu plus sophistiquée que chez nous, c'est-à-dire qu'elle n'est pas portée principalement par les média, cela ne tient pas longtemps.

\section{J.B. À quel mode parlez-vous ?}

G.B. Je pense bien parler principalement au conditionnel par «si », ou, lorsque je m'emballe un peu parfois, au futur : "Vous aurez les conséquences... ». Je parle au conditionnel lorsque j'argumente en posant que « si vous faites telle chose, ou si vous la faites de cette façon-là, il s'ensuivra telle autre chose qui contredit votre intention, même si vous ne vous en rendez pas compte maintenant ». Ce n'est pas une attitude moralisatrice. Le moralisateur, qui cherche plutôt à imposer ses propres règles, paraîtrait un moraliste au petit pied. Il ne suffit pas de dénoncer les travers et les faiblesses. Il faut mettre l'homme d'action devant les valeurs qu'il prétend honorer et qu'il n'a que trop tendance - à cause de son sacré « réalisme » à célébrer verbalement en rappelant, quand ça lui est utile, que «la politique est l'art du possible ». Il n'est pas possible de dégager une morale publique, à « face humaine » si j'ose dire, sans le rapport à l'efficience de la conduite des autres, soit aux conséquences : pas seulement aux conséquences pour nous citoyens, mais aussi aux conséquences de ceux qui tiennent ces conduites. J'aime assez cette pensée de Montesquieu : « Les hommes d'État sont remplis de bonne volonté, mais ils ne savent pas comment s'y prendre ». 


\section{J.B. Avez-vous l'impression que cette attitude que vous décrivez est bien perçue?}

G.B. Il est permis d'en douter. C'est, en tout cas, ma réponse à votre interrogation. Sauf quand le message est direct et qu'il n'y a pas lieu de décoder le sens de l'interpellation, rappelons ce que nous avons déjà dit sur les messages-bouteilles à la mer.. Et la marée en sens contraire peut les emporter avec la mer - avec tant d'autres bouteilles perdues. Mais si la marée est montante et que des vents imprévus font éclater la bouteille sur des récifs ou les rochers du rivage, il y a chance que le message soit lu. Bien sûr qu'il ne faut jamais y compter ! Mais la chance, toute petite soit-elle, doit être courue. Par-dessus tout, il y a les tiers qu'il ne faudrait tout de même pas oublier. Nous avons parlé comme s'il ne s'agissait que de deux interlocuteurs : celui qui émet un message et celui qui en serait le destinataire. Ici, il faudrait ouvrir un développement sur les influences diffuses ou potentielles, ou retardées, ou rétroactives, etc. Restons simples. Faisons court en soutenant seulement que, pour que la foire aux opinions soit assez garnie, il faut que les opinions se manifestent, se renouvellent. Moralité : il ne faut pas cesser d'envoyer des bouteilles à la mer...

J.B. Gardons la «bouteille », mais changeons de métaphore. N'est-ce pas un peu le point de vue de l'observateur de l'extérieur qui regarde les mouches s'agiter à l'intérieur de la bouteille ?

G.B.

En effet la métaphore est changée ! Vos mouches enfermées dans le bocal n'y jouissent d'aucune autre liberté que de bourdonner en tournant en rond... Il faudrait d'abord libérer les mouches pour qu'elles puissent mener leur existence de mouches! Il faut donc ouvrir le bocal. Et faire davantage : aider les mouches qui l'auraient désappris ou ne savent pas qu'elles le pourraient, à voler dans un espace plus large... 
J.B. Ces métaphores de bouteilles à mouches et à la mer vont me servir de transition pour aborder le styliste dans l'essayiste. Car il y a un «style Bergeron ", qui ne fait pas l'unanimité mais qu'on remarque. Il m'apparaît fait d'une tendance à l'inversion, de l'usage d'images frappantes de la vie de tous les jours, de l'invention de petites trouvailles syncopées. Un « écrivant »selon la distinction de Barthes, mais qui est aussi un écrivain dont la langue n'est jamais indifférente, ce qui paraît peut-être davantage en lisant vos livres.

G.B. Oui, je porte une attention spéciale à la langue de mes écrits. C'est un fait effectivement assez rare dans le domaine des sciences sociales et dans le journalisme. Mais il y a de notables exceptions de stylistes : Vadeboncoeur, Dumont, Lise Bissonnette, Jean Paré pour les premiers noms qui me viennent à l'esprit. Dans mon cas le produit n'est certes pas toujours heureux. Je ne fignole pas pour fignoler. Et les effets de style ne sont certes pas recherchés pour eux-mêmes. Ce seraient mes expressions ramassées qui, apparemment, causent de la difficulté à des lecteurs plus familiers avec l'expression plutôt molle des journaux qu'on parcourt toujours rapidement. L'idée dont on est habité, il s'agit de lui donner le support de la forme écrite. Elle peut sourdre de la vie réelle qui ne relève ni de la fiction ni de l'introspection. Il y a une contrainte particulière dans notre domaine puisqu'on n'est pas d'abord lu pour le style, pour la forme. Il y a aussi que je dois me dédouaner de ce que j'appellerais la langue théoricienne. Et je n'ai pas principalement à l'esprit le jargonnage de nos sciences sociales ou leur franglais.

Une partie importante de ce que j'ai écrit l'a été dans cette langue d'abstraction et de densité, qui doit viser un maximum de sens, devenir aussi bien signe que signification par le concept, se ramener à une série de propositions logiques interreliées en forme verbale. À l'autre pôle, il y a aussi que je dois m'alimenter par d'énormes quantités de papier journal dont la langue, aussi bien en anglais qu'en français, laisse à désirer. Je me suis rendu compte de l'influence insidieuse de ces deux déformations lorsqu'un religieux [155] enseignant, qui me suit depuis mes débuts, m'à fait observer que j'écrivais mieux quand j'étais plus jeune. Je me suis mis à faire davantage attention. 


\section{J.B. Comme langue universitaire, il y a pire que la vôtre, qui s'élève tout} de même à une certaine littérarité. La plupart de vos collègues écrivent en forme plain et sans personnalité. Il y a vraiment un littéraire refoulé chez vous.

G.B. Certes. Mais volontairement. Pendant mon adolescence, je me suis rendu compte que je cédais trop à l'imagination et à l'introspection qui sont sans doute des «défauts » nécessaires aux poètes et aux romanciers. À ce moment-là, je n'étais d'ailleurs pas sûr de mon talent, tellement il débordait dans l'abondance. Je lisais et écrivais trop, ne faisant guère que cela. Cette boulimie de lecture et cette logorrhée d'écriture étaient inégalement appréciées par mes professeurs. Par méfiance de moi-même, j'ai donné un solide coup de talon à ce trop fort penchant littéraire pour m'adonner, dans les dernières années du cours classique, à des écritures portant sur le monde réel de la vie sociale, qui est hors de l'Égo et le transcende d'emblée. Ça m'a libéré. Le « Je » courant de la vie quotidienne, il m'est apparu qu'il fallait le réduire à sa qualité de membre participant à l'espèce humaine et à la citoyenneté de tel pays. Ça vous aide au sens des proportions que de décider que vos états d'âme ne seront pas objet d'écriture, que votre Moi ne sera pas sujet de littérature !

\section{J.B. Vous avez donc combattu ce penchant consciemment et volontaire- ment ?}

G.B. D'autant plus facilement que je me rendais compte que j'étais aussi doué, en tout cas prolixe, pour les dissertations à sujets historique ou philosophique que pour les fabrications proprement littéraires de l'imaginaire. En 1942, alors que j'étais en rhétorique, [156] un éditorialiste alors renommé, Eugène L'Heureux, m'affirme que le journalisme offre un bel avenir, surtout dans l'analyse de la politique internationale. Tiens, me remonte encore à la mémoire qu'en ces années-là, j'avais fait une couple de « modèles » d'articles de commentaires d'actualité internationale que j'avais soumis à mon professeur de littérature. Après « correction », il ne m'avait pas dissuadé. En 1942, j'ai vingt ans, époque où l'on sort de l'adolescence et où il faut prendre partie sur des problèmes de l'homme, sans encore en 
être devenu un. D'ailleurs, y arrive-t-on jamais ? Le sympathique et fiable Gilles Archambault de Radio-Canada m'a interrogé là-dessus récemment avec quatre autres écrivains, deux d'une génération plus âgée, deux autres plus jeunes. Ce fut la première fois où je me serai quelque peu ouvert sur mes « vingt ans » - et sans doute la dernière. J'y étais un peu plus explicite sur le pourquoi j'ai opté de n'être pas un « littéraire » ou un écrivain au premier degré.

\section{J.B. Même si vous refusez la prétention du littérateur au premier degré,} je vous pose quand même la question classique : qu'est-ce qui précède chez vous : l'idée ou l'image?

G.B. Diable ! quoi répondre à cela qui est la question-type d'un littéraire à un littérateur? Vous me fournissez une autre occasion de regretter d'avoir accepté de répondre à toutes vos questions hors mon domaine réservé !

Essayons donc de voir comment cela se passe. D'abord, les rapports de l'idée à l'image vont dans les deux sens et varient d'une fois à l'autre. À un premier niveau des cogitations sans intention précise d'écriture, les deux s'agitent en complète anarchie et sans claire dissociation. Parfois, c'est l'idée qui pointe, d'abord, qui tend à prendre la forme d'une proposition, mais qui, aussitôt faite ou nouée, se décompose et va jusqu'à se contredire. Parfois, ça peut partir de ma vie sensorielle, par l'image comme vous dites. Mais alors on ne s'en rend compte que lorsque c'est déjà en marche. Quelle est l'image qui fait éclore ou qui [157] stimule l'idée jusque là larvaire ? N'est-ce pas plutôt une autre idée, parfois l'antithèse de la première, en attendant l'idée-synthèse qui peut être autant celle du point de départ que la proposition d'arrivée ? Surtout, tout cela est conditionné et porté par le nombre d'éléments - d'éléments d'information nouvelle sur les faits -, qu'on considère à tel moment ou qu'on retranche. Votre question force à un degré de conscience que n'a pas l'« écrivant » en écrivant justement. La feuille des griffonnages préalables à la rédaction, ce qu'on en biffe et ce qu'on y ajoute quand c'est parti, montrerait que ça ne se passe jamais de la même façon. C'est sans doute ce qui se passe pour tout le monde qui écrit. Mais je ne peux pas parler pour les autres. 
J.B. Mais il y a tout de même une recours plus fréquent à l'idée-imagée chez vous que chez les éditorialistes ou commentateurs : l'image du condominium pour le projet Souveraineté-Association, des frères siamois pour les grands partis, la bouteille à la mer de tout à l'heure...

G.B. Comment cela arrive, je ne le sais pas très bien. En philosophie 1ère année, on nous demandait de disserter sur l'axiome thomiste : Nil in intellectu quin prius fuerit in sensu. Les perceptions sensorielles aident-elles seulement ou sontelles la condition nécessaire d'expressions intellectuelles? Je ne saurais décrire cela avec précision. Tout peut servir de prétexte déclencheur sans rapport immédiat ou apparent à ce qui est déclenché : un arbre dans ma cour, la manchette d'un journal feuilleté, le panorama de la mer à Berthier où nous sommes en ce moment, la clé de contact dans ma voiture (qui me rappelle à tout coup l'époque des diligences), l'effet d'une bonne digestion propice à la rêverie ou la première pensée du matin, etc. N'importe quoi. Ou un objet trivial de la vie quotidienne : pendant que je vous parle, je tourne dans ma main le fourneau de ma pipe éteinte, ce qu'elle est la plupart du temps et qui fait qu'un fumeur de pipe fume surtout des allumettes ! D'ailleurs une des raisons pour lesquelles [158] je continue de fumer la pipe, c'est que j'aime le contact du bois qui est un matériau noble. Et surtout le bois de bruyère. Mais j'arrête ici pour ne pas déflorer le sujet d'un (court) traité de pipologie que j'écrirai un jour, oui ! Contentez-vous de savoir pour l'instant que ce ne sera pas un "défense » de la pipe, ni de la pollution, mais une «illustration » de l'art pipologique, car c'en est un ; et les pipologues sont plus rares que les fumeurs de pipe. Bon, arrêtons-là !

J.B. Il y a un plaisir évident des mots chez vous. Vous les secouez, vous précisez le vrai mot qui peut avoir valeur de concept ; vous rejetez ceux qui sont imprécis, surtout s'ils sont courants ; et parfois vous en inventez comme cette " pipologie " !

G.B. Nous avons touché la question des concepts à l'entretien sur le théoricien. J'aime aussi céder à des réminiscences et citations littéraires quand elles se mettent à remonter de la mémoire. Ainsi, quand j'ai à parler de la «marée », je 
l'appelle ainsi comme tout le monde. Mais dans mon monde intérieur, j'entends tout à coup « la mer toujours recommencée »... de Valéry. Depuis une couple de semaines, ces deux vers de Racine, où me paraît passer toute la tendresse du monde, ne me lâchent plus :

« Arianne, ma soeur, de quel amour blessé

Vous mourûtes au bord où vous fûtes laissée ? » Une autre fois, c'est l'absurde de la vie, et de la vie politique en particulier, qui m'accroche. Ainsi, par exemple, cette histoire de derrière le Rideau de fer que rapporte Jules Roy dans Les chercheurs de dieux qui vient de paraître :

- Quelle est la définition du capitalisme?

- L'exploitation de l'homme par l'homme.

- Et celle du communisme?

- Le contraire.

N'importe quoi peut activer ou déclencher un processus d'écriture.

Quand j'ai écrit mon Canada français en 1966, des extraits de nos poètes me sont apparus plus utiles que de fines analyses au fil du rasoir pour faire sentir à [159] nos amis français la remontée, après quelques siècles, d'un destin collectif qui aurait pu être autre : Miron : Moi, je gis muré dans ma boite crânienne.. ; Dumont: Moi qui suis de l'autre côté des mots...; Chamberland : Je ne veux rien dire que moi-même... ; et Vigneault, bien sûr: Mon pays ce n'est pas un pays, c'est l'hiver... et Je vous entends demain parler de LIBERTÉ. Simone et Jean Lacouture avaient choisi une photo de Leclerc, longtemps « le Canadien » à Paris, pour la page couverture. Quant à ce très beau poème de Jacques Brault, ils l'avaient sorti du contexte pour le mettre en exergue au volume. Tiens comme je l'ai en main, le voici : 


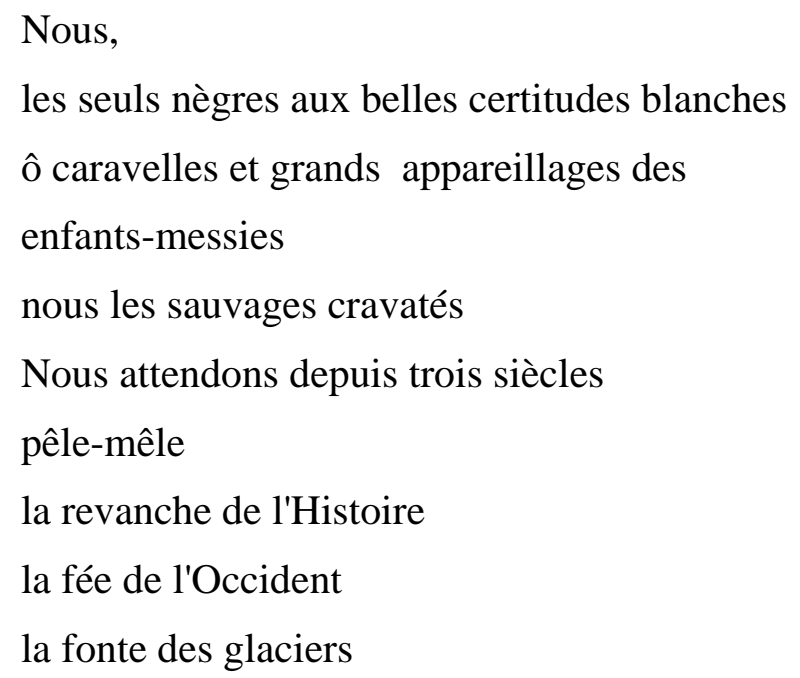

J.B. Nous sommes bien loin du style plain des analystes sérieux. Vous devez beaucoup vous refréner si vous vous défendez d'être poète vous-même. Mais le recours à une forme littéraire peut dénoter un manque de rigueur.

G.B. C'est fort possible. Notez d'abord que je n'ai jamais écrit de poésie. Remarquez qu'il est plus facile de faire dans le style plain comme vous dites. Il n'y a pas de rigueur expressive que celle qui se prétend « scientifique » ou « analytique ». Il y a aussi l'autre rigueur expressive, qui est la couleur de l'âme pour la vérité humaine à faire passer. La critique frémissante et passionnée de Mauriac dans ses feuilletons politiques leur conférait une qualité de rigueur particulière. Qui soutiendrait qu'écrire platement fait plus scientifique, plus rigoureux ? Rigueur de la pensée et « littérarité », comme vous dites, ne sont pas incompatibles, que [160] diable ! La condition minimale, c'est d'écrire correctement, toujours. Et ce n'est déjà pas si facile ! Écrire sans couleur, sans âme, sans personnalité, sans la toute petite pointe d'humour pour dédramatiser le sujet qui n'est pas toujours beau à raconter, n'est pas un indice de scientificité. Et soigner son expression n'est pas, non plus, indice de légèreté. 
J.B. L'auteur d'une thèse consacrée à votre oeuvre y note trois idées directrices, une triple volonté : d'idéalisme, de dynamisme et de totalité. Vous y reconnaissez-vous?

G.B. En première lecture, j'ai éprouvé un bizarre malaise, peut-être celui de me sentir à découvert... D'autant que cette thèse d'il y a une dizaine d'années était prématurée. Puis je l'ai relue en me disant: «Et,pourquoi pas ? Et si c'était exact ? » L' "idéalisme », ça me semblait assez difficile de l'éviter, comme tant d'intellectuels et de gens de notre espèce qui veulent changer des choses insatisfaisantes. De l'idéalisme, il en faut pour dévisager le pseudo-sens du réalisme si souvent invoqué par les justificateurs de n'importe quoi dont les injustices. Quant au « dynamisme », il tiendrait peut-être au fait que j'aurais plutôt tendance à dénoncer moins des maux ou des abus - quand j'interviens, c'est assez souvent déjà fait - que les inerties, les situations stagnantes et les inactions de gens qui pourraient agir. Cette idée se retrouverait aussi dans mes travaux théoriques : ma théorie de l'État est celle de son fonctionnement, de "comment ça marche » cette énorme machine et non pas tellement comment c'est "structuré » (selon une épithète dont on abuse dans le langage courant parce qu'elle fait savante, sérieuse). Ainsi dans mon étude de la guerre froide, j'ai détecté des cycles de tension et de détente qui semblent propulser les acteurs, en l'occurrence les deux Supergrands, U.S.A. et U.R.S.S., à agir de telle façon que, tenant compte de l'autre, ils se contrent mutuellement sans se paralyser complètement.

Mais l'idée de «totalité » je m'y suis encore davantage reconnu. C'est la vie des ensembles qui me [161] fascine. Ce point de vue holistique, ou gestaltiste comme on dit en psychologie, comporte un envers qu'il serait illusoire d'amenuiser : c'est celui de voir moins précis. Je travaille au niveau des ensembles en théorie ou dans des essais, quand ce n'est pas en journalisme lorsque des articles se présentent en petits essais concentrés : théorie de l'État, des relations internationales, toute l'époque d'après-guerre ; ou encore, à l'échelle d'ici : le destin politique global des Québécois, la qualité démocratique de notre politique, le système constitutionnel canadien. Je ne suis pas devenu un « spécialiste » de quoi que ce soit ni d'une discipline particulière. Quand je m'intéresse aux problèmes d'ici, je le fais moins en tant que «québécologue » - nous n'en manquons plus ! - mais en tant que citoyen, mais ça, aussi, c'est une totalité ! Cet instinct de saisie des totalités 
était certes assez fort pour avoir l'idée de construire des grands schémas d'interprétations théoriques. Je vous dis tout cela parce que vous me le demandez, mais en toute simplicité. Mais ça devient comme une "spécialité » lorsque des textes sortent. Et comme «il faut de tout pour faire un monde »... Ou pour évoquer un autre dicton, « ceux qui connaissent bien les arbres ou les bosquets ne sont pas nécessairement ceux qui connaissent la forêt ». Les uns et les autres doivent finir par se faire écologistes. Il en est de la nature socio-politique comme de la nature tout court, nous vivons au sein d'éco-systèmes.

J.B. En somme, vous plaidez la cause de ces esprits curieux et indisciplinés qui s'accommodent d'une connaissance limitée sur un grand nombre de sujets ! Après six ans d'études universitaires, quinze ans d'enseignement, de recherche et de préparation théorique sans publication, puis quinze ans de production intensive amorcée en 1965, vous voilà arrivé au projet des oeuvres de la maturité, comme vous aimez caractériser la période qui commence. On aurait l'impression que vous avez planifié votre vie.

G.B. Après coup, on dirait bien, en effet. Mais, ça n'a pas [162] été le cas. Je savais que j'écrirais une oeuvre politique, c'est tout. J'ai dit lors du troisième entretien comment je suis devenu théoricien, ou «théorisateur » que je préfère dire, sans trop m'en rendre compte, un peu à la façon d'un pensum que je m'imposais, un peu sur le tard du reste. Pour le reste, des circonstances extérieures ont pesé : le service des programmes d'enseignement en évolution, les problèmes fondamentaux de méthodologie de la discipline sur lesquels je butais, ou encore des faits accidentels comme les rencontres avec mon premier doyen Lévesque, avec André Laurendeau et même avec Gérald Godin ainsi que je le rappelais plus haut. Des choses semblables se produisent pour beaucoup de gens. Mais à ces circonstances extérieures ne dépendant pas de moi, je réagissais sans doute par des nécessités intérieures. Un peu comme celle qui m'a fait marcher dans le projet de ces entretiens alors qu'il reste tant de choses à faire.

\section{J.B. Et l'enseignement dans cette planification souple?}


G. B.

La priorité des priorités. C'est l'axe de tout le reste qui s'y greffe. À ma convenance ou pas, comme tout professeur consciencieux, il faut être prêt à donner son cours ou à le refaire s'il souffre de ce que j'appelle de l'arthrite. Comme j'enseigne de plus en plus sur l'objet même de mes recherches et que je publie là-dessus, l'enseignement aide et porte même l'ensemble. D'autant que se colleter à des étudiants toujours nouveaux oblige à rendre sa matière communicable et le plus possible intelligible. Je n'aime pas le mot « vulgariser », qui fait vulgaire justement. Apprendre n'est pas facile. Mais il y a retour utile et fécondant. Si les étudiants comprennent peu ou point, ce peut être qu'ils restent en deçà de l'effort nécessaire. Mais ce peut être aussi que ce que vous leur livrez n'est pas a point, ou que vos méthodes sont inefficaces. L'apprentissage va dans les deux sens. Et pour le professeur, il n'est jamais fini. C'est une profession qui garde jeune.

La carrière intellectuelle c'est toute une vie, contrairement à d'autres activités qui exigent une force [163] musculaire ou un fort influx nerveux comme le sport, les affaires, l'action en général, politique en particulier. Pour le travailleur intellectuel, l'âge de la retraite, si la santé est encore bonne, ce sont les années de maturation qui peuvent être les plus productives et qui permettent même de rattraper les projets ajournés ou refoulés.

\section{J.B. En votre for intérieur, caressez-vous l'ambition de laisser une « oeu- vre " derrière vous ?}

G.B. Si je m'étais concentré sur un seul produit, par exemple en théorie politique, cette oeuvre serait probablement terminée. De même pour les essais proprement dits, selon le titre de cet entretien, j'en aurais probablement un plus grand nombre à mon crédit. Mais le caractère d'unicité de l'œuvre ne doit pas s'entendre dans un sens trop strict : car, finalement, je n'aurai traité que de politique, que de l'État, toujours Lui ! Toujours j'aurai parlé de politique, même quand je faisais des scripts radiophoniques sur le phénomène de la chanson. Il n'en demeure pas moins 
que je déconcerte un peu les colleurs d'étiquettes. Je ne vais pas m'en plaindre : j'ai toujours eu une sainte horreur des étiquettes. Tant pis pour les conséquences. Il est maintenant trop tard. 
[164]

\section{DOCUMENT \\ L'homo quebecensis}

\section{L'homo quebecensis}

(Extrait de Le Canada français après deux siècles de patience.

Éditions du Seuil, Paris, 1967, p. 162.)

FICHE SIGNALÉTIQUE

DE L'« HOMO QUEBECENSIS »

NATIONALITÉ

double : $1^{\circ}$ britannique (la plus ancienne, qu'il n'a pas perdue en acquérant l'autre),

$2^{\circ}$ canadienne (aspirerait à n'en avoir qu'une, la québécoise).

LANGUE

$$
\begin{array}{ll}
\text { triple : } & 1^{\circ} \text { française (en principe), } \\
& 2^{\circ} \text { jouale (spontanément), } \\
& 3^{\circ} \text { anglaise (facultative mais indispensable). }
\end{array}
$$

FAMILLE

mère : la France

belle-mère : l'Angleterre,

cousins très lointains : à Jersey et à l'île Maurice.

\section{TUTELLE}

le clergé (conjointement avec l'Angleterre après l'abandon de la France).

\section{RÉSIDENCE}

locataire au second étage de l'Amérique du Nord sur le même palier que les « Canadians », occupant l'un des dix appartements, mais le plus grand.

\section{VIE SOCIALE}

isolement (aimerait pouvoir dire quelque chose à quelqu'un d'autre).

\section{GOÛTS INTELLECTUELS}

histoire (anciennement), poésie (un peu), roman (beaucoup), la télévision (passionnément, ce «divertissement des peuples qui s'ennuient ») ; semble imperméable à la philosophie et à la théologie.

\section{PSYCHOLOGIE}

idées fixes : maniaque de l'introspection collective ; dans ses cauchemars, s'imagine souvent en David sans fronde entre deux Goliath ; remords, par- 


\section{L'homo quebecensis}

(Extrait de Le Canada français après deux siècles de patience. Éditions du Seuil, Paris, 1967, p. 162.)

\section{FICHE SIGNALÉTIQUE} DE L'« HOMO QUEBECENSIS »

fois obsessif, de n'avoir jamais « essayé »de révolution. complexes : du « né pour un petit pain »; du «demi-succès et du demi-échec »; du " politique d'abord » (après avoir pris la politique à la rigolade depuis un siècle).

\section{PROJETS}

imprécis (mais forte tentation d'émancipation).

\section{CLASSEMENT}

- une catégorie à lui seul (trop différent des espèces voisines pour leur être assimilé).

- en eut deux, et tous deux hommes de guerre. Berthier, le nôtre, est : 
[165]

GÉRARD BERGERON.

de l'autre côté de l'action.

$6^{\mathrm{e}}$ entretien

Génération et environnement

«...l'idéal d'une philosophie

hédoniste du travail » 


\section{J.B. Estimez-vous que votre génération intellectuelle a été à la hauteur de sa responsabilité sociale?}

G.B. D'abord, on ne choisit pas d'être d'une génération. Personne ne peut prétendre parler en son nom. Je n'accepterais pas qu'on parle en mon nom sous prétexte que j'appartiendrais à une génération qui n'est après tout qu'un fait accidentel de naissance. Un analyste d'une autre génération serait mieux à même d'en faire une critique globale. Et puis, les différences de génération s'estompent et se confondent avec le temps. Parmi nos jeunes professeurs de la fin des années 40, certains ont déjà atteint l'âge de la retraite. Nous sommes donc les prochains sur la liste fatidique.

\section{J.B. Ce qui est l'occasion de rappeler qu'une génération intellectuelle,} c'est très court en notre milieu.

G.B. Bien plus court qu'une génération biologique. C'est non seulement un phénomène de jeune pays, mais de l'instauration de jeunes sciences dans un jeune pays. Ça prend, en effet, à peine cinq à six années pour faire basculer la condition d'étudiant en statut de professeur. Ceux à qui on enseigne, même si on n'a que quelques années de plus qu'eux, nous voient dans « l'autre génération ». Un jour, j'ai été interpellé par un étudiant: "Vous autres, les gens de votre génération, [168] vous... ». J'avais 32 ans : ça m'en a foutu un coup... !

Mais la notion de "génération » s'allonge avec le temps. Sans subtilité, on peut s'en remettre à des catégories chronologiques générales : les anciens, les précurseurs dont certains vivent encore et sont à l'âge de la retraite ; les aînés en pleine production, la cinquantaine dépassée ; la génération moyenne des quadragénaires; et enfin la génération des jeunes qui sont encore en deçà de leurs quarante ans. 
J.B. C'est cette génération des " aînés ", où vous êtes, que vise ma question.

G.B. Vous avez à l'idée ceux des intellectuels qui ont oeuvré dans et par les sciences sociales à partir de la base universitaire. Je crois que, dans l'ensemble, le bilan « générationnel » est largement positif. Pensez à ce que cette génération de l'après-guerre a fait avant 1960 : l'implantation massive, peut-être trop rapide et pas assez sélective, de ces sciences dans nos un universités ; la relance des grands journaux, le peuplement d'organismes comme Radio-Canada et l'O.N.F., la fondation et la réanimation de mouvements de base comme les syndicats, les coopératives, les associations professionnelles ; la présence dans les grandes fonctions publiques, la fédérale d'abord, puis la provinciale lorsqu'elle s'est ouverte après 1960. Cette génération a lancé et popularisé sur la place publique les idées qui allaient être au pouvoir à partir de 1960. C'était toute une présence au milieu 19. Ce que nous avons fait, plus ou moins bien, je le concède, le milieu québécois l'attendait puisque la suite allait montrer qu'il était prêt à le recevoir.

\section{J.B. Je restreins alors ma question à ceux de votre génération qui ont pas-} sé leur vie à l'université et dont c'était l'oeuvre majeure : par l'écrit, la parole, l'enseignement et surtout la recherche, bref de l'oeuvre intellectuelle à réaliser.

G.B. Là, c'est plus subtil. Dans cette espèce de [169] cosmogonie des sciences dites « humaines », je connais en particulier les sciences sociales et parmi cellesci, par métier, j'ai suivi d'assez près trois ou quatre d'entre elles : la sociologie, la science politique, le droit public, l'histoire politique récente et celles des idées, beaucoup moins la science économique, par exemple, qui est sans doute ma plus grande lacune. Vous parlez bien d'« oeuvre intellectuelle à réaliser » : ici, il faudrait pondérer. Quelques ouvrages ont affirmé une classe comparable à ce qui se

19 Gérard Bergeron a traité moins sommairement de cette période dans L'État du Québec en devenir (sous la direction de Gérard Bergeron et Réjean Pelletier, Montréal, Boréal Express, 1980). Voir le premier chapitre : « Les transformations socio-économiques entre 1945 et 1960 ». 
fait de valable dans des sociétés plus larges et mieux équipées que la nôtre. Mais, en seconde réflexion, l'énuméré serait plutôt court d'oeuvres véritablement marquantes.

\section{J.B. Donc, le bilan n'est pas tellement positif?}

G. B. Ici, il faut être aussi juste que franc. Presque tous les social scientists de notre génération se sont donnés une vocation que j'ai déjà qualifiée de « service social » (intellectuel) au milieu. Conséquence, ils n'auront guère analysé que l'un ou l'autre aspect de ce milieu social, le Canada français d'hier, le Québec d'aujourd'hui. Autre conséquence au second degré : de telles entreprises n'intéressent guère que le public d'ici, aussi restreint que circulaire. On se lit entre nous, sauf une poignée infime de spécialistes de l'étranger qui nous observent. Enfin, conséquence au troisième degré : les critères évaluatifs de ces entreprises sont à l'échelle du même milieu, c'est-à-dire ceux d'une petite société dont l'effervescence intellectuelle est très récente. Selon des critères un peu surélevés, très, très peu d'ouvrages passeraient la ligne d'exigence de la qualité, disons « internationale ». Mais je me sens un peu mal à l'aise pour vous livrer ces distinctions car, en dépit du soin que j'y mets, elles peuvent sembler présenter un relent de colonialisme intellectuel.

Un ouvrage, une entreprise intellectuelle qui se donne comme objet l'un ou l'autre aspect de la réalité québécoise ne sont pas, en tant que tels, pauvres en « scientificité ». Ce n'est absolument pas le point ». ce qui serait absurde ! Il s'agit de savoir si notre production [170] intellectuelle reste une simple histoire entre nous. Pour sortir du cercle restreint, il faudrait deux conditions : traiter de sujets plus universels et s'exposer à un niveau de critique plus large et exigeante. Les travaux portant sur les problèmes de méthode et d'épistémologie, sur les assises théoriques des sciences que nous pratiquons, par exemple, intéressent les hommes de sciences de partout et sont de leur nature exportables. Si, en outre, ils sont publiés par des éditeurs étrangers, ils tombent dans un circuit plus vaste où la critique risque d'être plus objective, « dénationalisée » si j'ose dire. Pour le dire d'une façon plus familière : on ne nous fait pas de cadeau ! On est tout nu, avec la seule validité d'un manuscrit. D'ouvrages qui ont subi cette confrontation, nous n'en 
comptons guère qu'une toute petite poignée, non plus que dans la génération d'après qui n'est pas plus audacieuse de ce point de vue.

\section{J.B. Vos ouvrages de théorie, par exemple.}

G.B. Mais ma propre production est pour moitié consacrée aux problèmes d'ici.

J.B. Justement vous savez de quoi vous parlez, puisque vous avez pratiqué les deux genres.

G.B. Je voulais seulement souligner qu'il y a deux standards possibles d'appréciation et que l'un est moins contestable que l'autre, celui de pairs mais à l'échelle internationale. Les gens des sciences de la nature, biologistes, chimistes, physiciens, etc.... entrent d'emblée dans un circuit plus vaste. Et certains, la plupart, l'élargissent encore en publiant en anglais ou dans des revues spécialisées de cette langue. Ce qui devient un problème d'une toute autre nature, hélas ! On n'entrera pas dans le dédale de cette question complexe où on retrouverait notre relatif isolement culturel et linguistique et la suprématie écrasante de l'anglais comme première langue scientifique internationale. [171] Soulignons tout de même, en fermant vite cette parenthèse, que le français est aussi une langue de communication scientifique, la deuxième, et qu'on peut en couvrir large par cette langue sur le plan international pour peu qu'on fasse montre de quelque originalité dans la créativité scientifique.

J.B. Vous êtes plutôt original pour vous être donné, dès le début de votre carrière, les relations internationales comme champ d'étude et d'analyse, ce qui vous a mené ensuite à la théorie générale de l'État...

G.B. ... sans laisser tomber des préoccupations sur les problèmes politiques d'ici, ainsi que nous en avons longuement discouru. 
J.B. Mais très peu de votre génération se sont attaqués à des travaux de pensée scientifique comme telle, immédiatement exportables. Cela aussi est un fait-témoin permettant de juger.

G.B. Il fallait partir, faire ce qui devait et pouvait d'abord se faire. J'ai l'air d'expliquer, peut-être même d'excuser, puisque vous avez posé votre question en terme de génération. Mais, vous dire que je ne suis pas un peu déçu de la performance générale, comme vous semblez l'être, les gens de la génération d'après, ce ne serait pas franc. Certains semblaient avoir un équipement intellectuel pour de plus grandes audaces et n'ont pas pris les risques des très longues et austères patiences. Je me souviens d'un mot, assez terrible, d'un grand intellectuel français qui déplorait chez nous un manque de ferveur dans la recherche scientifique et l'activité intellectuelle générale.

\section{J.B. Nos intellectuels sont sollicités par toutes sortes de tentations.}

G.B. Oui, certains n'y résistent guère. Il y a d'abord le fait [172] que les intellectuels d'ici percent trop tôt. Un de nos écrivains les plus doués me disait, il y a une quinzaine d'années : «Le malheur c'est qu'ici on plafonne très tôt ». C'était le cas pour ceux de notre génération dans les domaines que nous considérons. Étant les premiers à faire telle ou telle chose, on devenait facilement enclin à se considérer comme les meilleurs et à s'installer dans une situation facilement privilégiée.

Voici le schéma. Un ou deux ouvrages avaient attiré l'attention, du moins selon les canons d'ici. Mais le plus souvent, l'oeuvre elle-même reste toujours à venir, n'est pas venue. L'intellectuel arrivé est, très tôt, étiqueté, marqué. On lui demande de faire son petit numéro. On le balade d'un colloque à un studio de télévision à une présidence d'association, avec aller et retour. Il devient une petite vedette locale. S'il est le moindrement bon communicateur, ou s'il a quelque instinct d'entrepreneur, il supporte mai la séduction d'une réputation mondaine, via les media, les relations ou « contacts ». Il n'y a plus qu'à laisser aller et à donner 
quelques coups de pouce à l'occasion pour la reproduction du phénomène. On conçoit qu'un homme politique se trouve en concours de popularité permanent, qu'un athlète joue " pour la galerie », mais un intellectuel ne doit pas tomber dans les facilités du vedettariat.

\section{J.B. Certains ne se remettent pas de ces succès faciles.}

G.B. Comme vous dites. Il y a aussi une autre tentation, celle de la convoitise des commandites des corps publics, des commissions d'enquête, des associations professionnelles. Je n'ai rien contre le principe des expertises, ni même contre le fait qu'elles soient bien monnayées. Il vaut mieux faire ce type de choses que rien du tout, si on a besoin de ce type d'incitation pour travailler. On peut même y voir l'accomplissement d'un devoir civique. Un travail bien fait, commandité ou non, reste un apport à la vie de l'esprit, s'il tombe dans le domaine public. Ce n'est pas parce que je ne pratique pas ce type de travaux que je vais les dénoncer par je ne sais quel puritanisme de la science dite « pure »!

[173] Il y a aussi la question, complémentaire, des subventions de recherche. Il en faut, il n'y en aura jamais trop si l'on prend en compte l'envergure ou la technicité de certaines recherches qui ne peuvent être faites que collectivement. Ici encore, c'est une question de mesure. Je m'érige, par exemple, contre le fait de n'entendre guère parler de "recherche » qu'à partir de subventions, d'équipes, d'équipements techniques et, j'allais dire, de lointains échéanciers! Tant et tant de choses peuvent être faites préalablement et sans ces lourds outillages, surtout dans nos sciences sociales. Et puis, dans ces mêmes sciences, qui ne requièrent pas l'outillage technologique des laboratoires, il y a le corpus même de la pensée, objet et structure, qui doit finir par émerger des cogitations de l'esprit solitaire et besogneux. Vous avouerais-je que cela n'est pas ce qu'il y a de plus prisé par les administrateurs et évaluateurs universitaires ? Ils s'en remettent plus naturellement à des barèmes quantifiables, plus aisément lisibles, de « $\mathrm{X}$ » dizaines ou même centaines de milliers de dollars en subventions ou commandites, sans tellement se demander si la recherche aboutit vraiment, et, si elle n'avorte pas, si elle n'accouche pas d'une souris ! La nécessité d'un certain contrôle administratif des subven- 
tions et commandites par les autorités universitaires a entraîné subrepticement cette espèce d'inversion de la fin et des moyens.

\section{J.B. Effectivement, n'êtes-vous pas en train de me faire l'éloge de l'isole- ment forcé du chercheur d'autrefois?}

G.B. Je ne fais aucun éloge. Je dis seulement que la recherche solitaire ou artisanale, non commanditée et non subventionnée, doit garder toute sa place, et dans mon esprit cette place est prioritaire et non d'appoint. Elle est d'ailleurs à la portée de tous. Je dis encore que c'est une déviation assez grave que de mesurer l'importance d'oeuvres de l'esprit par des indices numériques. C'est plus grave pour la recherche en sciences sociales, qui n'a guère besoin la plupart du temps que de petits crédits de démarrage et [174] d'assistanat, que pour les sciences de la nature exigeant souvent des investissements matériels considérables. Nous touchons là un point qui n'est pas propre à ma génération de social scientists, ni au Québec. Après une trop longue période de disette et de parcimonie, on en est venu à surspécialiser et à surcommanditer la recherche, à ce point que la recherche dite «pure », non subventionnée et non orientée (vers des applications pratiques), que la recherche "gratuite ", en tous les sens du mot, en venait à paraître comme une originalité assez peu sérieuse ! Dans nos disciplines en particulier, Ronald C. Rowat de Carleton a lancé le cri d'alarme au Canada, il y a quelques années, par un article retentissant, The decline in free research in the social sciences ${ }^{20}$. Ce texte contient la structure d'argumentation que je ne puis évoquer qu'en passant.

\section{J.B. Vous semblez regretter ainsi l'implication dans le milieu au nom de} la recherche pure. On aurait pu s'attendre au reproche contraire !

G.B. Pour ce qui est de l'implication, il faut suivre la question jusqu'au bout. Dans le domaine de nos sciences humaines, cette implication est pour le moins

20 Canadian Journal of Political Science/Revue canadienne de science politique, IX, n 4, décembre 1976. 
ambiguë. Je ne vous déballerai pas toute la thèse de «l'intellectuel organique » chez Gramsci. Quels que soient le libéralisme et la largeur de pensée des commanditaires et subventionneurs, les chercheurs entrent dans un système où il n'est jamais complètement innocent de travailler sous de tels " patronages ». Je n'irai pas jusqu'à dire, comme on l'entend souvent, qu'on travaille plus ou moins consciemment à la consolidation du statu quo, dès lors que la plupart de ces commandes portent sur des projets de changements à opérer. C'est beaucoup plus subtil que ça. Et bilatéralement subtil... si l'on veut bien ne pas oublier que notre objet analytique est lui-même le pouvoir, en l'une ou l'autre de ses formes ou manifestations. Mon collègue, Léon Dion, dans un texte dont le titre même évoque cette ambiguïté, The political uses of political science ${ }^{21}$, en a particulièrement bien [175] traité. Les considérations que nous faisons en ce moment doivent s'entendre dans le contexte plus large d'une axiologie sociale et d'une déontologie professionnelle, qui fut celui des premiers entretiens. C'est quelque chose de bien autrement profond que ce que l'opinion appelle les classiques « conflits d'intérêt ». Je suis assez tenté d'aller, un de ces jours, jusqu'au bout de ma pensée sur les divers aspects de cette question que ni notre époque, ni notre milieu n'ont découverte. Car, plus que jamais se pose le problème de l'institutionnalisation de la recherche scientifique, de la science quoi !

\section{J.B. Je hausse la provocation d'un cran. La déception relative à l'endroit de votre génération vous englobe-t-elle ? Estimez-vous, en d'autre mots, avoir réussi votre carrière?}

G.B. Décidément, vous ne vous privez de rien comme question provocante ! D'abord, ma carrière n'est pas terminée. J'espère que mon avenir est encore devant moi. Et puis, il faudrait s'entendre sur le mot « réussir ».

Si c'est au fil des ans avoir obtenu, par ses travaux, un certain capital d'estime de la part de deux publics distincts, ce qui donne quelque confiance pour continuer, peut-être n'ai-je pas complètement échoue ? En tout cas, je n'ai pas perdu

21 Canadian Political Science/Bulletin de la Science politique au Canada, V, $\mathrm{n}^{\circ} 4$, mars 1976. En français, sous le titre de «Politique et science politique », Canadian Political Science, 22, 567. 
mon temps ! J'ai eu, comme tout le monde, de laborieuses hésitations, des va-etvient, des temps faibles à demi-perdus, mais je ne me suis jamais arrêté, comme le font les gens arrivés, qui se contentent de stimuler les intérêts du capital ! Comme vous le pensez bien, mon problème d'organisation principal est celui de la cohésion d'une carrière à plusieurs niveaux dont la compatibilité n'est pas évidente.

\section{J.B. Je voudrais en savoir davantage.}

G.B. Voilà bien l'aspect le Plus Périlleux de ces entretiens ! En vertu de cette convention initiale que je répondrais [176] à toutes vos questions, je ne vais certes pas me défiler. «M'exécuter », ça sonne bizarre dans un pareil contexte qui ne restera pas entre nous... D'abord, je ne jouerai pas l'indifférence ennuyée, ni la complaisance vaniteuse. Réussir partiellement, obtenir une certaine reconnaissance sociale, c'est nécessaire pour quiconque n'est pas installé dans la douillette médiocrité. Le degré de réussite dont il est question ne fonde pas une réputation si grande qu'il faudrait la prendre pour acquise, comme consacrée. N'exagérons rien! Par ailleurs, sembler ne pas porter d'attention à une certaine estime, que d'autres vous vouent, serait d'une modestie fausse et bien plus vaniteuse que la modestie affichée. Et qui, d'ailleurs, ne tromperait personne, surtout pas vous que je soupçonne d'un brin de perversité en soulevant des questions pareilles !

\section{J.B. Vous n'êtes donc pas insensible à ce que des gens expriment des opi- nions favorables à votre endroit, si je me permets d'insister.}

G.B. Insistance pour insistance, remarquez qu'on ne se tue pas à le dire ! C'est même la première fois que quelqu'un me pose une question aussi directe, passablement désarmante. On n'amène pas sur ce terrain des individus dont la carrière est terne, sans relief, à faible productivité. Pour aller au fond du fond, c'est moins du degré de réussite relative dont je suis fier que d'une espèce d'exigence morale à laquelle je m'astreins en me refusant de petites tricheries, certains trucs d'arrivisme dont le carriérisme intellectuel n'est pas exempt. D'ailleurs, comme c'est assez voyant à l'œil nu, cela finit par se retourner contre ceux qui cèdent à ces petites 
faiblesses. C'est d'ailleurs dommage, car c'est alors la valeur réelle qui en devient entamée. Non, il est certains modes de comportement en vue de la réussite que je n'emploie pas. En plus d'être un tantinet ridicule, l'auto-promotion ne fait qu'un temps et n'en impose qu'à un public sans défense, c'est-à-dire sans recours au sens critique, et qui suit les modes et les vagues à travers les réseaux qui consacrent ses chères vedettes.

\section{J.B. Arriver pas bien haut peut-être, mais tout seul, selon la formule de Cyrano?}

G.B. Cela pourrait se dire comme ça. Au total, sans avoir recouru à ces petites tricheries, peut-être ai-je relativement réussi, somme toute ?

\section{J.B. C'est par comparaison qu'on peut affirmer cela.}

G.B. Quelle comparaison ?

\section{J.B. D'une carrière à une autre.}

G.B. Alors non ! C'est même un point faible dans ma cuirasse. En quoi que ce soit, matière et manière, je serais bien incapable de me déterminer en rapport à ce que quelqu'un d'autre a fait ou prétend faire. Il m'arriverait même des messages indirects que je ferais mieux, pour mon propre avantage, d'être un petit peu plus conformiste. Ainsi, lorsque j'ai publié mes premiers ouvrages à Paris, un collègue, nullement mal disposé à mon endroit, insinuait que c'était parce que je ne trouvais pas que c'était assez important de publier en ce pays. La vérité était que ces deux ouvrages, fort différents du reste, devaient être publiés

par des maisons parisiennes pour trouver leur public naturel dans la francophonie internationale, y compris notre propre public. 


\section{J.B. Mais c'est peu courant que de s'imposer d'abord sur ce marché.}

G.B. Cela ne m'aura pas nui pour le reste, probablement. Mais rien n'est jamais acquis. Il y a aussi que je porte plus d'attention au motif de l'estime qu'au fait de l'estime lui-même. Un collègue parisien racontait que lui et une condisciple devinrent soudainement [178] dépressifs à la sortie de leur concours d'agrégation, qu'ils venaient de passer brillamment avec félicitations du jury et tout et tout... Pourquoi ? Ils estimaient que le jury faisait état de critères d'importance seconde et non pas de vraies raisons qui eussent fondé une note aussi élogieuse.

\section{J.B. C'est d'un certain raffinement.}

G.B. Il ne suffit pas seulement d'être en accord avec sa conscience, mais encore, pour ainsi dire, confortable dans sa propre psychologie.

\section{J.B. Vous n'êtes donc pas un homme de compétition ?}

G. B. Avec moi-même, certes ! Mais pas avec les autres, dans le groupe. C'est même assez lourd à porter quand on ne se connaît pas d'autre concurrent que soimême : on est voué à n'être jamais premier en quoi que ce soit, ni vainqueur de soi-même. J'ai toujours été plutôt mésadapté social de ce point de vue. Ni dans la famille, ni à l'école, ni à l'université, encore moins dans les quelques sports que j'ai pratiqués avec un talent médiocre et une concentration détestable pour les coéquipiers, je n'avais l'ambition d'être le premier, de me signaler par des performances, ni même de gagner tout simplement. Cette espèce de refus de courir dans le peloton peut avoir un revers pas tellement noble, qu'une psychologie sommaire décrirait peut-être comme la crainte inavouée de ne pas gagner. Je ne sais trop... En tout cas, ça m'est toujours resté. 
J.B. Vous êtes finalement plus le golfeur qui se bat contre lui-même que le joueur de tennis.

G.B. Je ne connais pas les règles du golf. Je me verrais [179] plutôt en cheval de labour, qui veut longtemps la même chose en prenant le temps de se retourner à chaque bout du champ.

\section{J.B. Ou un coureur de marathon ?}

G.B. Mais un marathonien solitaire qui n'oublierait tout de même pas qu'il a à remporter le sprint final.

\section{J.B. N'y aurait-il pas de l'anarchiste chez vous?}

G.B. Je parlerais plutôt d'un individualisme profond, qui fait que je ne me sens à l'aise qu'aux marges. Mais la marginalité, ce n'est pas toujours très bien porté. Il faut même savoir en payer le prix à l'occasion. Collégien, comme je n'étais pas trop « mauvaise tête », réformateur, etc., je jouissais d'assez de tolérance, même dans l'organisation très hiérarchisée des collèges classiques. Je ne semais pas la zizanie. À l'université de même, j'étais de la classe sans être vraiment dans la classe avec les autres. Bref, j'ai toujours été plutôt enclin à observer « le courant », sans tenir à être dedans.

J.B. Il aurait été préférable de laisser à la postérité une image un peu plus bouledogue de Gérard Bergeron : un homme de combativité, de ténacité, d'ambition...

G.B. Il faut même ajouter à ces « tares » une indécrottable paresse. 


\section{J.B. Un paresseux qui a écrit quinze ouvrages en seize ans ?}

G.B. Un paresseux qui travaille tout le temps..., c'est-à-dire à sa façon, qui n'est pas celle de tout le monde, qui [180] refait son énergie en travaillant pourvu que ce soit à son rythme. Sous cette boutade, vous comprendrez que ça me demanderait plus de volonté de faire autrement, de m'ajuster à des modèles courants pour l'emploi de mon temps en travail-loisirs, en travail-vacances. Au sujet des quinze ouvrages, il y en a deux qui sont collectifs, et il faudrait rappeler que j'ai publié le premier livre à quarante-trois ans. Je me suis préparé longtemps, si je me suis plutôt rattrapé depuis. Il y avait aussi sans doute de l'indétermination dans ce qui ne fut pas qu'une longue gestation. Les camisoles horaires du 9 à 5 et des cinq jours-semaine briseraient mes rythmes de travail. Heureusement que mon métier d'universitaire permet cette souplesse dans l'organisation matérielle de ma vie.

\section{J.B. Mais vous vous êtes trouvé à relever de grands défis intellectuels.}

G.B. Étant donné le point d'où je partais surtout. Mais çà m'attirait invinciblement dans les circonstances dites plus haut et que je ne pouvais prévoir.

\section{J. B. Encore votre instinct de marginalité.}

G.B. Si vous voulez. Ce n'est pas faute de ne pas avoir essayé de me corriger. Car j'en ai pris le parti sur le tard, pour le meilleur ou pour le pire. Enfant, étudiant, quand j'essayais de me couler dans les moules courants, je gaffais lourdement. Il en sera de même dans le métier, dans les associations. Je n'étais pas un « gars de gang ». Plus tard, je n'aurai été d'aucun « gang », ce qui déconcerte encore certaines personnes trouvant que ce n'est pas naturel et qu'il faut placer tout le monde dans des catégories étiquetées.

J.B. Les activités extérieures parallèles que vous avez menées depuis vingt-cinq ans, comme l'écriture journalistique, ne compensent-elles pas un 


\section{travail universitaire [181] à clientèle plutôt restreinte ? Ne révèlent-elles pas une certaine insatisfaction de votre métier principal ?}

G.B. Je ne crois pas. Si ma carrière s'est ramifiée en plusieurs activités, c'est en raison de la multiplicité de mes intérêts complémentaires. Je suis le premier à déplorer de n'avoir pu creuser chaque domaine avec autant d'intensité que si j'avais toujours pilonné le même secteur. Je ne sais trop : parfois, je me dis que c'est par nécessité, d'autres fois, que c'est par facilité que je suis devenu une espèce de spécialiste des idées générales, des mouvements d'ensemble. Mais c'est peut-être une des conditions du travail synthétique de l'élaboration théorique que d'être touche-à-tout ? Et avec le temps, au lieu de resserrer, ce qui eût été sans doute de bonne prudence, j'ai élargi plutôt. L'essayiste se trouve coincé entre le théorisateur et le journaliste-pigiste. Les trois peuvent se gêner. On ne peut tout faire en même temps. J'adopte le compromis de faire une chose après l'autre.

J.B. N'assiste-t-on pas un peu partout à une réhabilitation de la synthèse, de la théorie générale après les abus de la sur-spécialisation?

G.B. Mais, dans mon cas, ce fut par inclination naturelle depuis le début et non pas pour contribuer à cette « réhabilitation »comme vous dites. Il y a aussi l'avantage, fluctuant peut-être, à n'être pas l'homme d'une seule perspective, d'une seule spécialité. Mon oeuvre de théoricien, sur laquelle je cogite tout le temps, peut prendre du retard, mais n'est finalement pas neutralisée par mes explorations d'essayiste et mes incursions de journaliste. Les trois activités se portent l'une l'autre d'une certaine façon. C'est loin d'être évident à la lecture de mes travaux. Je me rassure toutefois par les croisements, les médiations et les interfécondations qui suggèrent des considérations diverses dont je n'aurais pas l'idée si je ne me livrais pas à cet éparpillement... disons consécutif.

\section{J.B. Vous êtes donc assez satisfait !}


G.B. « Satisfaction », je n'aime pas ce mot, qui me fait penser à la note scolaire, satisfecit en latin! La satisfaction évoque pour moi quelque chose d'assis, quelqu'un qui digère. On est assis quand on est satisfait. Ce n'est pas loin de l'inertie, tout au moins de l'activité végétative minimale. Ce serait bien étonnant qu'un jour, je devienne satisfait! Ça m'apparaît une espèce d'état second qui aurait fait taire la lucidité, la nécessaire autocritique. Ma carrière continue. La biologie tient. À l'âge de la retraite, si le second souffle devient trop court pour les longs labeurs de la recherche fondamentale, pourquoi pas la conversion finale au journalisme? Je finirais par où j'aurais pu commencer. Mais, en toutes hypothèses, je doute que je devienne jamais satisfait. Car, «satisfait », j'aurais le sentiment d'être mort même si je n'étais pas encore décédé.

J.B. Il y a quand même des signes extérieurs de satisfaction : prix, médailles, ordres honorifiques, etc. ?

G.B. Ce n'est pas rien si ce n'est pas tout. Il est de bon ton d'adopter une attitude désinvolte au sujet de ces "colifichets » auxquels il faut consentir comme rule of the game. Mais, le plus comique, c'est l'attitude presque de victimes que prennent d'heureux lauréats qui, parfois par eux-mêmes ou plus ordinairement par amis interposés au bon endroit, ont su donner les coups de pouce opportuns. Il faut d'ailleurs distinguer les prix qui se rapportent aux ouvrages, publiés entre tel intervalle de temps, tombant dans telle catégorie et qui sont soumis d'office à l'examen de jurys, des distinctions couronnant une carrière, l'ensemble d'une oeuvre, l'activité civique.

\section{J.B. Vous avez vous-même décroché des prix de la première catégorie...} [183]

G.B. Et sans savoir, en ces cas, que mes ouvrages étaient en lice pour ces sélections. Cela m'a fait plaisir. Je ne voudrais pas paraître au-dessus de ces vanités, 
bien que je serais bien incapable de m'activer le moindrement à ce propos. Quoi qu'on puisse afficher, personne n'est insensible à des récompenses ou à des honneurs.

\section{J.B. Pour la seconde catégorie?}

G.B. Toute ma vie s'étant déroulée aux confins de la vie universitaire, de l'institution littéraire, du monde de l'édition, des associations scientifiques, du journalisme et même de la politique, je puis dire, comme ce personnage de Racine, qu' « élevé dans le sérail, j'en connais tous les détours »! C'est assez gentiment comique tout ça ! Je ne vais pas vous improviser une petite sociologie des jurys et des prix...

\section{J.B. ... les petites intrigues, les combines, etc. ?}

G.B. Pas tellement. C'est plutôt le fait même de l'existence des circuits, des réseaux : de copinage, de familles idéologiques (un peu), de localité (Québec Montréal, beaucoup), de spécialité (les disciplines, encore plus), de génération ( « les anciens et les modernes »), etc. C'est très amusant, car tout finit par se savoir ou par se deviner ! - Cela mène parfois à des affrontements, des pactes de non-agression, des échanges de bons procédés ; mais c'est rare. Ça se déroule d'habitude en pleine normalité routinière selon une série de règles non écrites dont la première est évidemment la règle de l'impartialité totale des jurys et dont la deuxième est la règle de l'indépendance stricte de chaque juré.

\section{J.B. Cela mène aussi à des frustrations ?}

G. B. Oui, chez ceux qui comprennent mal qu'on les oublie. [184] Nous sommes une petite société où les réseaux d'influence ou de promotion sont très courts, bien qu'assez achalandés par ceux qu'on pourrait appeler des « congréganistes » qui sont, du reste, de toutes générations. J'ai l'air de me moquer, mais dès lors 
qu'on admet que ces « distributions de prix » aient une fonction sociale d'émulation, autant ne pas s'en remettre au hasard de la loterie, mais que s'en occupent ceux qui s'intéressent à ce genre de dispatching. Il y a même des «têtes à ça ", qui sont d'ailleurs assidues dans les structures d'autres establishments. Le plus prudent, c'est évidemment de se tenir complètement en dehors. Ainsi, on n'est jamais en position de sembler remettre quelque chose à quelqu'un.

J.B. Cette attitude est en pleine conformité au rôle très individualiste que vous avez toujours eu dans l'université ou dans le journalisme, ou en rapport à la politique dans son ensemble.

G.B. Comme je n'ai pas « la tête à ça », et que je suis passablement paresseux, pourquoi me forcerais-je ? D'ailleurs, les volontaires de tous âges ne manquent pas. Avec mon absence de manières, je ne risque pas de devenir un champion dans le genre.

\section{J.B. Cela risque de vous arriver, toutefois ?}

G.B.

Qui sait ? Comme on disait dans mon village, « chacun son tour dans le banc d'oeuvre ». Je jouirai alors, en pleine immodestie, de mon nouveau statut de « marguillier » de la « fabrique » intellectuelle ! Nous ne faisons que reproduire, en petit et en retard, ce qui se passe déjà, en bien plus grand et en plus rude, dans d'autres capitales intellectuelles. Mais changeons de sujet, si vous le voulez bien. Insister encore pourrait finir par présenter quelque chose de suspect !

J.B. Épuisons la veine de l'immodestie. Il y a du plaisir à [185] vous lire à cause du ton, du rythme, de l'emploi d'images. On a l'impression que vous vous faites plaisir en écrivant. 
G.B. Travail et plaisir s'entremêlent. Écrire c'est sortir de l'isolement. Autant le faire de bonne humeur, en n'arborant pas certaine angoisse existentielle qui vous étreint à certaines heures. Mes écrits courts sont des soupapes aux austères travaux à longue portée. Je suis heureux d'être un homme, un homme du Québec, de vivre à Québec qui est d'Europe et d'Amérique, un universitaire au Québec, un sociologue dont l'objet est l'analyse politique de notre société mais aussi de la grande communauté humaine sur la planète Terre. Et de pouvoir livrer le fruit de mes réflexions sur tout cela à différents niveaux de communication, et avec chance de rejoindre divers publics. D'un mot, je poursuivrais l'idéal d'une philosophie hédoniste du travail, bien que je sache que mon oeuvre ne me « satisfera »jamais, pour reprendre le vilain mot que vous avez employé.

\section{J.B. En vous écoutant, on a parfois l'impression que vous avez planifié vo- tre vie.}

G.B. Pas au sens d'un « étapisme » strict et impératif. Peut-être, oui, au sens large de la remise en question, à différentes phases, du projet global. Ce fut d'abord la décision de ne pas plonger dans le journalisme et de n'en faire qu'une carrière seconde, à côté de celle de l'enseignant-chercheur. Après coup, on voit plus clairement que cela s'est articulé en grandes étapes : six ans d'études universitaires, quinze ans de préparation que suivront quinze autres années d'intense production. Et maintenant à l'âge de la maturité, le bilan pour voir ce qui reste à faire de ce que je n'ai pas le droit de ne pas entreprendre. Seulement, cette fois-ci, il faut viser juste et prudent : en cas d'erreur, le délai de rattrapage est plus court.

C'est une des grâces de l'activité intellectuelle que l'âge de la retraite chronologique n'entraîne pas [186] la retraite professionnelle effective. On peut continuer en gardant l'illusion - nécessaire ? - que le meilleur est encore à venir. Tenez, j'ai vu, l'autre jour, un décalque sur une voiture de taxi à Montréal reproduisant la devise des Old timers au hockey. C'est une paraphrase de ce vers d'une vieille ballade de l'armée américaine, que le général MacArthur cita à la fin de son discours d'adieu au Congrès après avoir été limogé par Truman en 1951 : Old soldiers never die. They fade away. Les old timers du hockey - vous en êtes un, 
vous-même - ont donc comme devise : Hockey players never die. They become. Joli, n'est-ce pas ? Ainsi en est-il des intellectuels : ils deviennent en prenant de l'âge...

\section{J.B. Mais ne nourrissez-vous pas un désir encore plus secret ?}

G. B. Vous êtes impitoyable, si ! J'ai le désir, qui maintenant ne sera plus longtemps secret, de tout auteur que son oeuvre soit l'objet d'une critique d'ensemble, lucide et dure, qui en dirait la portée, les particularités, les faiblesses et les trous aussi. Qui la mettrait en perspective d'autres oeuvres du même genre et de l'époque. Qui jugerait peut-être qu'elle contient quelques pages, certaines propositions ou concepts, qui vaudraient de survivre. Bref, qui ferait ce qui se fait parfois pour des oeuvres littéraires. Je le dis en toute impudeur, car je suis bien conscient que nos entretiens qui s'achèvent fourniraient des armes qui pourraient se retourner contre moi ! Après cet aveu, je n'accepte plus de question.

\section{J.B. Mais le mot de la fin.}

G B. Rapport à l'hédonisme dont il était question tout à l'heure, je dirais : Finalement, il n'y a peut-être pas d'autre devoir que d'apprendre à être heureux et, autant que possible, sans trop rendre "malheureux » de son bonheur ceux qui nous entourent ! 
$[189$

GÉRARD BERGERON.

de l'autre côté de l'action.

\section{petite géographie personnelle}

\section{New York, Paris, et Berthier-sur-mer}

Hors les séances d'enregistrement de ces entretiens, Gérard Bergeron et moi avons parlé de diverses questions, entre autres de littérature et d'histoire, de vie quotidienne et d'environnement. À ce dernier propos, il revenait souvent sur la signification symbolique qu'avait pour lui le paysage de l'anse de Berthier-sur-Mer se déroulant devant nos yeux. Je lui suggérai de mettre par écrit ses émotions à ce sujet, texte qui, après le Prologue, aurait pu constituer le coup d'envoi à ces entretiens. De retour à Québec, il m'envoya un texte qui, par son importance même, débordait la fonction introductive à laquelle nous avions d'abord pensé. Tout le texte est une élaboration de cette pensée centrale : « Plus je suis accordé à ce point minuscule, plus je me sens du Monde. » Connaissant par ailleurs deux écrits de jeunesse, l'un portant sur New York où il avait passé l'été 1946 (voir le deuxième entretien) et l'autre (jusqu'à maintenant inédit) portant sur Paris et écrit en 1951 au retour de ses étude européenne, j'ai cru les reproduire ici, car comme disait l'auteur, « New York et Paris sont les prototypes urbains de notre américanité et de notre francité ». Cette trilogie me semble compléter, sous un angle pour le moins inopiné, le portrait de Gérard Bergeron et s'intégrer naturellement à ce livre-entretien. 
[191]

\title{
le corps et l'âme de New York
}

\author{
(août 1946)
}

$\underline{\text { Retour à la table des matières }}$

[193] «C'est un rêve de chambre de commerce en technicolor. » (Murray Schumach)

On connaît ordinairement deux New Yorks. Mais le Broadway et Wall Street n'épuisent pas la présence de New York. Ils en sont des signes, plus éclatants peut-être que d'autres qui contraignent à un temps d'arrêt : le voyageur pressé les voit davantage. Il rapporte chez lui un souvenir partiel et stéréotypé; dans sa chambre, il aurait pu faire le même "voyage » avec le Wall Street Journal et une bonne collection de cartes postales où se déroule la fugue multicolore du Broadway.

New York n'est pas un monde : elle n'est pas non plus la synthèse d'un monde. Elle se contente d'être le prototype de la ville moderne ; elle l'est avec audace, exagération, effronterie. Presque sans apparence d'emphase, des écrivains ont tôt fait de proclamer : "New York, résumé de l'Amérique ». Elle est plutôt un des moyens par quoi l'Amérique s'est affirmée. Si la force jeune, la prospérité et ce que j'appellerais l'optimisme nuancé d'une espèce d'arrogance de l'Amérique sont plus apparents ici que partout ailleurs, ils ne sont pas manifestes qu'à New York et que par New York. Un fruit mûri avant terme mais normal, non tombé par terre mais encore relié à l'arbre d'Amérique, telle m'apparaît New York. 
Elle est la ville-étendard d'un jeune pays, parti de peu et en retard sur ses éventuels concurrents, et qui les a tous évincés et dépassés. Elle est la Métropole, avec la majuscule, [194] la ville moderne qui a grandi trop vite, en hauteur, moitié pour épater, moitié par un besoin de concentration urbaine. Elle est la ville qui devait loger sur une île ; l'île n'est plus maintenant que le noyau de cette gigantesque cellule qui ignore le phénomène de scissiparité. Pourtant, en dépit de la démesure qui présida à ces entassements en cubes, terminés par des pylônes, de pierres grises et de briques rouges, elle n'écrase pas. Elle ne fait pas suffoquer. La conscience qu'elle nous donne de notre taille de microbe ne tue pas l'esprit qui est en nous. Son corps démesuré a une âme. On ne voit ordinairement que son corps ; il manque à l'image l'oblitération spirituelle. c'est la rançon d'un séjour furtif.

Cette âme, c'est son peuple. Comme sa ville, ce peuple est multiple, multicolore, omniprésent. Il l'aime passionnément ; il sait qu'il est citoyen d'une ville dont rêvent les autres peuples des autres villes. Il en est fier, sans chauvinisme ; il sait comme d'instinct que New York est unique, qu'elle méprise toute classification ou comparaison. Il blaguera volontiers à son sujet. Pour taquiner un ami new-yorkais, je lui parle de Times Square comme étant « le centre du monde ». « Well, ajouta-til avec sérieux, as far as New York City is concerned ». Ce peuple, ce n'est pas celui qui se balade sur le Broadway à 4 heures du matin, qui ne voyage qu'en taxicabs ou en limousines ; c'est celui qui, au bureau, à l'usine, au magasin, sur les quais, abat huit jours de travail par jour, qui bonde le subway aux heures d'affluence, qui le remplit à longueur de journée. Il n'est pas exactement le «petit peuple » des villes européennes.

S'il comprend beaucoup d'ouvriers manuels, il n'a pas de prolétaires, S'il compte plusieurs millionnaires, on ne les distingue guère dans la masse des gens de condition moyenne. Ici, la condition d'average people, d'average family est un titre de gloire. Passer inaperçu, anonyme au milieu de cette rivière de gens qui s'écoule lentement le long des trottoirs et s'embranche aux intersections des rues et des avenues, c'est assurer la quiétude de sa propre individualité. C'est communier à l'âme de la foule, sans amoindrissement de soi. Comme New York est la Métropole, son peuple est la Foule. Le million de personnes qui passe quotidiennement par Times Square n'est guère un phénomène unique. Le touriste qui loge à l'Aster ou au Waldort [195] sait-il seulement que plus de la moitié est venue en subway? S'il allait à Brooklyn, à Bronx ou même à Queens, il verrait le même 
flot humain, tout aussi bigarré quoiqu'un peu plus modeste d'intention et de portefeuille...

New York s'accommode de tous les paradoxes, On dirait même qu'elle s'en amuse. Elle est la Terre promise et aussi un premier champ d'expérience. Elle est le couronnement d'une carrière et aussi le sépulcre de perpétuels espoirs déçus. Elle est la ville où le succès est le plus facile, mais aussi le plus éphémère. Ici la chance n'est pas qu'un mot. C'est elle qui consacre les succès les plus éclatants, plus encore que le travail. Le plaisir est recherché avec frénésie, la beauté adulée, l'argent adoré ; tout cela crie fort, vit de publicité ; les adeptes de l'exhibitionnisme ne voient pas autre chose. L'exercice d'un humble labour quotidien, la recherche obstinée de la science et d'un idéal de vie supérieure, la pratique sincère d'un culte et l'adhésion à une foi sont des réalités moins palpables mais aussi certaines. C'est à la fois la ville où l'on s'amuse et travaille le plus, la ville du culte du veau d'or et du vrai Dieu, la ville où le tempo est le plus rapide, mais aussi où l'on flâne le plus.

À Wall Street, il faut ajouter Greenwich Village où des poètes et des peintres vivent en pleine gratuité. Aux somptueux et tapageurs clubs de nuit, aux grands cinémas du Broadway qui ne déroulent que des world premieres, il faut ajouter les 8000 églises de tous cultes bondées avant l'heure de l'office le dimanche, ses musées, ses conservatoires, ses instituts, ses grandes universités, Columbia, Fordham, New York, etc... C'est la capitale mondiale du music-hall, mais aussi celle de l'opéra, du cinéma, et du théâtre. C'est la ville des loisirs opulents, mais aussi la métropole des jeux de bazars à cinq sous. Partout est l'empreinte du « signe de piastre », mais c'est peut-être la ville qui compte le plus de parcs, de verdure, de squares, de pigeons qui s'accommodent très bien d'ailleurs de leur existence citadine. La discipline est sévère sans rigidité. $\mathrm{Si}$, dans tel quartier, les enfants n'ont pas de terrain de jeu, la police ferme une ou deux rues à la circulation pour en faire un terrain de jeu de balle. Le kid de New York comme le gavroche de Paris est inventif ; il sait prendre parti de toute situation. S'il a la fantaisie de jouer de la balle sur un mur où se trouve la défense : No ball playing, à l'aide d'une [196] craie, il changera la défense en appel : Now ball playing. C'est en un sens une ville très humaine. Son nom est synonyme de progrès, mais elle est aussi conservatrice. Elle a ses traditions, ses mythes, ses superstitions. C'est ici le règne de la 
spécialité : tout le monde est professionnel de quelque chose et spectateur des autres choses.

On y vit dans la rue, au bar, au théâtre, on y mange au restaurant, au comptoirlunch, parce que le home est trop petit. Cette vie métropolitaine, par son rythme rapide, exige une grande dépense nerveuse ; elle est âpre et doit être combative ; mais on y est autant en santé qu'ailleurs, on n'y meurt ni plus jeune, ni plus vieux. On n'y est pas plus malheureux et souvent on a l'impression d'être plus heureux. Pour un citadin qui passe la fin de semaine à la campagne, dix viennent du dehors se divertir à New York. Du trottoir, on peut observer et connaître l'âme de New York. Mais son corps gigantesque ne peut être aperçu que de haut, en avion ou de la tour d'observation de l'Empire State. De jour, le quartier des affaires apparaît comme un champ de monolithes dont on aurait aiguisé les sommets. De nuit, l'impression est celle d'un immense arbre de Noël dont on ne peut voir le tronc principal. L'île Manhattan se réduit aux dimensions d'un îlot; l'ensemble a une certaine symétrie sans monotonie, ni rigidité grâce au Broadway qui serpente et à la tache de verdure du Central Park.

New York est l'incarnation même de la métropole. C'est la vocation qu'elle poursuit sans cesse. Métropole d'un pays, puis de deux continents, elle est maintenant la capitale d'un monde en voie d'organisation pacifique. 
[197]

\title{
paris, capitale des métropoles
}

\author{
(mai 1951)
}

Retour à la table des matières

[199] Paris est une ville qui ne s'apprend pas. On sait Paris. On sait Paris comme on sait respirer, se servir de ses mains, dessiner ou jouer de la musique.

On n'apprend pas Paris ; il ne s'agit pas, non plus, de comprendre ; on ne découvre pas Paris. On revoit Paris. Ce n'est pas une question d'intelligence, de sympathie ou d'amitié ; c'est une question d'instinct. On prend contact avec Paris de façon médiate ou immédiate : voilà toute la différence.

Paris est un vaste élément au ras du sol et qui nous porte. On s'y trouve ou non à l'aise ; on n'y est jamais indifférent. Paris n'est pas un grand choc : il n'est guère davantage un faisceau de mille petits chocs. Paris est un monde de pierre, de béton, d'asphalte où les siècles, non pas à la seule façon d'un crépi de surface, ont incrusté une concentration d'humanité. Ne cherchons pas ailleurs ou au-delà de cette explication générale. Paris est faite pour l'homme : voilà son histoire, son originalité, sa grandeur. Parce que pour l'homme, Paris est aussi de l'homme.

N'avez-vous pas remarqué le pouvoir magique, le prestige de sorcellerie que contient ce petit mot de deux syllabes ? Paris ! Dès qu'on le prononce ou qu'on l'entend, ce mot produit, l'espace d'une seconde, l'effet d'une illumination. Ëtre parisien est un titre de gloire qui n'est déjà plus à l'échelle nationale. Être parisien, c'est connaître l'adéquation de sa condition humaine et de l'habitat urbain. On 
n'apprend pas Paris ; on n'apprend pas plus à être parisien. [200] On peut connaître Paris sans y avoir jamais mis les pieds ; on peut aussi n'être parisien que de naissance ou de domicile. L'origine ne fait rien à l'affaire. On n'est pas parisien de première, seconde ou troisième zone ; on ne devient pas parisien. C'est plus qu'un état d'âme ; c'est, comme je viens de le dire, un instinct qu'on ne saurait décourager, distraire ou susciter.

Le Canadien de langue française - ou le « Français canadien », comme l'on dit de nous en France - y vient à la façon du provincial qui veut en faire l'enthousiaste connaissance. Il s'établit entre lui et la ville je ne sais quelles affinités secrètes, qu'on ne saurait définir et qu'on gagne à ne vouloir pas trop analyser. Ces affinités secrètes ne sont pas d'ordre exclusivement esthétique et intellectuel, comme celles qui attirent à Paris l'étranger d'une autre culture. Elle n'opèrent pas en tant que Paris a quelque chose de permanent et d'universel qu'est apte à saisir tout esprit non médiocre. Elles jouent plutôt à la façon d'un charme dont la séduction est certaine, à la façon d'un pouvoir d'intimité presque familiale où le non-Français pénètre plus lentement et moins profondément que les «provinciaux » que nous sommes.

Il faut voir Paris comme un beau film se déroulant au ralenti et sans coupure. Le film de Paris n'est pas en technicolor ni en gros plans exagérés comme celui de New York. Il ne produit pas l'effet d'une grisaille un peu vaporeuse comme celui de Londres. Il a moins l'éblouissant soleil artificiel qu'une douce lumière sous le ciel délicat et pur de l'Ile-de-France. Le film de Paris est en noir et blanc. Entre ces deux extrêmes, s'étagent une infinité de teintes et de nuances. Le bon vieux noir et blanc est moins bavard et prétentieux que le technicolor, mais en dit autant, d'écrit peut-être mieux les choses. On a dit de Paris « Ville Lumière ». Est-il une ville où la lumière soit moins crue, plus discrète et plus chaude ?

En plus de s'établir en un ordre d'où la raideur est absente, les séquences du film de Paris ont encore un rythme rapide et l'enchaînement est parfois inattendu. L'unité fondamentale ne nous fait pas oublier que le film est divers et multiple comme sont vingt siècles d'histoire de France. Paris est un film bien construit ; Paris est un beau film.

[201] Le point initial du film, ses premières images qui font office de prologue nous montrent l'île de la Cité, immobilisée dans l'étau chaleureux des deux bras de 
la Seine. C'est le Paris surgi de l'eau et qui se souvient le plus de ses ascendances médiévales.

À l'origine, Paris était un petit bourg situé sur une île minuscule au milieu d'une belle rivière. Puis, sur les deux bords de cette rivière, le bourg a étendu les bras de ses points et a happé des morceaux de campagne qu'il a fait siens. Le petit bourg des lointains Parisii est devenu le chef-lieu d'un comté, puis d'une province ; et l'histoire, à partir de ce morceau originel, a commencé le jeu de patience de puzzle qui devait durer des siècles. Si bien que le petit bourg monté sur pilotis est devenu capitale de quelques provinces et, finalement, capitale d'un beau royaume. Paris, par les rois, a rassemblé autour de lui des terres. Et parce que le royaume était beau et grand, il a voulu une capitale digne de lui. Mais récemment - il y a de cela trois ou quatre siècles -, Paris devint la tête hypertrophiée d'un corps qui la supportait mal. La renommée internationale de Paris date de ce jour. Paris était unique ; et le monde la reconnaît encore.

On a souvent comparé Paris à une jolie femme. Jeune ou vieille ? On ne sait ; probablement à l'un de ces âges incertains où, par les raffinements d'une coquetterie plus subtile, la beauté sait se faire plus discrète et mystérieuse. Paris ne se fixe pas un âge parce que la ville sait l'artifice de tous les âges. Paris est comme cette femme élégante qui, sur un grand boulevard, glisse un pas indifférent d'où émane un charme opérant. Mais qu'est-ce, au juste, que le prestige de Paris ? Ce serait peut-être un peu de tout ceci, car l'analyse serait insuffisante comme l'énumération toujours incomplète : l'ordonnance des allées des Tuileries, le transept sud de Notre-Dame, les Champs Élysées, la Sorbonne, une coupe de champagne, le pont des Arts, Valéry en dialogue avec Pascal, le quai de la Mégisserie, les ombres de Villon et de Verlaine se reconnaissant aux abords de l'Hôtel de Cluny, l'Opéra, l'arche d'un pont enjambant la Seine, quelques pêcheurs à la ligne qui savent que le poisson ne viendra pas mais qui pensent quand même au goujon, la dernière mise en scène de Barrault dans un décor de Bérard, les petits « rats »qui font [202] des pointes, un vieux monsieur en barbiche et béret qui cherche une justification à ses préjugés dans un journal de parti, des gosses qui criaillent dans un parc, la flèche de la Sainte Chapelle et ce bout de dentelle, serti avec art et qui sort justement des mains des magiciens de l'Avenue Montaigne ou de la rue du Faubourg Saint-Honoré... 
Comme Rome, Paris ne s'est pas faite en un jour. Cette impression de la diversité dans le temps est une des plus grandes joies du visiteur à Paris ; mais Paris n'a pas fait son unique renommée d'une rétrospection dans le passé comme Rome, ou d'une projection dans l'avenir comme New York.

La Tour Saint Jacques ne jalouse pas la Tour Eiffel qu'elle interroge au loin par-delà la Seine ; la coupole byzantine du Sacré-Coeur de Montmartre et les tours gothiques de Notre-Dame sont l'indication d'une foi et d'une piété continues ; le Louvre et les Invalides ne se scandalisent pas des lignes ultra-modernes du Palais de Chaillot ou de celui de Tokyo. Paris est de tous les styles mais n'imite pas avec servilité. Si Paris ne crée pas tout à fait, c'est pour recréer ou pour faire mieux qu'ailleurs.

Paris sait s'adapter et pour cette ville, l'adaptation est un jeu, parce qu'elle possède l'art des communes mesures. Elle laisse à ses édifices publics et religieux, à ses places, à ses parcs, leur couleur d'époque. Elle peut, sans culte aveugle, les entretenir, mais la toilette neuve qu'elle leur fait n'est que rarement une restauration. Il y a, en effet, de l'iconoclaste tout autant que du fidèle chez celui qui restaure. Paris ne restaure pas mais conserve et entretient. Le Palais Bourbon, le Panthéon, la Madeleine ne sont pas composites, pas plus que la Sainte-Chapelle ou Saint-Germain l'Auxerrois.

Ces pierres sont enfumées par les années ou les siècles ; mais Paris ne les gratte pas jusqu'au sang * pour les faire paraître d'un ton qui ne fut jamais le leur, ni ne pratique la contre-façon d'un vêtement moderne. Cette sensation [203] d'authenticité temporelle est très forte partout à Paris. Cela fait une ville hétéroclite, diverse ; mais ses éléments ne le sont pas. Par l'intervalle de deux coups d'œil, on saute d'un, trois, dix siècles, mais toujours sans heurt et de façon franche. Paris ne dissimule pas son âge. Paris est du lundi le lundi et du samedi le samedi.

Tout cela fait plusieurs Paris. Et n'était-ce ce lien invisible mais flagrant entre les époques et les quartiers, on croirait que Paris est formé d'une foule de petits Paris, de gros villages juxtaposés. Mais le Paris moderne, le Paris d'aujourd'hui fait l'effet d'une ville préméditée. Multiple, Paris n'est pas anarchique. Quelques

* L'auteur écrit en 1951. C'était une dizaine d'années avant que le ministre de la culture, André Malraux, ne décrète l'opération nettoyage des édifices et monuments publics de Paris. 
Paris sont factices, à l'intention des touristes : leurs enseignes sont plaquées. Et cela hélas ! arrive dans certains Paris chargés d'histoire et d'intimité comme File Saint-Louis, le Marais et le Quartier Latin. Mais cela ne gêne pas outre mesure. Il faut y mettre le temps, et le vrai Paris, le Paris des plus ingénieuses subtilités consent toujours au hasard des rencontres.

Il y a le Paris mondain et artistique : les deux coïncident souvent et s'appuient l'un sur l'autre. Il y a le Paris qui passe par l'Opéra et les grands boulevards et celui qui s'installe à la rue Lepic ou qui s'attarde à la rue des Saints-Pères. Il y a le Paris des Champs Élysées et celui de la fontaine du Luxembourg. Il y a le Paris de l'« article de Paris », des antiquaires et des petits libraires, comme le Paris de périphérie avec déjà les longues cheminées fumantes. Il y a le Paris d'Auteuil et de Passy et aussi celui de la Porte d'Orléans et de Ménilmontant.

Tous ces pays coexistent, sans prendre garde les uns aux autres, en un état d'osmose vitale.

Paris a su se poser des limites en hauteur ${ }^{* *}$ et en étendue. Le Paris d'aujourd'hui n'apparaît pas le produit d'une improvisation séculaire. Ce grand corps a su échelonner sur des siècles le programme de sa croissance. Paris s'est interdit des excroissances prolifères : aucun monstrueux embonpoint, ni hypertrophie d'aucune sorte. Comme chaque année ajoute un anneau de plus aux cercles concentriques des troncs d'arbres, les murailles du siècle antérieur [204] craquaient devant une nouvelle poussée de sève ; mais, à un moment donné, Paris a dit : «C'est assez ! Je fixe mes limites ici ; au-delà, ce sera l'écorce de ma banlieue. »

Paris s'est donné plusieurs centres de gravité : rues et avenues ne semblent pas bâties au bénéfice presque exclusif d'un Times Square ou d'un Piccadilly Circus. Le Concorde ou l'Opéra ont des prétentions à cet égard ; mais de nombreuses autres places, Denfert-Rochereau, la Place d'Italie, la République, Saint-Augustin, opèrent une salutaire décentralisation. Les théâtres, par exemple, peuvent être plus nombreux sur cette série annelée de boulevards qui joint la Place Saint-Augustin à la République ; mais on en trouve aussi depuis Montmartre jusqu'à Montparnasse.

Quoique métropole financière et commerciale, Paris n'a pas fait sa vie d'un port, d'un entrepôt, d'une banque comme Londres. Paris ne s'est pas vu contraint,

** Paris, hélas ! s'est mise aussi « à la hauteur » ces dernières années. 
par besoin de concentration insulaire comme New York - à moins qu'il ne s'agisse d'un choix mercantile -, de gratter le ciel par cent pointes. Paris a pu s'étendre mais borner son étendue. Ses plus hauts édifices n'ont pas dix étages. Ici, ou là, des clochers, quelques tours et dômes surplombent les toits avoisinants, mais toujours avec retenue. Seule, la Tour Eiffel habite le brouillard nuageux. Mais encore, par pudeur ou souci de légèreté, elle s'est construite de longues tiges de fer et de mailles et non de pierres opaques : elle s'est ajourée. Elle ne gâte pas le paysage, ni ne constitue à elle seule un paysage. De partout on peut la voir; mais de nulle part, hormis de sa base, elle ne s'impose.

Du haut de la Tour, on voit le Paris diagramme, le Paris qui irradie en étoile, le Paris sinueux et infléchi, le Paris des lignes droites et des lignes courbes, les droites étant de l'homme de l'époque récente, les courbes, des premiers bâtisseurs et de la nature, et, au milieu de cet enchevêtrement de lignes, la plus grande et la plus large de toutes, et aussi la plus régulièrement infléchie, le grand boulevard de la Seine, où glisse le lent trafic de ses longs chalands que Baudelaire aimait tant.

Paris, à toutes les époques, est apparue comme une capitale mondiale. Depuis le Moyen Âge, elle a toujours su [205] être de son temps, «à la page », parfois avec un léger recul vite rattrapé, le plus souvent avec une remarquable avance.

Florence est peut-être plus belle, Venise, plus pittoresque, Washington plus fleurie ; mais Paris n'imite pas. Elle se refuse à tout compromis, à l'encontre de Rio de Janeiro, qui s'essaie à opérer la vaine synthèse de Paris et de New York. San Francisco, Chicago, Montréal sont, sans qu'ils y consentent volontiers, des sous-produits de New York. Parce que Paris est Paris, Lyon, Bordeaux, Bruxelles ou Genève en reçoivent quelque influence, mais se refusent au décalque, La renommée de Paris est tyrannique, et cela, non seulement à l'égard des autres villes, mais envers ses provinces dont elle draine à son unique profit le sang le plus riche, sans même dire "merci »! À tel point qu'on peut se demander si la France n'existe pas pour Paris, plutôt que la capitale pour les provinces. On a même pu dire : « Paris et le désert français ». Parce que c'est Paris, les provinciaux consentent à tous les assujettissements.

Mais Paris n'est pas une Babylone. C'est plutôt une ville aux véritables dimensions humaines, peut-être la seule qui permette à ce degré de perfection l'intimité réelle et l'anonymat absolu. Nulle part, on ne s'y sent noyé comme à Londres ou 
un peu suffoqué comme à New York. La vie y est trépidante, mais sans fièvre, c'est-à-dire en pleine conscience. C'est un tourbillon, un mouvement perpétuel, en comparaison de New York qui est une cohue ou de Londres qui est un régiment bien dressé. Tandis que New York étonne par son côté nietzschéen, ville bâtie pour une super-humanité, tandis que Londres, incitant à la fois au sensualisme et au libéralisme, présenterait un caractère lockien, Paris, au contraire, a un aspect nettement cartésien, c'est-à-dire que la ville est faite de clarté et d'équilibre, d'évidences premières et de nuances subtiles. 


\section{à Berthier-sur-Mer où commence « ... la mer toujours recommencée »}

(août 1981)

$\underline{\text { Retour à la table des matières }}$

[209] Ce fut sur un panneau de signalisation routière que j'eus mon premier contact avec Berthier-sur-Mer. Cette appellation se détachait en ligne de beauté rehaussant encore l'élégance naturelle de la surface verte à bordure blanche. Mais, en réflexion seconde, cette toponymie paraissait un tantinet inflationnaire : ...surMer. Déjà la mer sur cette Côte-du-Sud en deçà de Montmagny, à 50 kilomètres à peine en aval de Lévis-Québec ?

Au-delà du détroit de Belle-Isle, l'Atlantique, c'est sans conteste la «mer océane » des premiers navigateurs et des anciens géographes. En deçà c'est le golfe qui est une demi-mer intérieure, comme les lacs Saint-Louis et Saint-Pierre, formant des espèces d'hernies du fleuves, ce sont des demi-lacs. Mais la Mer, ou commence-t-elle vraiment?

À Pointe-au-Père, où les pilotes fluviaux rendaient le gouvernail aux capitaines de haute mer précisément ? Ou à la hauteur de la Rivière-Ouelle, jusqu'où remonte l'eau salée ? Ou encore, plus haut, à l'Islet, à quelques kilomètres en aval de Montmagny, et qui s'était proclamé «sur-Mer » comme pour préparer à cette patrie du capitaine Bernier le musée maritime dont le village allait devenir si fier ? 
Mais, renchérissant, voilà que Berthier, à quelque vingt kilomètres en amont, s'est aussi récemment rebaptisée Berthier-sur-Mer.

Cette désignation a "plus de gueule » que l'ancien nom de Berthier-en-Bas pour se distinguer de Berthier-en-Haut qui porte maintenant l'appellation banalisée de Berthierville, [210] ville située en face de Sorel et de ses îles. Enfant, je ne pouvais comprendre pourquoi Berthier-en-Bas pouvait être en haut de la carte, ni pourquoi c'était le contraire pour Berthier-en-Haut ! Mais lorsque nous chantions «Sur la route de Berthier », nous ne nous interrogions pas sur quel Berthier pouvait être au bout de la route, celui d'en haut ou d'en bas, à moins que ce ne fut quelque bourg imaginaire de France ou de Navarre. L'entraînante chanson suffisait avec ses histoires cocasses de cantonnier et de grosse dame, de tas de cailloux et de carrosse doré. D'ailleurs, pourquoi ne s'accommoderait-on pas pour la rime de quelque géographie d'invention?

Mais « Berthier » est aussi personnages historiques, car il y en eut deux, et tous deux hommes de guerre. Berthier, le nôtre, est Alexandre, capitaine du Régiment de Carignan dont les valeureux services lui vaudront l'octroi de la seigneurie de Bellechasse. À ne pas confondre avec son quasi-homonyme d'un siècle plus tard, Louis Alexandre Berthier, glorieux maréchal de France, qui fut un temps chef d'état-major de Napoléon. Comme la fortune changeait de camp, ce dernier allait se rallier à Louis XVIII lors de la première Restauration de 1814, mais cette adhésion mal calculée ne lui sera guère bénéfique. Il mourra pendant les CentJours, à l'étranger et dans des circonstances restées obscures.

Le Berthier du Canada s'était donc illustré un siècle plus tôt, au sein du célèbre régiment qui avait imposé la loi de la plus grande force aux Iroquois de plus en plus menaçants. Natif de Bergerac en Gascogne, Alexandre Berthier était calviniste. Il allait se convertir au catholicisme en 1672 et épouser, à Québec, une jeune fille de famille, Marie Le Gardeur de Tilly : «Un mariage - comme Paris aurait dit Henri IV - vaut bien une messe ! » Devenu commandant du fort de Sorel, Alexandre Berthier s'implantera plutôt du côté du Richelieu que des territoires giboyeux de Bellechasse, les bien nommés. À partir d'une concession obtenue en face de Sorel (territoire actuel de Berthierville et de Saint-Cuthbert), il allait s'y tailler une seconde seigneurie, celle dite de Villemure, où il résidera jusqu'à sa mort. Il avait auparavant cédé sa seigneurie de Bellechasse à l'un de ses fils. 
Voilà pour l'origine du Berthier des Montréalais et du [211] Berthier des Québécois, de celui d' « en haut » et de celui d' « en bas ». Alors que Berthierville est maintenant une petite métropole locale sur la rive nord, à l'entrée du lac SaintPierre, Berthier-sur-Mer n'est encore qu'un petit village, dépassant à peine le millier d'âmes qui, de la rive sud, regarde vers Sainte-Anne de Beaupré et SaintJoachim. Car la vue est toute dégagée sur l'autre rive, à cette hauteur où l'Île d'Orléans n'engorge plus le Fleuve qui s'élargit en estuaire et commence à devenir, censément, la Mer.

Sur la route de Berthier-sur-Mer, comme sur toutes les routes, y passent sans doute des " grosses dames », mais on n'y voit guère de « tas de cailloux » ni encore moins de " carrosse doré »; mais on y rencontre des caravanes de machineries lourdes puant l'asphalte chaud, et que répandent des gaillards au torse nu sous le soleil, ces « cantonniers » qui continuent à faire leur « fichu métier ».

La route est d'ailleurs double ; la moderne autoroute 20 et la 132, cette prolongation du Chemin du Roy vers la Gaspésie. Il est même conseillé den faire un bel itinéraire de ceinture : à l'aller, par la 20, pour arriver plus vite à Berthier à travers les verts vallonnements de Bellechasse, qui a taillé à vif dans des érablières devenues improductives mais restées belles et qui donne des aperçus imprenables des beaux villages du versant sud de l'Ile d'Orléans; au retour, par la 132, à recommander en fin de journée pour la beauté des couchers de soleil sur l'Ile et le fleuve, qui permet de longer à faible vitesse les villages au charme discret jusqu'à la somnolence (Saint-Vallier, Saint-Michel et Beaumont), tout en admirant une série de vieilles maisons, bien entretenues, dont peu ont eu besoin d'une restauration complète, ce qui accuse toujours le crime de désastreux abandons. Même la 20 se fait belle et diverse dès Lévis, alors qu'elle est si plate en tous les sens entre Québec et Beloeil ! La 132 honore en partant les promesses de la route du Bas du Fleuve et y ajoute l'intérêt d'un petit festival routier de maisons du temps où, sans le savoir, on en faisait des oeuvres d'art. Le plaisir de Berthier-sur-Mer commence dès le fait de s'y rendre à vitesse d'autoroute et se prolonge par la flânerie du chemin du retour en proximité visuelle du bras sud du Fleuve, celui de la grande navigation et du versant sud, le plus beau, de 1'lle d'Orléans.

[212] Berthier, anciennement en-bas, s'est donc mis à se donner des « airs de grand monde » : sur-Mer, ou by-the-Sea comme on dit au Nouveau-Brunswick. Pourquoi n'en aurait-il pas le droit autant que Carleton à mille kilomètres au bout 
de la Gaspésie, ou que l'Islet tout juste passé Montmagny ? Pourtant, le village n'avait eu qu'un destin aux ambitions forcément modestes : celui d'un rang unique, longeant Fleuve ou Mer. Saint-François, c'est déjà son arrière-pays occupant les deuxième et troisième rangs. Berthier semble, et est resté, un village comme tant d'autres, qui n'a pas de titre particulier à la notoriété. Son église, reconstruite il y a un peu plus d'un siècle, serait charmante si un banal commerce d'alimentation et un infâme entrepôt de tôle n'en brisaient pas la perspective de la façade. On y déplore encore deux ruines : le quai abandonné qui s'écroule et, tout à côté, le presque squelette d'un manoir, ancienne demeure seigneuriale dont la silhouette n'a pas perdu toute allure malgré les seules attentions assidues des vandales. Une fois de plus, peut-être trop tard...

Heureusement qu'il y a "l'Anse » ${ }^{*}$ comme disent villageois et estivants, qui est la grande fenêtre latérale sur la mer. Tournant le dos au village et à la triple « horreur » déjà dite, l'anse et ses maisons d'été sont tournées vers le large, en une diagonale jusqu'à un point d'horizon qu'on pourrait fixer aux environs de Tadoussac. La voilà, enfin, la Mer à perte de vue entre les Îles de l'archipel de Montmagny s'éparpillant à une douzaine de kilomètres. À droite près du rivage, c'est l'Île aux Grues, habitée et cultivée, reliée à la rive sud par bateau-passeur et aviontaxi ; à l'autre extrémité, c'est la Grosse Île, dite aussi de la Quarantaine. Les immigrants naguère devaient y séjourner ; puis on y soumettra les animaux de race étrangère à ces précautions sanitaires. Entre ces deux grandes îles, à travers récifs, bancs, îslets ou îlots, et îles par dizaines on ne sait trop, passe le chenal de la grande navigation. Cette région devient terrain de chasse d'oiseaux sauvages en saison.

La plus grande beauté de l'anse, c'est son fond du décor de l'autre rive : non pas Saint-Joachim qu'on ne voit guère même par temps clair, mais le massif de Charlevoix qui [213] s'impose au niveau de la mer et en force avec ses deux hauts lieux que sont le Mont Sainte-Anne, paradis des skieurs et station renommée de compétition internationale, et, à quelques kilomètres, le Cap Tourmente, nom redoutable pour une belle forme arrondie, qui semblerait n'avoir d'autre rôle que

* Je mets l'Anse entre guillemets parce que l'Anse de Berthier, sur les cartes géographiques officielles, est une surfa-ce d'eau, bien plus vaste, un peu en amont entre Saint-Vallier et Berthier. C'est par « la rue de l'Anse », justement, qu'on accède à « l'Anse ». 
de faire pendant au Mont Sainte-Anne. Permettant d'embrasser d'un même regard les deux plus beaux sommets des Laurentides de la région québécoise, la plage de l'anse est une espèce de banc privilégié pour contempler de loin l'entrée du beau « pays de Charlevoix ».

vL'anse qui, à travers les îles, s'ouvre sur la ligne d'horizon de la mer, peut aussi presque se refermer en lac par je ne sais quel sortilège d'optique où montagnes lointaines, rives et îlets prochains, marées incessantes, brouillards légers et mirages de soleil se livrent à une espèce de double jeu d'ouverture-fermeture. Tantôt c'est la mer qui mugit par vent de nord-est avec ses «moutons blancs » venant racler le fond de la plage de tuf rouge ; tantôt c'est un beau lac calme, bien en deçà du chenal et de ses courants traîtres, lieu idéal pour les sports nautiques dont la rage récente de la planche à voile à vocation plutôt chavirante...

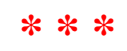

Pour moi, c'est toujours, comme cela fut au moment de cette découverte inopinée à une demi-heure d'autoroute des ponts de Québec et Pierre-Laporte :

«... la mer toujours recommencée ».

C'est pour l'évocation espace/temps du vers de Valéry que j'ai fait de l'anse de Berthier-sur-Mer une seconde patrie, celle que j'ai moins choisie que reconnue d'emblée lors d'une balade fortuite d'automobile il y a quelques années.

Je ne vais pas vous chanter l'intimisme des petites patries, du Small is beautiful, des paysages accordés aux états d'âme. Non plus que ce tardif impératif d'enracinements volontaires, complétant des racines naturelles : de Charny, village natal avec ses merveilleuses chutes presque à l'embouchure de la Chaudière, de Lévis où j'ai fait mes études classiques, de Québec où j'habite et pratique depuis toujours mon métier d'universitaire. Mais la belle [214] dualité d'ouverturefermeture de ce paysage unique fait de Berthier-sur-Mer un lieu où mes pensées, extensibles ou limitées, se meuvent à l'aise en toutes espèces de jongleries aussi débridées que spontanées.

Oui, on aime la mer parce que «toujours recommencée »... On aime la voir plutôt que la regarder, puisqu'elle s'impose du fait qu'elle est là, toujours la même 
et toujours autre. On aime différemment le lac, la rivière. Le lac qu'on embrasse d'un seul regard, m'est toujours apparu narcissique. Plus généreuse, la rivière est aussi paresseuse par ses méandres et affairiste par ses rapides. La mer, c'est autre chose. Ce n'est pas qu'une question d'étendue ni de perspective. La mer, toutes les mers sont en intimité tellurique et en liaison cosmique par la perpétuelle redondance des marées. La mer est plus ouverture que sortie. Elle s'ouvre sur quelque chose d'autre et de plus que la nappe d'eau que le regard permet de balayer.

Surtout lorsque des montagnes sont, si j'ose dire, à la hauteur des circonstances et forment les arrière-plans du paysage marin : à gauche, sur l'autre rive, le beau duo du mont Sainte-Anne avec ses pistes de ski visibles en toute saison et le Cap Tourmente qui salue la mer naissante en baignant dans l'eau ; mais aussi à droite, sur la même rive au-delà de la Pocatière, la ligne bleue et plus ténue des Appalaches. Entre montagnes aux deux rives, les îles de l'archipel de Montmagny émiettent la ligne d'horizon.

Moi qui me défends toujours de tout lyrisme, je ne fais qu'essayer de fixer des perspectives, de déterminer des distances. Mais j'avoue une prédilection pour ces lieux et aussi ces temps, qui sont comme de passage, où les choses ne sont plus complètement ce qu'elles étaient au lieu et au temps d'avant, mais sans être encore entièrement ce qu'elles sont en train de devenir... Ainsi en est-il de Berthier-surMer : le Fleuve dont l'Estuaire commence à Québec et se confirme sans retour une foie passé l'Ile d'Orléans, qui se paie le luxe narcissique de devenir Lac provisoire devant l'Anse à Berthier. Il n'en finira plus, jusqu'au détroit de Belle-Isle, d'hésiter entre toutes ses autres vocations aboutées d'Estuaire, de Golfe, de Mer et d'Océan.

$* * *$

[215] Car c'est bien ce qui, au fond, m'attache tant à ce coin de pays de Berthier-sur-Mer : une ouverture sur le monde selon la double ligne est-ouest de navigation maritime, mais aussi nord-sud des oiseaux migrateurs. « Le vaste monde », il est par la fenêtre, au bout du mur de soutènement. Au loin, les transocéaniques qui battent pavillon étranger le disent dix, vingt fois par jour. Ils sont d'Europe, de France, du Havre et donc de Paris ; ils sont d'Amérique, des Caraïbes, des États-Unis, de New York, cette métropole de notre « américanité » comme Paris 
l'est de notre « francité »; ils sont de partout sur la planète, porteurs de produits et de besoins des hommes sans parier de leurs sordides ambitions.

Cette petite patrie est comme un point d'ancrage de la grande, la planétaire, que je ne saurais assumer toute, même sur le plan des perceptions purement intellectuelles. Plus je suis accordé à ce point minuscule, plus je me sens du Monde.

Mes méditations ne sont certes pas que de cosmogonie ! C'est par mixage de tous ces éléments de paysage avec pensées latentes et volonté de détente en vacances que se produisent ces échappées vers de plus grands ailleurs... Ces enchaînements de pensée, je ne les file pas. Ils ne s'engrènent pas consciemment, ni jamais de même façon ; et seulement lorsqu'ils se produisent, ce qui n'est tout de même pas rare... Ce peut être en marchant le long de la plage pour la promenade d'exercice en entendant le clapotis des courtes vagues que le sable éponge d'un coup. Ce peut être, en levant les yeux furtivement d'une lecture d'un tout autre ordre, pour constater que ce n'est plus la même mer du coup d'oeil précédent et qui était également distrait. Ce peut être devant un feu de plage par la moiteur d'un soir de juillet, alors que tous les éléments semblent plus fluides : l'eau plus humide, l'air presque vapeur, la vapeur comme mousseline, cette mousseline que le soleil levant, demain, asséchera sans l'avoir déchirée. Ce peut être...

La mer, c'est aussi ses débris organiques de toute espèce, ses coquillages, ses anguilles éventrées dont on dit qu'elles ont de mystérieux instincts suicidaires, ses plus rares esturgeons, mais aussi les branches d'arbres, les «bois lavés » et hélas ! bouteilles, bidons et divers contenants de [216] plastique des pollueurs qui la prennent pour une poubelle.

La mer, c'est encore et en tout temps les goélands. J'entretenais à leur sujet les préjugés courants car ils sont oiseaux stupides. Ils méritent tout de même mieux que les reproductions en pacotille « artisanale » made in... Je m'empresse d'ajouter que je n'ai rien d'un bird-watcher, que je suis aussi ignare en ornithologie qu'en botanique et, à vrai dire, en toutes les sciences naturelles, ce dont je ne me loue pas, même si je serais enclin à soutenir que l'amour de la nature n'est pas corrélatif à la manie douce de la table de classification.

On a tout dit de l'extraordinaire vol plané des goélands. On leur sait moins gré d'être de grands écumeurs des plages. Ils se tiennent au loin, en tribus sur un lit de rochers et surgissent dès que les baigneurs et pique-niqueurs ont libéré la place 
après avoir sali la plage. Ils deviennent même des habitués, quoique toujours méfiants, des riverains qui leur donnent la becquée de pain rassis ou de gâteau moisi. C'est ce qui peut s'appeler s'occuper du poulailler...

Hors leur élégance de vol, ils ont tous les défauts : poltrons de la main qui les nourrit et féroces entre eux pour une miette microscopique, criailleurs et bagarreurs, aussi peu sympathiques que superbement beaux. Et trop stupides pour être calculateurs mais combien voraces! Mais leur vol... ! Il faut toujours y revenir. Cette façon qu'ils ont de planer, de faire du sur-place, de retirer leurs pattes, comme nos mécaniques volantes leur train d'atterrissage. Et quel beau spectacle lorsqu'ils font trempette comme des canards de bonne compagnie, la plus belle des escadres mouillant à l'entrée du port...

La technique d'approche pour les observer est fort simple : s'accroupir pour que l'épouvantail nourricier leur paraisse moins effrayant. Ils abolissent alors une bonne partie de la distance respectueuse d'avec les monstres que nous sommes. Si je penchais vers la misanthropie, je dirais quelles comparaisons ils me suggèrent, par leur voracité criaillante et bousculante, avec des comportements humains. Mais je ne suis pas misanthrope - non plus, du reste, que le contraire éperdument !

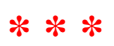

[217] Connaîtriez-vous le beau livre de l'Américain Richard Bach, Jonathan Livingston le Goéland ? Ce fut un best-seller mondial il y a une dizaine d'années, ce petit et grand livre qu'on a comparé au Petit Prince de Saint-Exupéry. C'est l'histoire de l'exclu du clan parce qu'il en refuse toute les règles, se résumant en une seule : voler pour se nourrir. Jonathan, lui, veut faire autre chose de sa vie de goéland. Il y a tant à accomplir pour se réaliser, se dépasser. Tant d'originalité insubordonnée et têtue lui vaudra une condamnation sans appel du Grand Conseil du clan des goélands. Mais, vous vous en doutez, c'est lui, et lui seul, qui gagnera à la fin (que je ne vous raconterai pas). Je transcrit seulement ce fragment de dialogue entre Jonathan et l'Ancien de l'Assemblée * :

* Jonathan Livingston, le Goéland, traduit de l'américain par Pierre Clostermann, Paris, Flammarion, 1973, pp. 26, 34. 
«... un jour Jonathan le Goéland, tu apprendras que l'irresponsabilité ne paie pas. La vie, c'est peut-être pour toi l'inconnu et l'insondable, mais nous, nous sommes mis au monde pour manger et pour demeurer vivants aussi longtemps que possible! »

Un goéland jamais ne réplique au Grand Conseil, pourtant la voix de Jonathan s'éleva :

«Irresponsabilité ? Mes frères ! s'écria-t-il, qui donc est plus responsable que le goéland qui découvre un sens plus noble à la vie et poursuit un plus haut dessein que ceux qui l'ont précédé ? Mille années durant, nous avons joué des ailes et du bec pour ramasser des têtes de poissons, mais désormais nous avons une raison de vivre : apprendre, découvrir, être libres!»

Ces quatre derniers mots vont fournir la conclusion hors-texte sur le besoin de comprendre, de l'autre côté de l'action 


\section{GÉRARD BERGERON.}

de l'autre côté de l'action.

\section{ÉPILOGUE}

\section{$\underline{\text { Retour à la table des matières }}$}

Quelques mois après l'enregistrement de ces entretiens, l'écriture-solo m'apparaît plus appropriée pour y mettre un point final par l'ajout de cet épilogue.

Ce serait d'une sincérité peu crédible que de ne pas avouer que j'ai aimé faire ces entretiens. Qui, même parmi les plus discrets d'entre les hommes et pourvu que ce soit dans telles conditions assurant confiance totale et mutuelle, n'aime pas parler de soi ? L'approche professionnelle de Jean Blouin était d'empathie à mon sujet, mais aussi sans indulgence pour le contenu des réponses de son interviewé. Avec deux brins d'astuce, il savait souvent faire rebondir le dialogue pour extorquer une réponse plus complète que ce qui venait de lui être confié.

Comme il avait exactement respecté notre convention au sujet de mon « domaine réservé » (voir le Prologue), je me devais de respecter la première règle du genre en répondant à toutes ses questions, embarrassantes ou pas ; mais plus d'une fois, je me suis senti pris par le sentiment de devoir être autant prudent que véridique dans la confidence ! Car s'il est un danger contre lequel je voulais me prémunir, c'était bien celui de sembler seulement donner dans le panneau de l'exemplarité ou de la prescription. Dire, à qui en pose la question, «dans mon cas, ça s'est posé de telle façon pour telle ou telle raison », ce n'est en rien poser cette 
façon en exemple, ni en faire la prescription à autrui. D'autant que, placé en d'autres circonstances, j'aurais pu agir autrement. Cela valait d'être redit.

Si ce «pire interviewé » de la carrière de Jean Blouin [220] (comme il m'en avait déjà fait le discutable hommage) s'est fait plus coopératif en ouvrant les vannes cette fois, c'est d'abord que le medium proposé, moins chiche d'espace, était aussi de plus grande exigence que l'article-portrait d'un magazine populaire *. Il y avait surtout une raison plus positive : quand aurais-je une autre chance de m'expliquer sur le comment les différents morceaux d'une oeuvre, d'apparence quelque peu hétéroclite, tiennent ensemble en une certaine aspiration, au moins, à l'unité ? Pourquoi n'en pas saisir l'occasion en pleine production et avant la redoutée fin de carrière?

Ces conversations prenaient un peu l'allure d'un bilan à dresser, mais je préférerais insister sur son aspect de "programme » de ce qui reste à faire. Et l'opération en devient d'autant plus compromettante qu'il faudra ensuite honorer ce dont on a avoué le projet publiquement.

En principe, c'est entendu que l'oeuvre doit se suffire à elle-même, comme disent les savants critiques. La pensée réflexive de l'auteur n'y ajoute guère, si ce n'est de l'information d'importance seconde. Tout cela est vrai surtout quand l'oeuvre est terminée, mais un peu moins, tout de même, pour la partie non encore faite de l'oeuvre à compléter. S'il est vrai, comme on le dit encore, que rien ne remplace l'oeuvre, on peut rappeler qu'il n'est à peu près personne à avoir lu toute une oeuvre, même inachevée, ni à avoir suivi l'ordre séquentiel de ses diverses publications. Si j'ai l'air de donner dans le grand genre au sujet de «l'oeuvre », c'est pour marquer autant l'enfilade chronologique des publications que leurs divers niveaux de communication. Un auteur n'est jamais connu que partiellement par un volume déterminé qu'a lu tel lecteur à tel moment ; mais il y a aussi l'ensemble des autres livres qui ont des âges inégaux et des caractères distincts comme dans toute famille. En tout cas, j'aurai trouvé plaisante cette conversationfleuve qui m'aura permis de présenter à un interlocuteur perspicace ma petite famille livresque...

Il reste tout de même une certaine dose d'exhibitionnisme dans le procédé, quelque précaution anti-narcissique ait-on pris. L'exhibitionnisme, en sa forme

* Perspectives, 29 octobre 1977, vol. 19, nº 44. 
primaire est dans [221] tout acte d'écrire pour le communiquer à autrui ; mais parler de ce qu'on a déjà écrit, voilà bien de l'exhibitionnisme au second degré ! Peuton évoquer un certain mode des 1ivres- entretiens pour y trouver une première excuse ? Ce serait d'une mince disculpation qui n'effacerait peut-être pas la double présomption du genre : qu'on a encore quelque chose à dire, qu'on le dit effectivement.

Les 1ivres- entretiens se défendraient-ils mieux par leur aspect candide et provocant. La candeur, elle est du côté de l'interviewé qui, lorsqu'il se retranche, doit avouer son retranchement ; la provocation, elle est le fait de l'interviewer qui mène le jeu, rapplique, rappelle des objections qu'il s'est déjà faites ou qu'il a déjà entendues, ne lâche pas. On ne prend pas l'initiative d'un livre pour expliquer « tout cela », qui est alentour du métier ou de l'oeuvre. Mais il aura paru naturel de répondre à des questions spécifiques dans un contexte ouvert jusqu'à la générosité, permettant une certaine élaboration bien qu'imposant, malgré tout, la contrainte de plusieurs raccourcis.

L'ensemble de ces réponses est-elle susceptible de faire crever certains malentendus ? C'est un tenace lieu commun de la critique que de discuter des incompréhensions qui se nouent entre l'auteur et son public. Ici, deux observations qu'on ne développera pas : les malentendus peuvent aller dans les deux sens, et on n'y pense guère ; les auteurs ne se plaignent pas des préjugés dits favorables, car il peut aussi s'en trouver! Je n'entends pas malentendu au sens, quelque peu grinçant, d'une divergence, inattendue ou même prévisible, de vues sur telle ou telle question fût-elle d'importance majeure dans l'actualité. La divergence peut causer de la surprise, de la déception, du ressentiment même. Le malentendu qui compte est plutôt celui d'une équivoque fondamentale portant à la fois sur la position intellectuelle générale de l'écrivant et sur le niveau propre de son discours davantage que sur son contenu brut. Donnons deux exemples.

D'une part, on veut bien accorder quelque crédit à ce que vous faites et de la confiance pour le sentiment qui vous anime, mais, d'autre part, on aimerait bien aussi vous sentir un petit peu plus engagé dans la cause à laquelle on se voue : bref, on vous souhaiterait un tantinet partisan, [222] pourvu que ce soit du «bon bord »... Mais, comme vous vous êtes imposé comme règle de salubrité de garder votre liberté entière au sein d'en engagement plus profond que l'adhésion partisane, pendant que vous décevez d'un bord vous risquez de devenir suspect de l'au- 
tre ! Encore que, ainsi qu'il a été dit lors du premier entretien, ceux dont les opinions comptent vraiment des deux bords ne s'en formalisent d'habitude pas, qui consentent même une certaine utilité sociale à cette fonction qui n'est jamais très encombrée. C'est peut-être un cas où la rareté ajoute à la valeur.

L'autre exemple est de moindre importance mais d'un peu plus de subtilité. En tant que théoricien vous pouvez construire des «modèles » ou " construits intellectuels » comme moyens de recherche en vue de la comparaison. Il s'agit toujours de pouvoir mieux juger ultérieurement de la réalité afin de voir en quels sens généraux on pourrait s'en dégager puisqu'elle est insatisfaisante, évoluant peu ou mal. Je l'ai fait consciemment en deux circonstances pour des problèmes tout à fait essentiels : un nouveau statut politico-constitutionnel du Québec nouveau et la mise en place de mécanismes moins « sauvages » de réforme constitutionnelle 22. Dans les deux cas, j'avais pris mes précautions en explicitant l'intention et la portée de l'exercice. Tel ex-ministre, capable d'agilité intellectuelle, évoquera, lors d'un colloque auquel nous participions, le premier modèle comme mon « option » personnelle entre le fédéralisme et la souveraineté-association! Ou tel critique occasionnel de journal taxera d' « angélisme » un projet de mécanismes de travail constitutionnel, pourtant conçus pour des exigences de réalisme minimal de leur fonctionnement! Les deux hommes s'étaient sentis trop dépaysés pour pouvoir suivre la convention analytique qui leur avait été proposée.

Dans l'un et l'autre cas, l'aspect « recherche » par rapport à un modèle exploratoire ou comparatif n'avait pas été pris en compte, ni même peut-être perçu. Il est vrai qu'en l'occurrence, le théoricien prenait des risques en lançant devant un large public des « construits » ou « modèles » pour interroger la réalité plutôt que pour la décrire ${ }^{23}$. Même à l'intérieur de la communauté scientifique, très peu de ses membres sont capables, dans le traitement d'une question d'un intérêt civique immédiat, de se dégager aisément d'un mode unilinéaire ou planiforme qui est le leur, soit celui de [223] l'entendement du moment ou de l'habitude de la spécialité. Des entretiens prolongés, comme ceux que je viens d'avoir avec Jean Blouin, peuvent-ils contribuer à faire éclater des malentendus de ce genre ? En tout cas, l'occasion m'aura été fournie de donner ce type de précisions sur les niveaux dis-

22 Voir l'entretien portant sur le théoricien.

23 Encore qu'il puisse y avoir un élément de prescription dans cette interrogation. D'habitude, on ne construit pas des modèles de détérioration... 
tincts et les occasions diverses du discours d'une même personne. De telles explications n'auraient pu être données ailleurs ni autrement.

Il en fut de même au sujet de la question centrale de l'indépendance dont la discussion a été la substance du premier entretien. Quand j'ai laissé tomber ce propos que l'indépendance n'aura pas lieu, ce n'était pas une profession de foi fédéraliste ou anti-séparatiste. On ne « croit » pas en ces choses comme on croit à la Sainte-Trinité ou à l'horoscope. C'est du domaine de l'examen, et d'autant plus serré, que le but en est éminemment désirable. Si l'indépendance paraissait davantage faisable, elle deviendrait encore plus désirable. Mais, même alors, il faudrait voir à quel prix ce désirable serait payé. Si l'on accédait à l'indépendance autrement que par la voie démocratique, si elle n'aboutissait pas à plus de démocratie, elle ne vaudrait pas la peine d'être vécue. C'est la grandeur du Parti québécois, et singulièrement de son chef, de maintenir une exigence intransigeante là-dessus. C'était tout au moins... Comme j'écris ces notes après les congrès péquistes de décembre 1981 et février 1982, je ne regrette pas d'avoir dit qu'il faut se méfier de tous les nationalismes, y compris du nôtre !

En ce glacial hiver 1982, la morosité du temps présent est dans toutes les bouches. L'opération, que Ian Macdonald de la Gazette avait baptisé de « Renérendum », aura produit son effet recherché de stabilisation du P.Q. Elle aura suivi de pas trop loin la propre restabilisation de son leader, capitale et plus urgente encore puisqu'il s'agit aussi de notre premier ministre. Ce parti et ce gouvernement n'en finissaient plus de mesurer leur défaite et leur isolement depuis l'évolution constitutionnelle de l'automne 1981. Demandeurs du «plus » ils étaient légitimés de se présenter comme fort crédibles défenseurs du «moins ». Ainsi en avait jugé l'électorat du 13 avril 1981, déjà tout acquis à l'idée de leur décerner un autre certificat général de bonne conduite gouvernementale. Ce parti aurait besoin d'un courage intellectuel extraordinairement lucide pour disposer [224] des illusions sur lesquelles il vit, et qui lui font perdre régulièrement référendum et sondages sur l'indépendance et gagner non moins régulièrement élections et sondages sur sa performance gouvernementale et son leadership. Sa plus grande tentation pour l'heure serait d'entretenir la mentalité de siège ; la « force des choses », plus encore économiques que constitutionnelles, est déjà en train de la lui faire passer.

L'essentiel est maintenu : l'indépendance sera acquise démocratiquement ou n'aura pas lieu. Ce n'est pas une idée exaltante, mais c'est une bonne idée pour le 
temps que durera encore la guerre civile juridique. Si l'on prenait du champ, si l'on pouvait vaincre cette déprime, même désaisonnalisée (comme on le dit pour le chômage), on pourrait se rappeler que tout aurait pu se passer tellement plus mal dans la situation de l'accession au pouvoir québécois d'un parti sécessionniste conservant toujours son projet à retardement.

Mince consolation pour le présent que la constatation que le passé n'a pas été si mal ; si seulement le futur s'annonçait en ciels moins bas... Elle est bien finie la Révolution tranquille, qui s'est prolongée en derniers reliquats qu'avait pris en charge de renouvellement le pouvoir péquiste. C'est l'ère d'une stabilisation non tranquille qui commence. Cela inclut et est surtout voyant par les compressions budgétaires. Mais c'est toute la société qui est devenue sous compression. Une société, dont tous les moteurs tournent en compression et ronronnent plus fort, va moins vite ; mais l'automobiliste sait autant que l'ingénieur que c'est aussi d'une meilleure adhésion au pavé. Le propre des difficultés économiques c'est qu'à un certain degré de leur gravité et de leur non-résolution, elles embrouillent ou annulent tout le reste. Que pèse l'indépendance du Québec, qui ne se perçoit guère que comme une idée formelle, devant l'insécurité matérielle des petites gens, des faibles, vieux et jeunes qui se trouvent dépouillés d'un horizon de clarté ?

Ce ne sont pas là propos de fatalisme, de déterminisme historique, encore moins de pessimisme. D'un certain « optimisme tragique » plutôt, selon l'expression de je ne sais quel philosophe au sujet de la vie humaine. «Tragique », [225] la vie l'est par sa fin, définitive; mais aussi «optimisme » pour tout ce qu'elle permet d'être et de faire, tant qu'elle dure, en s'accrochant à des avenirs à renouveler. La dose de « tragique » dans l'« optimisme » de notre destin collectif n'est pas à ce point contraignante qu'il faille s'embarquer dans le sacrifice, à froid, de valeurs de vie au moins aussi hautes que celles de l'indépendance. Mais ce qu'il ne faudrait pas porter aux profits et pertes, c'est cette énergie engagée dans le combat de l'indépendance. Et l'on retrouve mon inquiétude fondamentale, dont j'ai fait part à mon interlocuteur dès le début des entretiens. Elle « n'est pas que l'indépendance ait lieu ou pas, mais qu'elle ne rate pas si on s'y engage réellement ou qu'elle vienne à s'imposer vraiment un jour ». En attendant, on pourra toujours se rappeler qu'à l'échelle de la planète nous sommes parmi les peuples les plus heureux et qu'à celle de l'Occident, nous ne sommes pas parmi les plus malheureux... 
Une des questions les plus - innocemment ? - perverses de mon interviewer a été celle portant sur l'hypothétique crédit qu'aurait le théoricien à ce titre quand il se mue en critique journalistique des conjonctures concrètes. Je renvoie à ma réponse embarrassée du troisième entretien. La réputation scientifique s'édifie par la qualité des travaux de recherche que reconnaissent les pairs au sein de la spécialité. Mais il est aussi un autre type de reconnaissance qu'à partir de critères autres 24 décerne «la presse intellectuelle ». C'est singulièrement le cas lorsque le politologue descend dans l'arène publique. Qu'il le fasse en «spectateur engagé », selon la formule de Raymond Aron, ou sous l'impératif d'un « engagement dégagé », selon celle de Jacques Juilliard, ces nuances importent peu. Le marché ou virtuel marketing d'un écrivain est incomparablement plus vaste par ses interventions journalistiques que par le tirage de ses livres. Un texte mis en valeur dans un journal comme Le Devoir ou une participation à la télévision à un moment de chaude actualité valent d'un coup à l'intervenant un public plus large que celui que n'obtiendront jamais ses oeuvres complètes. En outre, avantage dubitatif mais spécifique à la télé : « une tête » identifiera dorénavant le nom de celui qui la porte et qui n'était jusque là qu'une signature.

24 Selon Raymond Boudon, « dans ce cas, l'intellectuel 'saisit' non pas le milieu universitaire, mais le milieu qu'on peut appeler 'intellectuel'. Il compose alors son produit en des termes tels qu'il puisse obtenir un écho rapide dans la presse intellectuelle... Le recours à ce système de gratification suppose que le produit traite d'un thème d'intérêt actuel et général, que les analyses qu'il présente soient suffisamment dépouillées de technicité tout en éveillant parfois des sentiments de profondeur ; que les conclusions de l'analyse puissent être réunies à l'aide de quelques propositions simples sans que l'argumentation soit dépourvue d' 'épaisseur'. Le recours à ce système de gratification présente naturellement des avantages. Il libère le producteur des contraintes de l'éthique scientiste. Il peut fournir des gratifications plus rapides et plus tangibles. Il permet de toucher d'un coup des marchés divers. Il présente aussi des désavantages : la notoriété qu'il est capable de conférer se révèle parfois peu durable » (Effet pervers et ordre social, Paris, PUF, 1977, p. 222-3). 
[226] Depuis l'enregistrement des entretiens précédents, j'ai pris connaissance du livre de François Bourricaud, Le bricolage intellectuel ${ }^{25}$, essai sociologique d'une courageuse lucidité. Le terme ne doit pas s'entendre qu'à l'ironie et qu' « en mauvaise part » 26 . Pourquoi, «nous sommes tous des bricoleurs » ? - Du fait que « les intellectuels sont les spécialistes du débat idéologique, et comme le débat idéologique, compte tenu de la nature des objets qui y sont traités, ne se rapproche que de très loin d'une véritable discussion scientifique, les intellectuels sont condamnés au bricolage. Cette formule ne se comprend bien que si l'on entend l'idéologie comme la culture, altérée ou rendue contentieuse par des spécialistes de l'argumentation, qui se proposent d'en faire ressortir les points forts aux yeux d'un public plus ou moins étendu » 27 . Le public est plus étendu lorsqu'il est rejoint par les média ; mais les contraintes de leur technicité même, avec un paradoxal effet d'amplification, forcent non seulement au bricolage, mais à toujours plus de bricolage. De là pour un public mondain, largement ouvert et sans défense critique, à identifier l'activité intellectuelle au bricolage de parade...

Mon propos s'applique dans le Québec d'aujourd'hui à une certaine pratique mondaine des sciences sociales, à l'exclusion donc de la pratique des sciences de la nature qui, comme "activité scientifique », sont plus sérieuses et compromettantes. Dans des milieux culturels plus vastes et aux centres plus éparpillés comme au Canada anglais et, surtout, aux États-Unis, où les gratifications symboliques ont plus de netteté « scientifique », le tableau serait plus complexe du fait, encore, de l'existence d'une critique non moins exigeante que la production des

25 Portant comme sous-titre : Essai sur les intellectuels et les passions démocratiques, Paris, PUF, 1980.

26 «Il ne faut pas le prendre seulement en mauvaise part. Il vise à dénoncer l'imposture de ceux qui cherchent par innocence ou par perversité à nous faire prendre des vessies pour des lanternes, les crimes totalitaires pour la 'réalisation concrète de la liberté'. Mais sous son apparence dérisoire, il désigne aussi l'activité besogneuse de ceux qui, même s'ils n'ont pas la maîtrise des problèmes qu'ils évoquent, en cherchent modestement la solution. À ce titre, nous sommes tous des bricoleurs. Du bricolage des idéologues sortent parfois des bribes de connaissance. Les idéologues qui sont maîtres d'erreur quand ils nous donnent leurs élucubrations pour de la science peuvent aussi contribuer à son progrès. Non seulement ils dénoncent les scandales, mais ils débusquent les paradoxes. C'est par la résolution méthodique de paradoxes que nous nous engageons dans la voie du travail scientifique » (Ibid., p. 11.).

27 Ibid., p. 66. 
travaux. Au Québec, faiblesse du nombre ou jeunesse de notre maturité intellectuelle, la critique ne suit pas toujours pour de nécessaires discriminations.

Retour au bricolage, certes il y a bricoleur et bricoleur, comme il y a ingéneur et ingénieur, architecte et architecte ! Voué qu'il est à une certaine forme de bricolage intellectuel, au moins en partie justifiable par l'accomplissement d'un devoir civique, le bricoleur doit s'en rendre compte, au moins de temps en temps. Et là conscience qu'il en a, si elle ne suffit pas à lui interdire complètement de bricoler, doit au moins le prémunir contre le danger de proclamer que [227] c'est autre chose. Quant à l'activité scientifique et de rigueur intellectuelle, elle n'est toujours qu'une pénible aspiration vers...

En attendant, il faut continuer à vivre. À vivre en société. Dans une société qui est « politique » de quelque angle qu'on la considère, puisque ses problèmes généraux, qui ne se résolvent jamais, se posent toujours en termes du politique, ce qui est de beaucoup plus grande nécessité que les verbiages et les bousculades de la politique. 
[228]

GÉRARD BERGERON.

de l'autre côté de l'action.

NOTICE BIOGRAPHIQUE

Retour à la table des matières

Gérard Bergeron est né le 31 janvier 1922 à Charny, près de Québec. Études secondaires au Collège de Lévis (1936-1944). Études universitaires aux universités Laval, Columbia, de Genève et de Paris (1944-1950).

Professeur à la Faculté des sciences sociales de l'université Laval (département de sociologie : 1950-1954 et au département de science politique depuis 1954). Professeur titulaire de Théorie de l'État (1962). Professeur à l'École nationale d'Administration publique (depuis juin 1981). 
[229]

\author{
GÉRARD BERGERON. \\ de l'autre côté de l'action. \\ BIBLIOGRAPHIE
}

Théorie politique

Retour à la table des matières

Fonctionnement de l'État, Paris, Armand Colin, et Québec, Les Presses de l'Université Laval, 1965 (avec une préface de Raymond Aron) ; 660 pages.

La guerre froide inachevée, Montréal, Les Presses de l'Université de Montréal, 1971 (avec une préface de John Holmes) ; 315 pages.

La gouverne politique, Paris-La Haye, Mouton et Québec, les Presses de l'Université Laval, 1977 ; 264 pages.

Mécanismes d'élaboration d'une constitution nouvelle, (en coll. avec Gérald Beaudoin, Edward McWhinney et Edmond Orban), Ottawa, Les Presses de l'Université d'Ottawa, 1981 ; 146 pages. 


\section{Essais historiques}

Le Canada français après deux siècles de patience, Paris, Le Seuil, 1967 ; 281 pages.

Incertitudes d'un certain pays, Québec, Les Presses de l'Université Laval, $1979 ; 270$ pages.

L'État du Québec en devenir (en collaboration avec Réjean Pelletier et al.), Montréal, Boréal Express, 1980 ; 413 pages.

\section{Études de conjonctures}

Du duplessisme au johnsonisme, Montréal, Éditions Parti Pris, 1967 ; 470 pages.

Ne bougez plus! (portraits de politiciens), Montréal, Éditions du Jour, 1968, 224 pages.

Du duplessisme à Trudeau et Bourassa, Montréal, Éditions Parti Pris, 1971 ; 631 pages.

L'indépendance : oui, mais.., Montréal, Éditions Quinze, 1977 (avec une préface de Pierre Turgeon) ; 198 pages.

Ce jour-là... le référendum, Montréal, Éditions Quinze, 1978 ; 256 pages.

Syndrome québécois et mai canadien, Québec, Les Presses de l'Université Laval, 1981 (avec une préface de Louis Duclos) ; 298 pages.

\section{Fin du texte}

